\title{
TABLES OF THEORETICAL ZEEMAN EFFECTS
}

\author{
By C. C. Kiess and W. F. Meggers
}

\begin{abstract}
The splitting of spectrum lines into components, when the source is in a magnetic field, Zeeman effect, furnishes theoretically an absolute identification of the terms involved in the production of the lines. Based on the theoretical work of Landé the Zeeman effects of various term combinations have been computed, and these are now presented in tabular form. The tables give results for terms from $\mathrm{S}$ to I in the doublet, quartet, sextet, and octet systems; in the triplet, quintet, and septet systems; and in the doublet-quartet, quartet-sextet, sextetoctet, singlet-triplet, triplet-quintet, and quintet-septet intersystems.
\end{abstract}

\section{CONTENTS}

I. Introduction

II. Tables_... 648

\section{INTRODUCTION}

The classification of the lines of a spectrum as differences between terms of various types has made rapid progress in recent years. Especially is this true of the complex spectra of the elements occupying the columns on the right of the periodic table. This work has been aided by several kinds of physical phenomena which reveal the existence of a relationship between the members of a group of neighboring spectrum lines. Among these aids may be mentioned the temperature classification of King, the reversibility of lines under various conditions, the red-ward shift of lines with pressure, and the splitting of lines in electric (Stark effect) and magnetic fields (Zeeman effect).

Of these aids the Zeeman effect, observed in weak fields, furnishes theoretically an absolute identification of the term combination represented by a spectrum line. The theory of the Zeeman effect derived by Lorentz from the classical mechanics accounted only for the so-called normal triplets. The explanation of the "anomalous" Zeeman effect, which is characteristic of the majority of lines, was possible only after the development of modern theories of spectra based on the quantum theory of atomic structure.

The idea that the splitting of a line in the magnetic field was the result of a splitting of the terms which combine to produce the line first originated with Van Lohuizen. ${ }^{1}$ The subsequent development of

1 Proc. Acad. Amsterdam, 22, p. 190; 1919.

$9414^{\circ}-28-1$ 
this idea by Sommerfeld, ${ }^{2}$ and others, culminated in the work of Lande, ${ }^{3}$ who, guided largely by the high precision observations of Back, was able to give a formula for the splitting of a term in the magnetic field. This formula expresses a relation between the quantum numbers necessary to completely specify a spectral term.

The terms of a spectrum correspond to the various energy states of the emitting atom and their types may be calculated, as shown by Hund, ${ }^{4}$ from the different configurations which the valence electrons of the atom assume when it is excited. In addition to the total quantum number $n$, which tells which shell it is in, each electron is specified by the quantum numbers $l_{1}(=0,1,2,3, \ldots$, for $s, p, d$, $f, \ldots$, electrons, respectively), and $s_{1}(= \pm 1 / 2)$, which state the number of units of quantized angular momenta associated with their orbital revolutions and axial rotations. Any term ${ }^{\mathrm{r}} \mathrm{T}_{\mathrm{j}}$ represents quantitatively one of the resultants obtained by adding together vectorially the orbital and axial angular momenta of the electrons composing a particular configuration. Thus, $l=\Sigma l_{1}=0,1,2,3$, $\ldots$ for S, P, D, F, . . terms, and $s=\Sigma s_{1}=0,1 / 2,1,3 / 2,2, \ldots$. for singlets, doublets, triplets, quartets, quintets, etc. The inner quantum numbers, $j$, which represent mechanically the resultant angular momentum of the ätom, are given by the relations $j_{\max }=$ $l+s$, and $j_{\min }=l-s$; and the multiplicity by $r=2 s+1$.

When the atom is in a magnetic field it behaves like a spinning top subject to external force moment; that is, its axis of resultant angular momentum, the axis of $j$, describes a precessional cone (Larmor precession) about the direction of the field. Since the motion is quantized, only those angles of the cone are permitted which correspond to integral projections of $j$ on the field in the case of odd multiplicities, and half-integral projections for even multiplicities. The values of these projections are designated by the magnetic quantum number $m$, and for any value of $j$, there will be $2 j+1$ values of $m$, proceeding by steps of one unit from $+j$ to $-j$. In other words, the effect of the field is to add equal increments of energy to the atom, the number of such increments being restricted to the number of orientations which the atom, in a particular state, can assume with respect to the field. This is equivalent to splitting up the term corresponding to the energy state into a group of equidistant components, each of which corresponds to an increment of added energy. The amount by which the components of a split term are separated from each other is given by the relation

$$
\Delta \nu=\frac{\mu}{j} \frac{H}{h} m
$$

2 Annalen der Physik, 63, p. 221; 1920.

${ }^{3}$ Zeitschrift für Physik, 5, p. 231, 1921; 15, p. 189, 1923.

- Linienspektren und periodisches System der Elemente, Berlin, Julius Springer; 1927. 
in which $\mu$ is the magnetic moment of the atom, $H$ is the strength of the magnetic field, $h$ is Planck's constant, and $m$ and $j$ have the meanings defined above. Expressed in terms of the normal Zeeman triplet, this reduces to

$$
\Delta \nu=\frac{\mu}{j} m=g m
$$

The ratio of the magnetic to the mechanical moment of the atom $\mu / j=g$ is Landés splitting factor. By a process of induction based on the $g$ values of known terms Landé was able to set up a formula expressing $g$ as a function of the quantum numbers which specify a spectral term. This formula has been found to hold, generally, for terms of all types and multiplicities, and expressed in notation of current usage (Sommerfeld's ${ }^{5}$ ) is

$$
g=1+\frac{j(j+1)+s(s+1)-l(l+1)}{2 j(j+1)}
$$

As an illustrative problem, let it be required to find the $g$ value of the term ${ }^{5} \mathrm{~F}_{4}$. Here $j=4, s=\frac{r-1}{2}=2$ and $l=3$. Substitution of these values in the formula gives $g=1+\frac{14}{40}=\frac{54}{40}=\frac{27}{20}$. By such a procedure tables of $g$ values have been constructed which may be found in any one of a number of works. ${ }^{6}$

When the atom in the magnetic field radiates light the components of one magnetically resolved term group combine with those of another such group in accordance with the rules governing the combination. Each component term is specified by a particular value of the magnetic quantum number $m$ and only those combinations occur for which the change in $m$ is \pm 1 or 0 , with the requirement that the combination of two terms for each of which $m=0$ is forbidden. If two terms combine for which $\Delta m= \pm 1$ the radiated light is circularly polarized and the components of the Zeeman pattern perpendicular to the field are observed. If $\Delta m=0$ the radiated light is plane polarized and the parallel components are observed. As illustrations, let it be required to calculate the theoretical Zeeman patterns of the term combinations $(a),{ }^{5} \mathrm{~F}_{3}-{ }^{5} \mathrm{G}_{4},(b),{ }^{4} \mathrm{D}_{3}-{ }^{4} \mathrm{~F}_{3}$.

3 Three lectures on atomic physics. Lecture II, London. Methuen \& Co.; 1926.

- Back u. Lande, Zeemaneffekt und Multiplettstruktur der Spektrallinien, p. 42, Berlin, Julius Springer: 1925. Sommerfeld, Atombau. 4th ed., p. 623, Braunschweig, Vieweg \& Sohn: 1924. Zeeman and De Bruin, Handbuch der Physikalischen Optik, 2, p. 638: 1927. 
(a) The $g$ values for ${ }^{5} \mathrm{~F}_{3}$ and ${ }^{5} \mathrm{G}_{4}$ are $\frac{15}{12}$ and $\frac{23}{20}$, or $\frac{75}{60}$ and $\frac{69}{60}$. For ${ }^{5} \mathrm{~F}_{3}$ the magnetic quantum number $m$ runs from -3 to +3 , and for ${ }^{5} \mathrm{G}_{4}$ from -4 to +4 . We have the following values of $m g$ for each term:

\begin{tabular}{|c|c|c|c|c|c|c|c|c|c|}
\hline$m$ & -4 & -3 & -2 & -1 & 0 & +1 & +2 & +3 & +4 \\
\hline${ }^{5} \mathrm{~F}_{3}$ & & $-\frac{225}{60}$ & $-\frac{150}{60}$ & $-\frac{75}{60}$ & $\frac{0}{60}$ & $+\frac{75}{60}$ & $+\frac{150}{60}$ & $+\frac{225}{60}$ & \\
\hline${ }^{5} \mathrm{G}_{4}$ & $-\frac{276}{60}$ & $-\frac{207}{60}$ & $-\frac{138}{60}$ & $-\frac{69}{60}$ & $\frac{0}{60}$ & $+\frac{69}{60}$ & $+\frac{138}{60}$ & $+\frac{207}{60}$ & $+\frac{276}{60}$ \\
\hline
\end{tabular}

By subtracting the fractions of the lower row from those above, first vertically and then diagonally, we get, respectively, the components of the Zeeman pattern for which $\Delta m=0$ and $\Delta m= \pm 1$. The collected results are expressed as

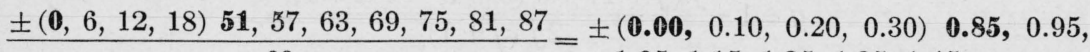
$60 \quad 1.05,1.15,1.25,1.35,1.45$

the parallel components being the ones inclosed in ( ), the perpendicular components following.

(b) The $g$ values are $\frac{48}{35}$ and $\frac{36}{35}$ for ${ }^{4} \mathrm{D}_{3}$ and ${ }^{4} \mathrm{~F}_{3}$.

Note.-The inner quantum numbers of terms of even multiplicity are halfintegers. For convenience in printing the integer greater by $1 / 2$ than the proper inner quantum number is used throughout this paper.

The labor of computation is diminished by using the decimal equivalents of the $g$ 's, especially if a table of products or a slide rule are available. Thus, since

$$
\frac{48}{35}=1.371 \text { and } \frac{36}{35}=1.029
$$

\begin{tabular}{|l|rrrrrr|}
\hline$m$ & $-\frac{5}{2}$ & $-\frac{3}{2}$ & $-\frac{1}{2}$ & $+\frac{1}{2}$ & $+\frac{3}{2}$ & $+\frac{5}{2}$ \\
\hline${ }^{4} \mathrm{D}_{3}$ & -3.43 & -2.06 & -0.69 & +0.69 & +2.06 & +3.43 \\
${ }^{4} \mathrm{~F}_{3}$ & -2.57 & -1.54 & -.52 & +.52 & +1.54 & +2.57 \\
\hline
\end{tabular}

Whence, taking vertical differences for parallel components, and diagonal differences for perpendicular components, we obtain

$$
\pm(0.17,0.51,0.86) 0.51,0.86,1.20,1.54,1.89
$$


The components printed in heavy type are the most intense. These are the ones, according to Landé, which correspond to the maximum angle between the axis of $j$ and the direction of the field for parallel components, and to the minimum angle for perpendicular components.

In determining these, the following practical rules hold. In case the $j$ 's of the combining terms are not equal, problem (a) above, the vertical differences in the middle of the scheme and the diagonal differences at the ends give, respectively, the strongest $p$ and $n$ components. In case the $j$ 's are equal, problem $(b)$, the vertical differences at the end of the scheme and the diagonal differences at the center give, respectively, the strongest $p$ and $n$ components, with the added requirement that for terms of odd multiplicity the $p$ components corresponding to the transition $m=0$ to $m=0$ are forbidden; that is, their intensity is zero.

Workers, engaged in classifying spectra, find it convenient to have at hand tables of Zeeman effects for the various term combinations. During the past five years such tables have been calculated from time to time at the Bureau of Standards, according to the requirements of the spectrum under investigation. These tables are now extensive enough to cover all possible term combinations which are likely to occur in the spectra of elements other than the rare earths. For certain elements there is some evidence that $g$ values differing from those given by the formula above will be required, but the exact law governing such anomalous values is not yet known. For these reasons, it is believed that these tables for the normal $g$ values will be useful because of their completeness, and their publication will save the unnecessary time and labor of duplication on the part of others.

The tables for each series system are preceded by a table of $g$ values for that system which differ from those referred to above only in that the fractions in each vertical column have all been converted to the same common denominator. When this is done, an inspection of the tables shows simple relations between the numerators and denominators of the g's not only in the vertical columns, but also in the horizontal and diagonal rows, which permit writing down additional $g$ values without recourse to the above formula.

The art of observation has not sufficed to separate Zeeman patterns whose components differ by less than $1 / 5 a$, for sources in air, or by less than $\frac{1}{10} a$ for vacuum sources, $a$ being the normal triplet separation. The result is that many observed patterns represent the blending of overlapping images, so that in order to interpret them, the theoretical patterns of the following tables must be somewhat modified. Such a procedure was adopted by Russell ${ }^{7}$ in inter-

7 Astrophysical J. 66, p. 307; 1927. 
preting observed Zeeman effects of Ti. Weighting the lines according to their theoretical intensities, as done by Hönl, ${ }^{8}$ he found that by placing the center of an unresolved group at one-fourth the way from the strongest to the weakest component, modified theoretical patterns could be derived which would satisfactorily fit those observed.

Although it is the purpose of the following tables to furnish an interpretation of any observed Zeeman effect, yet it sometimes happens that a pattern is encountered which deviates from the theoretical patterns to an extent that makes its identification uncertain. In such a case, it is often helpful to determine the $g$ values of the combining terms from the observed pattern and thereby arrive at an identification of the terms. This procedure has been well illustrated by Back; ${ }^{9}$ it involves the solution of two simple linear equations with two unknown quantities. Let $g_{\mathrm{x}}$ and $g_{\mathrm{y}}$ be the $g$ 's of the unknown terms with inner quantum numbers $j_{\mathrm{x}}$ and $j_{\mathrm{y}}$, and let $m_{\mathrm{x}}$ and $m_{\mathrm{y}}=m_{\mathrm{x}} \pm 1$ be the magnetic quantum numbers corresponding to the terms which give the $n$ components of maximum intensity. Further, let $e$ represent the mean separation of the components of the observed pattern, and let $2 f$ be the separation of the $n$ components of maximum intensity. Then, in case $j_{\mathrm{x}}$ and $j_{\mathrm{y}}$ are not equal, the two equations are

$$
\begin{gathered}
g_{\mathrm{x}}-g_{\mathrm{y}}= \pm e \\
m_{\mathrm{x}} g_{\mathrm{x}}-m_{\mathrm{y}} g_{\mathrm{y}}= \pm f
\end{gathered}
$$

In case $j_{\mathrm{x}}$ equals $j_{\mathrm{y}}$ then, owing to the fact that the strong $n$ components are given by the diagonal differences at the center of the pattern, the values of the magnetic quantum numbers are $m_{\mathrm{y}}= \pm 1$ for $m_{\mathrm{x}}=0$, or $m_{\mathrm{y}}=0$ for $m_{\mathrm{x}}= \pm 1$, for terms of odd multiplicity; and $m_{\mathrm{y}}= \pm 1 / 2$ for $m_{\mathrm{x}}=\mp 1 / 2$ for terms of even multiplicity. In the case of odd multiplicity terms there will be, in general, two $n$ components of the same maximum intensity and accordingly two values of $2 f$. The equations to be solved are therefore odd multiplicity

even multiplicity

$$
\begin{gathered}
g_{\mathrm{x}}-g_{\mathrm{y}}= \pm e= \pm\left(f_{2}-f_{1}\right) \\
g_{\mathrm{x}}=f_{1} \\
g_{\mathrm{y}}=f_{2}
\end{gathered}
$$

$$
g_{\mathrm{x}}-g_{\mathrm{y}}= \pm e
$$$$
1 / 2 g_{\mathrm{x}}-1 / 2 g_{\mathrm{y}}= \pm f
$$ 
In illustration of the above, let it be required to find the $g$ values of the terms which combine to produce the $\mathrm{Cb}$ are lines 4123.86 and 4163.64, for which Jack ${ }^{10}$ observed the Zeeman effects

(a) $(0.32,0.90) 0.4 \%, 1.02,1.58,2.18$

(b) $(-, 1.18) 0.66,1.46,2.24$

The terms are of even multiplicity, $(a)$ representing a combination for which $j_{\mathrm{x}} \neq j_{\mathrm{y}}$ and $(b)$ a combination for which $j_{\mathrm{x}}=j_{\mathrm{y}}$ For $(a)$, we have

$$
\begin{aligned}
e & = \pm 0.57 ; f= \pm 0.47 \\
\therefore g_{x}-g_{y} & = \pm 0.57 \\
\frac{3}{2} g_{x}-\frac{5}{2} g_{y} & =\mp 0.47
\end{aligned}
$$

whence

$$
g_{\mathrm{x}}=1.90 \text { corresponding to } \frac{28}{15}\left({ }^{6} \mathrm{D}_{2}\right)
$$

and

For $(b)$ we have

$$
g_{y}=1.33 \text { corresponding to } \frac{4}{3} \text { or } \frac{46}{35}\left({ }^{e} \mathrm{~F}_{3}\right)
$$

$$
\begin{aligned}
e & = \pm 0.79, f= \pm 1.46 \\
\therefore g_{x}-g_{y} & = \pm 0.79 \\
1 / 2 g_{x}+1 / 2 g_{y} & = \pm 1.46
\end{aligned}
$$

whence

$$
g_{\mathrm{x}}=1.86 \text { corresponding to } \frac{28}{15}\left({ }^{6} \mathrm{D}_{2}\right)
$$

and

$$
g_{\mathrm{y}}=1.07 \text { corresponding to } \frac{16}{15}\left({ }^{6} \mathrm{~F}_{2}\right)
$$

To facilitate the identification of the terms corresponding to computed $g$ values, as in the foregoing problems, Tables 14 and 15 have been compiled.

In conclusion we wish to acknowledge our indebtedness to Prof. H. N. Russell, of Princeton University, who kindly checked our tables with those computed by him.

${ }^{10}$ Proc. Roy. Irish Acad., 30A, p. 42; 1912. 


\section{TABLES}

TABLE 1.-Theoretical Zeeman effects (doublet system)

[Landé $g$ values]

\begin{tabular}{|c|c|c|c|c|c|c|c|c|c|c|c|c|c|c|}
\hline$l^{j}$ & 1 & 2 & 3 & 4 & 5 & 6 & 7 & 1 & 2 & 3 & 4 & 5 & 6 & 7 \\
\hline $\mathrm{s}$ & $\frac{6}{3}$ & & & & & & & 2. 000 & & & & & & \\
\hline $\mathrm{P}$ & $\frac{2}{3}$ & $\frac{20}{15}$ & & & & & & 0.667 & 1. 333 & & & & & \\
\hline D & & $\frac{12}{18}$ & $\frac{4}{36}$ & & & & & & 0.800 & 1. 200 & & & & \\
\hline F & & & $\frac{30}{38}$ & $\frac{72}{6} \frac{2}{3}$ & & & & & & 0.857 & 1. 143 & & & \\
\hline G & & s. & & $\frac{86}{83}$ & 1100 & & & & & & 0.889 & 1.111 & & \\
\hline $\mathrm{H}$ & & & & & $\frac{90}{60}$ & $\frac{158}{143^{3}}$ & & & & & & 0.909 & 1. 091 & \\
\hline I & & & & & & $\frac{152}{143}$ & $\frac{210}{10}$ & & & & & & 0.923 & 1. 077 \\
\hline
\end{tabular}

${ }^{2} \mathrm{~S}_{1}-2 \mathrm{~S}_{1}{ }_{1}(0.00), 2.00$.

${ }^{2} \mathrm{~S}_{1}-2 \mathrm{P}_{1}(0.67), 1.33$.

${ }^{2} \mathrm{~S}_{1}-2 \mathrm{P}_{2}(0.33), 1.00,1.67$.

${ }^{2} \mathrm{~S}_{1}-2 \mathrm{D}_{3}^{\prime}(0.60), 0.20,1.40$.

${ }^{2} \mathrm{P}_{1}-2 \mathrm{P}^{\prime}{ }_{1}(0.00), 0.67$.

. $\left.{ }^{2} \mathrm{P}_{1}-2 \mathrm{P}_{2}^{\prime}\right\}$

$\left.{ }^{2} \mathrm{P}_{2}-2 \mathrm{P}_{1}^{\prime}\right\}$ (0.33), 1.00, 1.6\%.

${ }^{2} \mathrm{P}_{2}-2 \mathrm{P}_{2}^{\prime}(0.00), 1.33$.

${ }^{2} \mathrm{P}_{1}-2 \mathrm{D}_{2}(0.07), 0.73,0.87$.

${ }^{2} \mathrm{P}_{2}{ }^{-2} \mathrm{D}_{2}(0.27,0.80), 0.53,1.07,1.60$.

${ }^{2} \mathrm{P}_{2}-{ }^{2} \mathrm{D}_{3}(0.07,0.20), 1.00,1.13,1.27,1.40$.

${ }^{2} \mathrm{P}_{2}-2 \mathrm{~F}_{3}^{\prime}(0.21,0.71), 0.14,0.62,1.09,1.57$.

${ }^{2} \mathrm{D}_{2}-{ }^{2} \mathrm{D}_{2}^{\prime}(0.00), 0.80$.

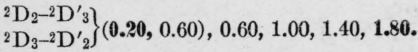

${ }^{2} \mathrm{D}_{3}-{ }^{2} \mathrm{D}_{3}^{\prime}{ }_{3}(0.00), 1.20$.

${ }^{2} \mathrm{D}_{2}{ }^{-2} \mathrm{~F}_{3}(\mathbf{0 . 0 3}, 0.09), 0.77,0.83,0.89,0.94$.

${ }^{2} \mathrm{D}_{3}-{ }^{2} \mathrm{~F}_{3}(0.17,0.51,0.86), 0.34,0.69,1.03,1.37,1.71$.

${ }^{2} \mathrm{D}_{3}-2 \mathrm{~F}_{4}(0.03,0.09,0.14), 1.00,1.06,1.11,1.17,1.23,1.28$.

${ }^{2} \mathrm{D}_{3}-{ }^{2} \mathrm{G}_{4}^{\prime}(0.16,0.47,0.78), 0.11,0.42,0.73,1.04,1.35,1.67$.

${ }^{2} \mathrm{~F}_{3}-2 \mathrm{~F}^{\prime}{ }_{3}(0.00), 0.86$.

$\left.{ }^{2} \mathrm{~F}_{3}-{ }^{2} \mathrm{~F}_{4}^{\prime} \mathrm{F}_{4}-2 \mathrm{~F}_{3}^{\prime}{ }_{3}\right\}(0.14,0.43,0.71), 0.43,0.71,1.00,1.28,1.57,1.86$.

${ }^{2} \mathrm{~F}_{4}-{ }^{2} \mathrm{~F}_{4}^{\prime}(0.00), 1.14$

${ }^{2} \mathrm{~F}_{3}-{ }^{2} \mathrm{G}_{4}(\mathbf{0 . 0 2}, 0.05,0.08), 0.81,0.84,0.87,0.90,0.94,0.97$.

${ }^{2} \mathrm{~F}_{4}-{ }^{2} \mathrm{G}_{4}(0.13,0.38,0.64,0.89), 0.25,0.51,0.76,1.02,1.27,1.52,1.78$.

${ }^{2} \mathrm{~F}_{4}{ }^{-2} \mathrm{G}_{5}(0.02,0.05,0.08,0.11), 1.00,1.03,1.06,1.10,1.13,1.16,1.19,1.22$.

${ }^{2} \mathrm{~F}_{4}{ }^{2} \mathrm{H}^{\prime}{ }_{5}(0.12,0.35,0.58,0.82), \mathbf{0 . 0 9}, 0.32,0.56,0.79,1.03,1.26,1.49,1.73$.

${ }^{2} \mathrm{G}_{4}-{ }^{2} \mathrm{G}_{4}^{\prime}(0.00), 0.89$.

$\left.{ }^{2} \mathrm{G}_{4}-{ }^{2} \mathrm{G}^{\prime}{ }_{5}\right\}$

$\left.{ }^{2} \mathrm{G}_{5}-\mathrm{C}^{2} \mathrm{G}_{4}\right\}(0.11,0.33,0.56,0.78), 0.33,0.56,0.78,1.00,1.22,1.44,1.67,1.89$.

${ }^{2} \mathrm{G}_{5}-{ }^{2} \mathrm{G}_{5}{ }_{5}(0.00), 1.11$.

${ }^{2} \mathrm{G}_{4}-{ }^{2} \mathrm{H}_{5}(0.01,0.03,0.05,0.07), 0.84,0.86,0.88,0.90,0.92,0.94,0.96,0.98$.

${ }^{2} \mathrm{G}_{5}-{ }^{-2} \mathrm{H}_{5}(0.10,0.30,0.50,0.71,0.91), 0.20,0.40,0.61,0.81,1.01,1.21,1.41,1.62,1.82$.

${ }^{2} \mathrm{G}_{5}-{ }^{2} \mathrm{H}_{6}(0.01,0.03,0.05,0.07,0.09), 1.00,1.02,1.04,1.06,1.08,1.10,1.12,1.14,1.16,1.18$

$\left\{G_{5}-2 I_{6}^{\prime}(0.09,0.28,0.47,0.66,0.85), 0.08,0.26,0.45,0.64,0.83,1.02,1.21,1.39,1.58,1.77\right.$. 
TABLE 1.-Theoretical Zeeman effects (doublet system)-Continued

${ }^{2} \mathrm{H}_{5}-2 \mathrm{H}^{\prime}{ }_{5}(0.00), 0.91$.

$\left.{ }^{2} \mathrm{H}_{5}-{ }^{2} \mathrm{H}_{6}^{\prime}\right)$

$\left.{ }^{2} \mathrm{H}_{6}{ }^{2} \mathrm{H}_{5}{ }_{5}\right\}(0.98,0.27,0.45,0.64,0.82), 0.27,0.45,0.64,0.82,1.00,1.18,1.36,1.54,1.73,1.91$.

${ }^{2} \mathrm{H}_{6}{ }^{-2} \mathrm{H}_{6}^{\prime}(0.00), 1.09$.

${ }^{2} \mathrm{H}_{5}{ }^{-2} \mathrm{I}_{6}(0.81,0.02,0.03,0.05,0.06), 0.86,0.88,0.89,0.90,0.92,0.93,0.94,0.96,0.97,0.88$.

${ }^{2} \mathrm{H}_{6}{ }^{-2} \mathrm{I}_{6}(0.08,0.25,0.42,0.59,0.76,0.92), 0.17,0.34,0.50,0.67,0.84,1.01,1.17,1.34,1.51,1.68,1.85$.

${ }^{2} \mathrm{H}_{6}{ }^{-2} \mathrm{I}_{7}(0.01,0.02,0.03,0.05,0.06,0.08), 1.00,1.01,1.03,1.04,1.05,1.06,1.08,1.10,1.11,1.12,1.14,1.15$.

${ }^{2} \mathrm{I}_{6}-2 \mathrm{I}_{6}{ }_{6}(0.00), 0.92$.

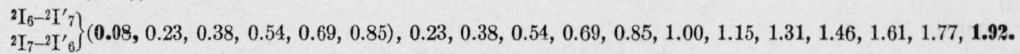

${ }^{2} \mathrm{I}_{7}-{ }^{2} \mathrm{I}_{7}^{\prime}(0.00), 1.08$.

TABLE 2.-Theoretical Zeeman effects (quartet system)

[Landé $g$ values]

\begin{tabular}{|c|c|c|c|c|c|c|c|c|c|c|c|c|c|c|c|c|}
\hline$i \backslash j$ & 1 & 2 & 3 & 4 & 5 & 6 & 7 & 8 & 1 & 2 & 3 & 4 & 5 & 6 & 7 & 8 \\
\hline $\mathrm{s}$ & & $\frac{30}{15}$ & & & & & & & & 2.000 & & & & & & \\
\hline $\mathrm{P}$ & $\frac{8}{3}$ & $\frac{26}{1} \frac{6}{6}$ & $\frac{56}{36}$ & & & & te & & 2. 667 & 1.733 & 1. 600 & & & & & \\
\hline D & $\frac{0}{3}$ & $\frac{18}{16}$ & $\frac{48}{36}$ & $\frac{90}{63}$ & & & & & 0.000 & 1. 200 & 1. 371 & 1. 429 & & & & \\
\hline $\mathrm{F}$ & & $\frac{6}{18}$ & $\frac{36}{38}$ & $\frac{7}{63}$ & $\frac{132}{98}$ & & & & & 0.400 & 1. 029 & 1. 238 & 1. 333 & & & \\
\hline G & & & $\frac{20}{36}$ & $\frac{6 \frac{2}{6}}{63}$ & $\frac{116}{98}$ & $\frac{18 \frac{2}{14}}{3}$ & & & & & 0.571 & 0.984 & 1. 172 & 1. 273 & & \\
\hline $\mathrm{H}$ & & & & $\frac{42}{63}$ & $\frac{9 \frac{6}{8}}{8}$ & $\frac{163}{143}$ & $\frac{24}{198}$ & & & & & 0.667 & 0.970 & 1. 133 & 1. 231 & \\
\hline I & & & & & $\frac{7}{6} \frac{2}{6}$ & $\frac{13 \frac{8}{14}}{14}$ & $\frac{216}{196}$ & $\frac{306}{258}$ & & & & & 0.727 & 0.965 & 1. 108 & 1. 200 \\
\hline
\end{tabular}

${ }^{4} \mathrm{~S}_{2}-{ }^{4} \mathrm{~S}_{2}{ }_{2}(0.00), 2.00$.

${ }^{4} \mathrm{~S}_{2}-{ }^{4} \mathrm{P}_{1}(0.33), \mathbf{1 . 6 7}, 2.33$.

${ }^{4} \mathrm{~S}_{2}-{ }^{4} \mathrm{P}_{2}(0.13,0.40), 1.60,1.8 \gamma, 2.13$.

${ }^{4} \mathrm{~S}_{2}-{ }^{4} \mathrm{P}_{3}(\boldsymbol{\theta} .20,0.60), \mathbf{1 . 0 0}, 1.40,1.80,2.20$.

${ }^{4} \mathrm{~S}_{2}-{ }^{4} \mathrm{D}_{1}^{\prime}{ }_{1}(1.00), 1.00,3.00$.

${ }^{4} \mathrm{~S}_{2}-4 \mathrm{D}_{2}{ }_{2}(0.40,1.20), 0.80,1.60,2.40$.

${ }^{4} \mathrm{~S}_{2}-4 \mathrm{D}_{3}^{\prime}{ }_{3}$ (0.31, 0.94), 0.43, 1.06, 1.68, 2.31.

${ }_{4}^{4} \mathrm{~S}_{2}-{ }^{4} \mathrm{~F}_{2}(0.80,2.40),-0.40,+1.20,2.80$.

${ }_{4}^{4} \mathrm{~S}_{2}-{ }^{4} \mathrm{~F}_{3}(0.49,1.46),-0.43,+0.54,1.51,2.48$.

${ }^{4} \mathrm{P}_{1}{ }^{4} \mathrm{P}_{1}{ }_{1}(0.00), 2.67$.

$\left.{ }^{4} \mathrm{P}_{1}{ }^{-4} \mathrm{P}_{2}{ }^{4} \mathrm{P}^{\prime}{ }_{1}\right\}$ (0.47), 1.27, 2.20 .

${ }^{4} \mathrm{P}_{2}-4 \mathrm{P}^{\prime}{ }_{2}(0.00), 1.73$.

$\left.{ }^{4} \mathrm{P}_{2} \mathrm{P}_{3}{ }^{4} \mathrm{P}^{\prime} \mathrm{P}_{3}{ }_{3}{ }_{2}\right\}(0.07,0.20), 1.40,1.54,1.67,1.80$.

' $\mathrm{P}_{3}{ }^{4} \mathrm{P}_{3}^{\prime}{ }_{3}(0.00), 1.60$.

${ }^{4} \mathrm{P}_{1}{ }^{-4} \mathrm{D}_{1}$ (1.33), 1.33 .

${ }^{4} \mathrm{P}_{1}{ }^{4} \mathrm{D}_{2}(0.73), 0.47,1.93$.

${ }^{4} \mathrm{P}_{2}-4 \mathrm{D}_{1}(0.87), 0.87, \mathbf{2 . 6 0}$.

${ }^{4} \mathrm{P}_{2}{ }^{4} \mathrm{D}_{2}(0.27,0.80), 0.93,1.47,2.00$.

${ }^{4} \mathrm{P}_{2}-{ }^{4} \mathrm{D}_{3}(0.18,0.54), 0.83,1.19,1.55,1.92$.

${ }^{4} \mathrm{P}_{3}-4 \mathrm{D}_{2}(\mathbf{0 . 2 0}, 0.60), 1.00,1.40,1.80,2.20$.

${ }^{4} \mathrm{P}_{3}{ }^{4} \mathrm{D}_{3}(0.11,0.34,0.57), 1.03,1.26,1.48,1.72,1.94$.

${ }^{4} \mathrm{P}_{3}{ }^{-4} \mathrm{D}_{4}(0.09,0.26,0.43), 1.09,1.17,1.34,1.52,1.68,1.86$.

${ }^{4} \mathrm{P}_{1}{ }^{4} \mathrm{~F}_{2}{ }_{2}(1.13), 0.73,1.53$.

${ }^{4} \mathrm{P}_{2}-4 \mathrm{~F}_{2}{ }_{2}(0.67,2.00),-0.27,+\mathbf{1 . 0 7}, 2.40$.

${ }^{4} \mathrm{P}_{2}{ }^{4} \mathrm{~F}^{\prime}{ }_{3}(\mathbf{0 . 3 5}, 1.06),-\mathbf{0 . 0 3},+0.68,1.38,2.09$.

${ }^{4} \mathrm{P}_{3}-4 \mathrm{~F}^{\prime}{ }_{2}(0.60,1.80),-0.20,+1.00,2.20,3.40$.

${ }^{4} \mathrm{P}_{3-4}{ }^{4} \mathrm{~F}_{3}{ }_{3}(0.29,0.86,1.43), 0.17,0.74,1.31,1.89,2.46$.

${ }^{4} \mathrm{P}_{3}{ }^{-4} \mathrm{~F}_{4}^{\prime}(0.18,0.54,0.90), 0.35,0.70,1 . \mathrm{C} 9,1.42,1.78,2.14$.

$4914^{\circ}-28-2$ 
TABLE 2.-Theoretical Zeeman effects (quartet system)-Continued

${ }^{4} \mathrm{P}_{2}-{ }^{4} \mathrm{G}_{3}(\mathbf{0 . 5 8}, 1.74),-1.17,-0.01,+1.15,2.31$.

${ }^{4} \mathrm{P}_{3}{ }^{4} \mathrm{G}_{3}(0.52,1.54,2.57),-0.97,+0.06,1.08,2.11,3.14$.

${ }^{4} \mathrm{P}_{3}-{ }^{4} \mathrm{G}_{4}(0.31,0.93,1.54),-0.55,+0.06,0.68,1.29,1.91,2.52$.

${ }^{4} \mathrm{D}_{1}{ }^{4} \mathrm{D}_{1}{ }_{1}(0.00), 0.00$, unaffected.

$\left.{ }^{4} \mathrm{D}_{1-4}{ }^{4} \mathrm{D}_{2}^{-4} \mathrm{D}_{2}^{\prime}{ }_{1}\right\}(0.60), 0.60,1.80$.

${ }^{4} \mathrm{D}_{2}-4 \mathrm{D}^{\prime}{ }_{2}(0.00), 1.20$.

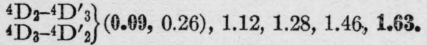

${ }_{4}^{4} \mathrm{D}_{3}-4 \mathrm{D}_{3}^{\prime}(0.00), 1.37$.

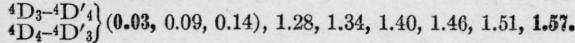

${ }^{4} \mathrm{D}_{4}{ }^{4} \mathrm{D}^{\prime}{ }_{4}(0.00), 1.43$.

${ }^{4} \mathrm{D}_{1}-4 \mathrm{~F}_{2}(0.20), 0.20,0.60$.

${ }^{4} \mathrm{D}_{2}-4 \mathrm{~F}_{2}(0.40,1.20), 0.00,0.80,1.60$.

${ }^{4} \mathrm{D}_{2}-4 \mathrm{~F}_{3}(0.09,0.26), 0.77,0.94,1.12,1.29$.

${ }^{4} \mathrm{D}_{3}{ }^{-4} \mathrm{~F}_{2}(0.49,1.46),-0.09,+\theta .88,1.86,2.83$.

${ }_{4}^{4} \mathrm{D}_{3}-{ }^{4} \mathrm{~F}_{3}(0.17,0.51,0.86), 0.51,0.86,1.20,1.54,1.89$.

$\left.{ }^{4} \mathrm{D}_{3}-4 \mathrm{~F}_{4}(0.07), 0.20,0.33\right), 0.91,1.04,1.17,1.30,1.43,1.57$.

${ }^{4} \mathrm{D}_{4}{ }^{-4} \mathrm{~F}_{3}(\mathbf{0 . 2 0}, 0.60,1.00), 0.43,0.83,1.23,1.63,2.03$, 2.43.

${ }^{4} \mathrm{D}_{4}{ }^{-4} \mathrm{~F}_{4}(0.10,0.29,0.48,0.67), 0.76,0.95,1.14,1.33,1.52,1.72,1.90$.

${ }^{4} \mathrm{D}_{4}{ }^{-4} \mathrm{~F}_{5}(\mathbf{0 . 0 5}, 0.14,0.24,0.33), \mathbf{1 . 0 0}, 1.10,1.19,1.29,1.38,1.48,1.57,1.67$.

${ }_{4}^{4} \mathrm{D}_{2-4}{ }^{4} \mathrm{G}_{3}{ }_{3}(\mathbf{0 . 3 1}, 0.94),-0.37,+0.26,0.89,1.52$.

${ }^{4} \mathrm{D}_{3}{ }^{4} \mathrm{G}^{\prime}{ }_{3}(0.40,1.20,2.00),-0.63,+0.17,0.97,1.77,2.57$.

${ }^{4} \mathrm{D}_{3}{ }^{4} \mathrm{G}^{\prime}{ }_{4}(0.19,0.58,0.97), 0.02,0.40,0.79,1.18,1.57,1.95$.

${ }^{4} \mathrm{D}_{4^{-4}} \mathrm{G}^{\prime}{ }_{3}(0.43,1.29,2.14),-0.71,+0.14,1.00,1.85,2.71,3.57$.

${ }^{4} \mathrm{D}_{4}{ }^{-4} \mathrm{G}^{\prime}{ }_{4}(0.22,0.67,1.11,1.56),-0.13,+0.32,0.76,1.21,1.65,2.10,2.54$.

${ }^{4} \mathrm{D}_{4^{-4}} \mathrm{G}^{\prime}{ }_{5}(\mathbf{0 . 1 3}, 0.38,0.64,0.90), 0.27,0.53,0.79,1.04,1.30,1.56,1.81,2.07$.

${ }^{4} \mathrm{D}_{3}{ }^{4} \mathrm{H}_{4}(0.35,1.06,1.76),-1.69,-0.39,+0.31,1.02,1.73,2.42$.

${ }^{4} \mathrm{D}_{4}{ }^{-4} \mathrm{H}_{4}(0.38,1.14,1.90,2.66),-1.24,-0.48,+0.29,1.05,1.81,2.57,3.33$.

${ }^{4} \mathrm{D}_{4}{ }^{4} \mathrm{H}_{5}(0.23,0.69,1.15,1.61),-0.64,-0.18,+0.28,0.74,1.20,1.66,2.12,2.58$.

${ }^{4} \mathrm{~F}_{2}-4 \mathrm{~F}^{\prime}{ }_{2}(0.00), 0.4 \mathrm{C}$.

$\left.{ }_{4}^{4} \mathrm{~F}_{2} \mathrm{~F}_{3}-4 \mathrm{~F}^{\prime} \mathrm{F}^{\prime}{ }_{3}\right\}$ (0.31, 0.94), 0.09, 0.71, 1.34, 1.97 .

${ }^{4} \mathrm{~F}_{3}-4 \mathrm{~F}_{3}{ }_{3}(0.00), 1.03$.

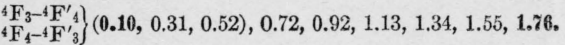

${ }^{4} \mathrm{~F}_{4}{ }^{-4} \mathrm{~F}_{4}^{\prime}(0.00), 1.24$.

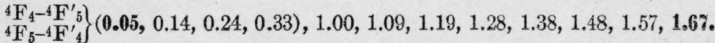

${ }^{4} \mathrm{~F}_{5}{ }^{-4} \mathrm{~F}_{5}^{\prime}(0.00), 1.33$.

${ }^{4} \mathrm{~F}_{2}-{ }^{4} \mathrm{G}_{3}(\mathbf{0 . 0 9}, 0.26), 0.31,0.49,0.66,0.83$.

${ }^{4} \mathrm{~F}_{3}-{ }^{4} \mathrm{G}_{3}(0.23,0.69,1.14),-0.11,+0.34,0.80,1.26,1.72$.

${ }^{4} \mathrm{~F}_{3}{ }^{-4} \mathrm{G}_{4}(0.02,0.07,0.11), 0.87,0.92,0.96,1.01,1.05,1.10$.

${ }^{4} \mathrm{~F}_{4}{ }^{-4} \mathrm{G}_{3}(0.33,1.00,1.67),-0.43,+0.24,0.90,1.57,2.24,2.90$.

${ }^{4} \mathrm{~F}_{4^{-4}} \mathrm{G}_{4}(0.13,0.38,0.64,0.89), 0.35,0.60,0.86,1.11,1.37,1.62,1.87$.

${ }^{4} \mathrm{~F}_{4}{ }^{-4} \mathrm{G}_{5}(0.03,0.10,0.17,0.23) 0.94,1.01,1.07,1.14,1.21,1.27,1.34,1.40$.

${ }^{4} \mathrm{~F}_{5}{ }^{-4} \mathrm{G}_{4}(\mathbf{0 . 1} \mathrm{z}, 0.52,0.87,1.22), 0.11,0.46,0.81,1.16,1.51,1.86,2,20,2.56$.

${ }^{4} \mathrm{~F}_{5}{ }^{-4} \mathrm{G}_{5}(0.08,0.24,0.40,0.57,0.73), 0.61,0.77,0.93,1.09,1.25,1.42,1.58,1,74,1.90$.

${ }^{4} \mathrm{~F}_{3^{-}}{ }^{4} \mathrm{G}_{6}(0.03,0.09,0.15,0.21,0.27), 1.00,1,061.12,1.18,1.24,1.30,1.36,1.42,1.49,1.55$.

${ }_{4}^{4} \mathrm{~F}_{3}-4 \mathrm{H}_{4}^{\prime}(0.18,0.54,0.90),-0.24,+0.12,0.49,0.85,1.21,1.57$.

${ }^{4} \mathrm{~F}_{4}{ }^{-4} \mathrm{H}_{4}^{\prime}(0.29,0.86,1.43,2.00),-0.76,-0.19,+0.38,0.95,1.52,2.10,2.68$.

${ }^{4} \mathrm{~F}_{4}{ }^{-4} \mathrm{H}_{5}{ }_{5}(\mathbf{0 . 1 3}, 0.40,0.67,0.94), \mathbf{0 . 0 3}, 0.30,0.57,0.84,1.10,1.37,1.64,1.91$.

${ }^{4} \mathrm{~F}_{5-4}{ }^{4} \mathrm{H}_{4}{ }_{4}(0.33,1.00,1.67,2.33),-1.00,-0.33,+0.33,1.00,1.67,2.33,3.00,3.67$.

${ }^{4} \mathrm{~F}_{5}{ }^{-4} \mathrm{H}_{5}{ }_{5}(0.18,0.55,0.91,1.27,1.64),-0.30,+0.06,0.42,0.79,1.15,1.51,1.88,2.24,2.61$.

${ }^{4} \mathrm{~F}_{5}{ }^{-4} \mathrm{H}_{6}{ }_{6}(\mathbf{0 . 1 0}, 0.30,0.50,0.70,0.90), 0.23,0.43,0.63,0.83,1.03,1.23,1.43,1.63,1.83,2.03$.

${ }^{4} \mathrm{~F}_{4^{-4}} \mathrm{I}_{5}(\mathbf{0 . 2 6}, 0.77,1.28,1.79),-1.06,-0.55,-0.04,+0.47,0.98,1.49,2.00,2.52$.

${ }_{4}^{4} \mathrm{~F}_{5}{ }^{4} \mathrm{I}_{5}(0.30,0.91,1.52,2.13,2.24),-1.40,-0.79,-0.19,+0.42,1.03,1.64,2.24,2.85,3.46$.

${ }^{4} \mathrm{~F}_{5}{ }^{-4} \mathrm{I}_{6}(0.18,0.55,0.92,1.29,1.66),-0.69,-0.32,+0.04,0.42,0.78,1.15,1.52,1.88,2.25,2.62$. 
TABLE 2.-Theoretical Zeeman effects (quartet system)-Continued

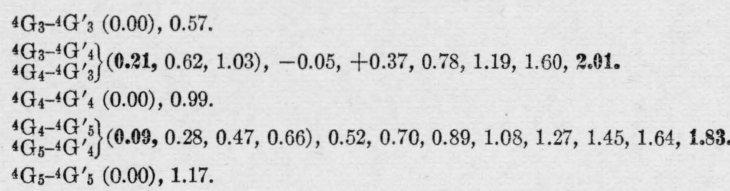

$\left.{ }^{4} \mathrm{G}_{5}^{-4} \mathrm{G}_{6}^{\prime}{ }_{6} \mathrm{G}_{6}^{-4} \mathrm{G}_{5}{ }_{5}\right\}(\mathbf{0 . 0 5}, 0.15,0.25,0.35,0.45), 0.82,0.92,1.02,1.12,1.22,1.32,1.42,1.53,1.63,1$. \%ง

${ }^{4} \mathrm{G}_{6}-{ }^{4} \mathrm{G}_{6}^{\prime}(0.00), 1.27$.

${ }^{4} \mathrm{G}_{3}-{ }^{4} \mathrm{H}_{4}(0.05,0.14,0.24), 0.43,0.52,0.62,0.71,0.81,0.90$.

${ }^{4} \mathrm{G}_{4}{ }^{4} \mathrm{H}_{4}(0.16,0.48,0.79,1.11),-0.13,+0.19,0.51,0.83,1.14,1.46,1.78$.

${ }^{4} \mathrm{G}_{4}{ }^{-4} \mathrm{H}_{5}(\mathbf{0 . 0 1}, 0.02,0.04,0.05), 0.92,0.93,0.95,0.96,0.98,0.99,1.00,1.02$.

${ }^{4} \mathrm{G}_{5}{ }^{-4} \mathrm{H}_{4}(\mathbf{0 . 2 5}, 0.76,1.26,1.77),-0.60,-0.09,+0.41,0.92,1.42,1.93,2.44,2.94$.

${ }^{4} \mathrm{G}_{5}-{ }^{4} \mathrm{H}_{5}(0.10,0.30,0.50,0.71,0.91), 0.26,0.46,0.67,0.87,1.07,1.27,1.47,1.68,1.88$.

${ }^{4} \mathrm{G}_{5}{ }^{4} \mathrm{H}_{6}(\mathbf{0 . 0 2}, 0.06,0.10,0.14,0.17), 0.96,1.00,1.03,1.07,1.11,1.15,1.19,1.23,1.27,1.31$.

${ }^{4} \mathrm{G}_{6}{ }^{-4} \mathrm{H}_{5}(0.15,0.45,0.76,1.06,1.36),-0.09,+0.21,0.51,0.82,1.12,1.42,1.73,2.03,2,33,2.64$.

${ }^{4} \mathrm{G}_{6}{ }^{-4} \mathrm{H}_{6}(0.07,0.21,0.35,0.49,0.63,0.77), 0.50,0.64,0.78,0.92,1.06,1.20,1.34,1.48,1.62,1.76,1.90$.

${ }^{4} \mathrm{G}_{6}{ }^{-4} \mathrm{H}_{7}(0.02,0.06,0.10,0.15,0.19,0.23), 1.00,1.04,1.08,1.13,1.17,1.21,1.25,1.29,1.33,1.38,1.42,1.46$.

${ }^{4} \mathrm{G}_{4}-\mathrm{I}^{\prime}{ }_{5}(\mathbf{0 . 1 3}, 0.39,0.64,0.90),-0.17,+0.08,0.34,0.60,0.86,1.11,1.37,1.62$.

${ }^{4} \mathrm{G}_{5}{ }^{-4} \mathrm{I}_{5}{ }_{5}(0.22,0.67,1.11,1.56,2.00),-0.83,-0.38,+0.06,0.50,0.95,1.39,1.84,2.28,2.73$.

${ }^{4} \mathrm{G}_{5}{ }^{4} \mathrm{I}^{\prime}{ }_{6}(\mathbf{0 . 1 0}, 0.31,0.52,0.73,0.93), 0.04,0.24,0.45,0.66,0.86,1.07,1.27,1.48,1.69,1.89$.

${ }^{4} \mathrm{G}_{6}{ }^{-4} \mathrm{I}_{5}{ }_{5}(0.27,0.82,1.36,1.90,2.45),-1.18,-0.64,-0.09,+0.45,1.00,1.55,2.09,2.64,3.18,3.73$.

${ }^{4} \mathrm{G}_{6}-{ }^{4} \mathrm{I}_{6}{ }_{6}(0.15,0.46,0.77,1.08,1.38,1.69),-0.42,-0.11,0.20,0.50,0.81,1.12,1.43,1.73,2.04,2.35,2.66$.

${ }^{4} \mathrm{G}_{6}-{ }^{4} \mathrm{I}^{\prime}{ }_{7}(\mathbf{0 . 0 8}, 0.25,0.41,0.58,0.74,0.91), 0.20,0.36,0.53,0.70,0.86,1.03,1.19,1.35,1.52,1.69,1.85,2.02$.

${ }^{4} \mathrm{H}_{4}{ }^{-4} \mathrm{H}^{\prime}{ }_{4}(0.00), 0.67$.

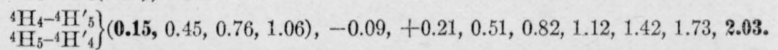

${ }^{4} \mathrm{H}_{5}-{ }^{4} \mathrm{H}_{5}^{\prime}(0.00), 0.97$.

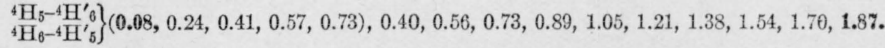

${ }^{4} \mathrm{H}_{6}-{ }^{4} \mathrm{H}_{6}^{\prime}(0.00), 1.13$.

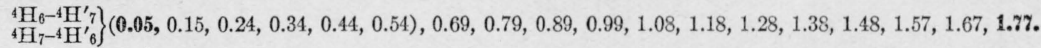

${ }^{4} \mathrm{H}_{7}{ }^{-4} \mathrm{H}^{\prime}{ }_{7}(0.00), 1.23$.

${ }^{4} \mathrm{H}_{4}{ }^{4} \mathrm{I}_{5}(\mathbf{0 . 0 3}, 0.09,0.15,0.21), 0.52,0.58,0.64,0.70,0.76,0.82,0.88,0.94$.

${ }^{4} \mathrm{H}_{5}-\mathrm{I}_{5}(0.12,0.36,0.61,0.85,1.09),-0.12,+0.12,0.36,0.61,0.85,1.09,1.33,1.58,1.82$.

${ }^{4} \mathrm{H}_{5}-{ }^{4} \mathrm{I}_{6}(\mathbf{0 . 0 0}, 0.01,0.01,0.02,0.02), 0.94,0.95,0.95,0.96,0.96,0.97,0.97,0.98,0.98,0.99$.

${ }^{4} \mathrm{H}_{0}{ }^{-4} \mathrm{I}_{5}(0.20,0.61,1.01,1.42,1.83),-0.69,-0.29,+0.12,0.53,0.93,1.33,1.74,2.15,2.55,2.96$.

${ }^{4} \mathrm{H}_{6}{ }^{-4} \mathrm{I}_{6}(0.08,0.25,0.42,0.59,0.75,0.92), 0.21,0.38,0.55,0.71,0.88,1.05,1.22,1.38,1.55,1.72,1.89$.

${ }^{4} \mathrm{H}_{6}{ }^{-4} \mathrm{I}_{7}(\mathbf{0 . 0 1}, 0.04,0.06,0.09,0.11,0.14), 0.97,0.99,1.02,1.04,1.07,1.10,1.12,1.15,1.17,1.20,1.22,1.25$.

${ }^{4} \mathrm{H}_{7}{ }^{-4} \mathrm{I}_{6}(0.13,0.40,0.66,0.93,1.19,1.46),-0.23,+0.03,0.30,0.57,0.83,1.10,1.36,1.63,1.89,2.16,2.43,2.69$.

${ }^{4} \mathrm{H}_{7}{ }^{-4} \mathrm{I}_{7}(0.06,0.18,0.31,0.43,0.55,0.68,0.80), 0.44,0.56,0.68,0.80,0.92,1.05,1.17,1.29,1.41,1.54,1.66,1.78,1.91$.

${ }^{4} \mathrm{H}_{7}{ }^{-4} \mathrm{I}_{8}(0.02,0.05,0.08,0.11,0.14,0.17,0.20), 1.09,1.03,1.06,1.09,1.12,1.15,1.18,1.22,1.25,1.28,1.31,1.34$, $1.37,1.40$.

${ }^{4} \mathrm{I}_{5}-4 \mathrm{I}_{5}{ }_{5}(0.00), 0.73$.

$\left.{ }^{4} \mathrm{I}_{5} \mathrm{I}_{6}^{-4} \mathrm{I}_{6}^{4} \mathrm{I}_{6}^{\prime}{ }_{5}^{\prime}\right\}(0.01,0.36,0.59,0.83,1.07),-0.10,+0.13,0.37,0.61,0.84,1.08,1.32,1.56,1.79,2.03$.

${ }^{4} I_{6}-4 I_{6}(0.00), 0.97$.

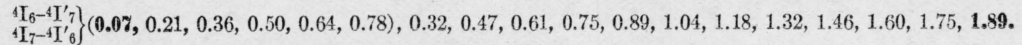

4I $\mathrm{I}_{7}{ }^{4} \mathrm{I}_{7}{ }_{7}(0.00), 1.11$.

$\left.{ }^{4} \mathrm{I}_{7}{ }^{-4} \mathrm{I}^{\prime} 8\right)(0.05,0.14,0.23,0.32,0.42,0.51,0.60), 0.60,0.69,0.78,0.88,0.97,1.06,1.15,1.25,1.34,1.43,1.52,1.62$,

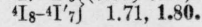

${ }^{4} I_{8}-4 I^{\prime}{ }_{8}(0.00), 1.20$. 
TABLE 3.-Theoretical Zeeman effects (sextet system)

[Landé $g$ values]

\begin{tabular}{|c|c|c|c|c|c|c|c|c|c|c|c|c|c|c|c|c|c|c|}
\hline$l^{j}$ & 1 & 2 & 3 & 4 & 5 & 6 & 7 & 8 & 9 & 1 & , 2 & 3 & 4 & 5 & 6 & 7 & 8 & 9 \\
\hline $\mathrm{s}$ & & & $\frac{7}{35} \frac{0}{5}$ & & & & & & & & & 2. 000 & & & & & & \\
\hline $\mathbf{P}$ & & $\frac{38}{15}$ & $\frac{8}{3} \frac{8}{5}$ & $\frac{108}{63}$ & & & & & & & 2. 400 & 1. 886 & 1. 714 & & & & & \\
\hline $\mathrm{D}$ & $10 \frac{0}{3}$ & $\frac{28}{15}$ & $\frac{58}{35}$ & $1 \frac{10}{6}$ & $\frac{184}{98}$ & & & & & 3. 333 & 1. 867 & 1. 657 & 1. 587 & 1. 556 & & & & \\
\hline $\mathrm{F}$ & $-\frac{3}{3}$ & $\frac{16}{1} \frac{6}{5}$ & $\frac{46}{35}$ & $\frac{89}{83}$ & $\frac{143}{99}$ & $\frac{308}{143}$ & & & & -0.667 & 1. 067 & 1. 314 & 1. 397 & 1.434 & 1.455 & & & \\
\hline G & & is & $\frac{30}{35}$ & $\frac{\pi^{2}}{3}$ & $2 \frac{2}{3} \frac{8}{\partial}$ & $\frac{19}{14} \frac{2}{3}$ & $\frac{978}{13}$ & & & & 0.000 & 0.857 & 1. 143 & 1. 273 & 1. 343 & 1.385 & & \\
\hline H & & & $\frac{1}{3} \frac{9}{5}$ & $\frac{52}{83}$ & $1 \frac{06}{9} \frac{8}{3}$ & $\frac{172}{143}$ & $\frac{250}{195}$ & $\frac{34}{25} \frac{0}{5}$ & & & & 0.286 & 0.825 & 1. 071 & 1. 203 & 1. 282 & 1. 333 & \\
\hline I & & & & $\frac{28}{63}$ & $\frac{8}{9} \frac{3}{3}$ & $\frac{x 48}{143}$ & $\frac{32}{13} \frac{6}{5}$ & $\frac{31}{215}$ & $\frac{4}{3} \frac{1}{2} \frac{3}{3}$ & & & & 0.444 & 0.828 & 1.035 & 1. 159 & 1. 239 & 1. 294 \\
\hline
\end{tabular}

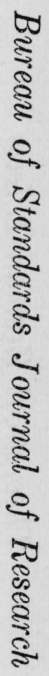


TABLE 3.-Theoretical Zeeman effects (sextet system)-Continued

${ }^{6} \mathrm{~S}_{3}-6 \mathrm{~S}_{3}{ }_{3}(0.00), 2.00$.

${ }^{6} \mathrm{~S}_{3}-6 \mathrm{P}_{2}(\mathbf{0 . 2 0}, 0.60), 1.40,1.80,2.20,2.60$.

${ }^{6} \mathrm{~S}_{3}-6 \mathrm{P}_{3}(0.06,0.17,0.29), 1.71,1.83,1.94,2.06,2.17$.

${ }^{6} \mathrm{~S}_{3}{ }^{-6} \mathrm{P}_{4}(\mathbf{0 . 1 4}, 0.43,0.71), 1.00,1.29,1.57,1.86,2.14,2.43$.

${ }^{6} \mathrm{~S}_{3}-6 \mathrm{D}_{2}^{\prime}{ }_{2}(\mathbf{0 . 0 7}, 0.20), 1.80,1.93,2.07,2.20$.

${ }^{6} \mathrm{~S}_{3}-6 \mathrm{D}_{3}{ }_{3}(0.17,0.51,0.86), 1.14,1.49,1.83,2.17,2.51$.

${ }^{6} \mathrm{~S}_{3}-6 \mathrm{D}_{4}^{\prime}(0.21,0.62,1.03), 0.56,0.97,1.38,1.79,2.21,2.62$.

${ }^{6} \mathrm{~S}_{3}-6 \mathrm{~F}_{2}(\mathbf{0 . 4 7}, 1.40), \mathbf{0 . 6 0}, 1.53,2.46,3.40$.

${ }^{6} \mathrm{~S}_{3}{ }^{-6} \mathrm{~F}_{3}(0.34,1.03, \mathbf{1 . 7 1}), 0.29,0.97, \mathbf{1 . 6 6}, 2.34,3.03$.

${ }^{6} \mathrm{~S}_{3}{ }^{6} \mathrm{~F}_{4}(0.30,0.90,1.50),-0.11,+0.50,1.10,1.70,2.30,2.90$.

${ }^{6} \mathrm{P}_{2}-6 \mathrm{P}_{2}^{\prime}(0.00), 2.40$.

$\left.{ }^{6} \mathrm{P}_{2}-6 \mathrm{P}_{3}^{\prime} \mathrm{P}_{3}^{\prime}{ }_{3}\right\}$ (0.26, 0.77), 1.11, 1.63, 2.14, 2.66.

${ }^{6} \mathrm{P}_{3}-6 \mathrm{P}^{\prime}{ }_{3}(0.00), 1.89$.

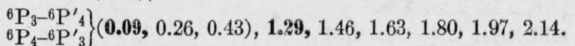

${ }^{6} \mathrm{P}_{1}-6 \mathrm{P}_{4}^{\prime}(0.00), 1.71$.

${ }^{6} \mathrm{P}_{2}-{ }^{6} \mathrm{D}_{1}(0.47), 1.93,2.87$.

${ }^{6} \mathrm{P}_{2}{ }^{6} \mathrm{D}_{2}(0.27,0.80), 1.60,2.13,2.67$.

${ }^{6} \mathrm{P}_{2}-{ }^{6} \mathrm{D}_{3}(0.37,1.12), 0.54,1.29,2.03,2.77$.

${ }^{6} \mathrm{P}_{3}-{ }^{6} \mathrm{D}_{2}(\mathbf{0 . 0 1}, 0.03), 1.86,1.88,1.90,1.92$.

${ }^{6} \mathrm{P}_{3}-{ }^{6} \mathrm{D}_{3}(0.11,0.34, \mathbf{0 . 5} 7), 1.31,1.54,1.77,2.00,2.23$.

${ }^{6} \mathrm{P}_{3}-6 \mathrm{D}_{4}(\mathbf{0 . 1 5}, 0.45,0.75), 0.84,1.14,1.44,1.74,2.03,2.33$.

${ }^{6} \mathrm{P}_{4}-6 \mathrm{D}_{3}(0.03,0.09,0.14), 1.57,1.63,1.68,1.74,1.80,1.86$.

${ }^{6} \mathrm{P}_{4}{ }^{6} \mathrm{D}_{4}(0.06,0.19,0.32,0.44), 1.27,1.40,1.52,1.65,1.78,1.91,2.03$.

${ }^{6} \mathrm{P}_{4}{ }^{-6} \mathrm{D}_{5}(0.08,0.24,0.40,0.56), 1.00,1.16,1.32,1.48,1.63,1.79,1.95,2.11$.

${ }^{6} \mathrm{P}_{2}-{ }^{6} \mathrm{~F}^{\prime}{ }_{1}(1.53), 0.87,3.94$.

${ }^{6} \mathrm{P}_{2}-6 \mathrm{~F}^{\prime}{ }_{2}(0.67,2.00), 0.4,0,1.73,3.07$.

${ }^{6} \mathrm{P}_{2}-{ }^{6} \mathrm{~F}^{\prime}{ }_{3}(0.54,1.63),-0.31,+0.77,1.86,2.94$.

${ }^{6} \mathrm{P}_{3}-6 \mathrm{~F}^{\prime}{ }_{2}(0.41,1.23), 0.66,1.47,2.29,3.11$.

${ }^{6} \mathrm{P}_{3}-6 \mathrm{~F}_{3}{ }_{3}(0.29,0.86,1.43), 0.46,1.03,1.60,2.17,2.74$.

${ }^{6} \mathrm{P}_{3}-6 \mathrm{~F}^{\prime}{ }_{4}(\mathbf{0 . 2 4}, 0.73,1.22), 0.17,0.66,1.15,1.64,2.13,2.62$.

${ }^{6} \mathrm{P}_{4}-6 \mathrm{~F}^{\prime}{ }_{3}(0.20,0.60,1.00), 0.71,1.11,1.51,1.91,2.32$, 2.72.

${ }^{6} \mathrm{P}_{4}{ }^{-6} \mathrm{~F}^{\prime}{ }_{4}(0.16,0.48,0.79,1.11), 0.60,0.92,1.24,1.55,1.87,2.19,2.51$.

${ }^{6} \mathrm{P}_{4}{ }^{-6} \mathrm{~F}_{5}^{\prime}(0.14,0.42,0.70,0.98), 0.45,0.73,1.01,1.29,1.58,1.85,2.13,2.42$.

${ }^{6} \mathrm{P}_{2}-{ }^{6} \mathrm{G}_{2}(1.20,3.60),-1.20,+1.20,3.60$.

${ }^{6} \mathrm{P}_{2}-6 \mathrm{G}_{3}(\mathbf{0 . 7 7}, 2.31),-1.46,+0.09,1.63,3.16$.

${ }^{6} \mathrm{P}_{3}{ }^{-6} \mathrm{G}_{2}(0.94,2.82),-0.94,+0.94,2.82,4.71$.

${ }^{6} \mathrm{P}_{3}-{ }^{6} \mathrm{G}_{3}(0.51,1.53,2.58),-0.68,+0.35,1.37,2.40,3.42$.

${ }^{6} \mathrm{P}_{3}{ }^{-6} \mathrm{G}_{4}(\mathbf{0 . 3 7}, 1.11,1.86),-0.72,+0.03,0.77,1.51,2.25,3.01$.

${ }^{6} \mathrm{P}_{4}{ }^{-6} \mathrm{G}_{3}(0.43,1.29,2.14),-0.43,+0.43,1.28,2.14,3.00,3.86$.

${ }^{6} \mathrm{P}_{4}-6 \mathrm{G}_{4}(0.29,0.86,1.43,2.00),-0.29,+0.29,0.86,1.43,2.00,2.57,3.14$.

${ }^{6} \mathrm{P}_{4}{ }^{-6} \mathrm{G}_{5}(0.22,0.66,1.10,1.55),-0.27,+0.17,0.61,1.05,1.49,1.93,2.37,2.82$.

${ }^{6} \mathrm{D}_{1}{ }^{-6} \mathrm{D}_{1}^{\prime}(0.00), 3.33$.

$\left.{ }^{6} \mathrm{D}_{1}{ }^{6} \mathrm{D}_{2}^{-6} \mathrm{D}_{2}^{\prime} \mathrm{D}_{1}^{\prime}\right\}(0.73), 1.14,2.60$.

${ }^{6} \mathrm{D}_{2}{ }^{6} \mathrm{D}_{2}^{\prime}(0.00), 1.87$.

$\left.{ }^{6} \mathrm{D}_{2}{ }^{6} \mathrm{D}_{3}{ }^{-6} \mathrm{D}_{3}^{\prime}{ }_{2}^{\prime}\right\}(0.10,0.31), \mathbf{1 . 3 4}, 1.55,1.76,1.97$.

${ }^{6} \mathrm{D}_{3}-{ }^{6} \mathrm{D}_{3}^{\prime}(0.00), 1.66$.

$\left.{ }^{6} \mathrm{D}_{3} \mathrm{D}_{4}^{-6} \mathrm{D}_{4}^{\prime} \mathrm{D}_{3}^{\prime}\right\}(0.03,0.10,0.17), 1.41,1.48,1.55,1.62,1.69,1.76$.

${ }^{6} \mathrm{D}_{4^{-6}} \mathrm{D}_{4}^{\prime}(0.00), 1.59$.

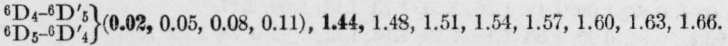

${ }^{6} \mathrm{D}_{5}{ }^{-6} \mathrm{D}_{5}^{\prime}(0.00), 1.55$.

${ }^{6} \mathrm{D}_{1-6}{ }^{-6} \mathrm{~F}_{1}(2.00), 1.33$.

${ }^{6} \mathrm{D}_{1}{ }^{-6} \mathrm{~F}_{2}(1.13), 0.07,2.20$.

${ }^{6} \mathrm{D}_{2}{ }^{6} \mathrm{~F}_{1}(1.27), 0.60,3.13$.

${ }^{6} \mathrm{D}_{2}-{ }^{6} \mathrm{~F}_{2}(0.40,1.20), 0.67,1.47,2.27$.

${ }^{6} \mathrm{D}_{2}{ }^{-6} \mathrm{~F}_{3}(0.28,0.83), 0.49,1.04,1.59,2.14$.

${ }^{6} \mathrm{D}_{3}{ }^{-6} \mathrm{~F}_{2}(0.29,0.88), 0.77,1.36,1.95,2.51$.

${ }^{6} \mathrm{D}_{3}{ }^{-6} \mathrm{~F}_{3}(0.17,0.51,0.86), 0.80,1.14,1.49,1.83,2.17$.

${ }^{6} \mathrm{D}_{3}{ }^{-6} \mathrm{~F}_{4}(0.13,0.39,0.65), 0.75,1.01,1.27,1.53,1.79,2.05$. 
TABLE 3.-Theoretical Zeeman effects (sextet system)-Continued

${ }^{6} \mathrm{D}_{4^{-6}} \mathrm{~F}_{3}(0.14,0.41,0.68), 0.90,1.18,1.45,1.73,2.00$, 2.2\%

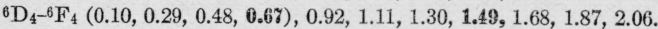

${ }^{6} \mathrm{D}_{4}{ }^{-6} \mathrm{~F}_{5}(0.08,0.23,0.38,0.53), 0.90,1.05,1.20,1.36,1.51,1.66,1.82,1.97$.

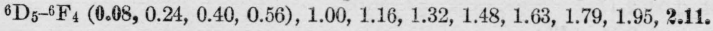

${ }^{6} \mathrm{D}_{5}{ }^{-6} \mathrm{~F}_{5}(0.06,0.18,0.30,0.42,0.55), 1.01,1.13,1.25,1.37,1.49,1.62,1.74,1.86,1.98$.

${ }^{6} \mathrm{D}_{5}{ }^{-6} \mathrm{~F}_{6}(0.05,0.15,0.25,0.35,0.45), 1.60,1.10,1.20,1.30,1.40,1.51,1.61,1.71,1.81,1.91$.

${ }^{8} \mathrm{D}_{1}-{ }^{6} \mathrm{G}_{2}^{\prime}(1.67), 1.67$.

${ }^{6} \mathrm{D}_{2}{ }^{-6} \mathrm{G}_{2}^{\prime}{ }_{2}(0.93,2.80),-0.93,+0.93,2.80$.

${ }^{6} \mathrm{D}_{2}{ }^{-6} \mathrm{G}_{3}^{\prime}(0.50,1.51),-0.66$, $+0.35,1.36,2.37$.

${ }^{6} \mathrm{D}_{3}{ }^{-6} \mathrm{G}_{2}^{\prime}{ }_{2}(0.83,2.48), 0.83,2.48,4.14$.

${ }^{6} \mathrm{D}_{3}{ }^{-6} \mathrm{G}^{\prime}{ }_{3}(0.40,1.20,2.00),-0.34,+0.46,1.26,2.06,2.88$.

${ }^{6} \mathrm{D}_{3}{ }^{-6} \mathrm{G}_{4}^{\prime}(0.26,0.77,1.28),-0.14,+0.37,0.88,1.40,1.91,2.43$.

${ }^{6} \mathrm{D}_{4}{ }^{-6} \mathrm{G}^{\prime}{ }_{3}(0.36,1.10,1.83),-0.24,+0.49,1.22,1.95,2.68,3.41$.

${ }^{6} \mathrm{D}_{4}{ }^{6} \mathrm{GG}^{\prime}{ }_{4}(0.22,0.67,1.11,1.56), 0.03,0.48,0.92,1.36,1,81,2.25,2.70$.

${ }^{6} \mathrm{D}_{4}{ }^{-6} \mathrm{G}_{5}^{\prime}(0.16,0.47,0.79,1.10), 0.17,0.49,0.80,1.12,1.43,1.74,2.06,2.37$.

${ }^{8} \mathrm{D}_{5}{ }^{-6} \mathrm{G}_{4}{ }_{4}(0.21,0.62,1.03,1.44), 0.11,0.52,0.94,1.35,1.76,2.18,2.59,3.00$.

${ }^{6} \mathrm{D}_{5}{ }^{-6} \mathrm{G}_{5}^{\prime}(0.14,0.42,0.71,0.99,1.27), 0.28,0.57,0.85,1.13,1.41,1.70,1.98,2.26,2.54$.

${ }^{6} \mathrm{D}_{6}{ }^{-6} \mathrm{G}_{6}^{\prime}(0.11,0.32,0.53,0.74,0.96), 0.38,0.60,0.81,1.02,1.24,1.45,1.66,1.88,2.09,2.30$.

${ }^{8} \mathrm{D}_{2}{ }^{-6} \mathrm{H}_{3}(0.79,2,37),-2.08,-0.50,+1.08,2.66$.

${ }^{6} \mathrm{D}_{3}{ }^{6} \mathrm{H}_{3}(0.69,2.06,3.42),-1.77,-0.40,+0.97,2.34,3.71$.

${ }^{6} \mathrm{D}_{3}{ }^{-6} \mathrm{H}_{4}(0.42,1.25,2.08),-1.25,-0.42,+0.41,1.24,2.07,2.90$.

${ }^{6} \mathrm{D}_{4}{ }^{6} \mathrm{H}_{3}(0.65,1.95,3.25),-1.67,-0.36,+0.94,2.24,3.54,4.84$.

${ }^{6} \mathrm{D}_{4}{ }^{-6} \mathrm{H}_{4}(0.38,1.14,1.90,2.67),-1.08,-0.32,+0.44,1.21,1,97,2.73,3.49$.

${ }^{6} \mathrm{D}_{4}{ }^{6} \mathrm{H}_{5}(\mathbf{0 . 2 6}, 0.78,1.29,1.81),-0.74,-0.22,+0.30,0.81,1.33,1.85,2.36,2.88$.

${ }^{6} \mathrm{D}_{5}{ }^{-6} \mathrm{H}_{4}(0.37,1.10,1.83,2.56),-1.00,-0.27,+0.46,1.19,1.92,2.65,3.38,4.11$.

${ }^{6} \mathrm{D}_{5}{ }^{-6} \mathrm{H}_{5}(0.24,0.73,1.21,1.70,2.18),-0.63,-0.14,+0.34,0.83,1.31,1.80,2.28,2.77,3.25$.

${ }^{6} \mathrm{D}_{5}{ }^{-6} \mathrm{H}_{6}(0.18,0.53,0.88,1.23,1.58),-0.38,-0.03,+0.32,0.67,1.03,1.38,1.73,2.08,2.44,2.79$.

${ }^{6} \mathrm{~F}_{1}{ }^{6} \mathrm{~F}^{\prime}{ }_{1}(0.00), 0.67$.

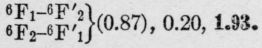

${ }^{6} \mathrm{~F}_{2}{ }^{-6} \mathrm{~F}^{\prime}{ }_{2}(0.00), 1.07$.

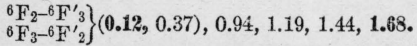

${ }^{6} \mathrm{~F}_{3}-6 \mathrm{~F}^{\prime}{ }_{3}(0.00), 1.31$.

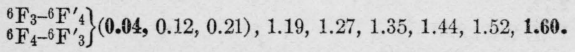

${ }^{6} \mathrm{~F}_{4}{ }^{-6} \mathrm{~F}^{\prime}{ }_{4}(0.00), 1.40$.

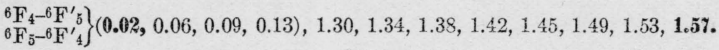

${ }^{6} \mathrm{~F}_{5}{ }^{-6} \mathrm{~F}^{\prime}{ }_{5}(0.00), 1.43$.

$\left.{ }^{6} \mathrm{~F}_{5^{-6} \mathrm{~F}_{6}-6 \mathrm{~F}^{\prime} \mathrm{F}^{\prime}{ }_{5}}\right\}(0.01,0.03,0.05,0.07,0.09), 1.36,1.38,1.40,1.42,1.44,1.46,1.48,1.50,1.52,1.54$.

${ }^{6} \mathrm{~F}_{6}-{ }^{6} \mathrm{~F}^{\prime}{ }_{6}(0.00), 1.45$.

${ }^{6} \mathrm{~F}_{1}-{ }^{6} \mathrm{G}_{2}(0.33), 0.33$.

${ }^{6} \mathrm{~F}_{2}{ }^{-6} \mathrm{G}_{2}(0.53,1.60),-0.53,+0.53,1.60$.

${ }^{6} \mathrm{~F}_{2}{ }^{-6} \mathrm{G}_{3}(0.10,0.32), 0.54,0.75,0.96,1.17$.

${ }^{6} \mathrm{~F}_{3}-{ }^{6} \mathrm{G}_{2}(\boldsymbol{\theta} .23,0.66), 0.63,1.08,1.54, \mathbf{2 . 0 0}$.

${ }^{6} \mathrm{~F}_{3}{ }^{-} \mathrm{G}_{3}(0.23,0.68,1.12), 0.17,0.63,1.08,1,54,2.00$.

${ }^{6} \mathrm{~F}_{3}-{ }^{6} \mathrm{G}_{4}(0.09,0.26,0.43), 0.71,0.88,1.06,1.23,1.40,1.57$.

${ }^{6} \mathrm{~F}_{4}{ }^{-6} \mathrm{G}_{3}(\mathbf{0 . 2 7}, 0.81,1.35), 0.05,0.59,1.13,1.67,2.21,2.75$.

${ }^{6} \mathrm{~F}_{4}{ }^{-6} \mathrm{G}_{4}(0.13,0.38,0.63,0.89), 0.51,0.76,1.01,1.27,1.52,1.78,2.03$.

${ }^{6} \mathrm{~F}_{4}{ }^{-6} \mathrm{G}_{5}(\mathbf{0 . 0 6}, 0.19,0.31,0.43), \mathbf{0 . 8 4}, 0.96,1.08,1.21,1.33,1.46,1.58,1.71$.

${ }^{6} \mathrm{~F}_{5}{ }^{-6} \mathrm{G}_{4}(0.15,0.44,0.73,1.02), 0.41,0.71,1.00,1.29,1.58,1.87,2.16$, 2.45.

${ }^{6} \mathrm{~F}_{5}{ }^{-6} \mathrm{G}_{5}(0.08,0.24,0.40,0.56,0.73), 0.71,0.87,1.03,1.19,1.35,1.52,1.68,1.84,2.00$.

${ }^{6} \mathrm{~F}_{5}{ }^{-6} \mathrm{G}_{6}(0.05,0.14,0.23,0.32,0.41), 0.93,1.02,1.11,1.20,1.30,1.39,1.48,1.57,1.66,1.75$.

${ }^{6} \mathrm{~F}_{6}{ }^{-6} \mathrm{G}_{5}(0.09,0.27,0.45,0.63,0.82), 0.64,0.82,1.00,1.18,1.36,1.55,1.73,1.91,2.09$, 2.27.

${ }^{6} \mathrm{~F}_{6}{ }^{-6} \mathrm{G}_{6}(0.06,0.17,0.28,0.39,0.50,0.62), 0.84,0.95,1.06,1.18,1.29,1.40,1.51,1.62,1.73,1.85,1.96$.

${ }^{6} \mathrm{~F}_{6}{ }^{-6} \mathrm{G}_{7}(0.04,0.10,0.17,0.24,0.31,0.38), 1.00,1.07,1.14,1.21,1.28,1.35,1.42,1.49,1.56,1.63,1.70,1.77$.

${ }^{6} \mathrm{~F}_{2}-{ }^{6} \mathrm{H}^{\prime}{ }_{3}(0.39,1.17),-0.89,-0.10,+0.68,1.46$.

${ }^{6} \mathrm{~F}_{3}-{ }^{6} \mathrm{H}^{\prime}{ }_{3}(0.51,1.54,2.57),-1.26,-0.23,+0.80,1.83,2.86$.

${ }^{6} \mathrm{~F}_{3}-6 \mathrm{H}^{\prime}{ }_{4}(0.24,0.73,1.22),-0.40,+0.09,0.58,1.07,1.56,2.05$.

${ }^{6} \mathrm{~F}_{4}-6 \mathrm{H}^{\prime}{ }_{3}(\mathbf{0 . 5 6}, 1.67,2.78),-1.38,-0.27,+0.84,1.95,3.07,4.17$.

${ }^{6} \mathrm{~F}_{4}-6 \mathrm{H}^{\prime}{ }_{4}(0.29,0.86,1.43,2.00),-0.60,-0.03,+0.54,1.11,1.68,2.25,2.83$.

${ }^{6} \mathrm{~F}_{4}{ }^{-6} \mathrm{H}^{\prime}{ }_{5}(0.16,0.49,0.82,1.14),-0.07,+0.26,0.58,0.91,1.23,1.56,1.88,2.21$.

${ }^{6} \mathrm{~F}_{5}-{ }^{6} \mathrm{H}_{4}^{\prime}(0.30,0.91,1.52,2.13),-0.70,-0.09,+0.52,1.13,1.74,2.34,2.95,3.56$. 
TABLE 3.-Theoretical Zeeman effects (sextet system) - Continued

${ }^{6} \mathrm{~F}_{5}-6 \mathrm{H}^{\prime}{ }_{5}(0.18,0.55,0.91,1.27,1.64),-0.20,+0.16,0.52,0.89,1.25,1.62,1.98,2.34,2.71$.

${ }^{6} \mathrm{~F}_{5}{ }^{-6} \mathrm{H}^{\prime}{ }_{6}(0.12,0.35,0.58,0.81,1.04), 0.16,0.39,0.62,0.86,1.09,1.32,1.55,1.78,2.01,2.24$.

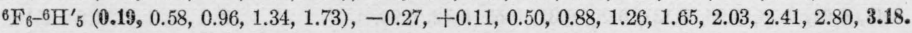

${ }^{6} \mathrm{~F}_{6}{ }^{-}{ }^{6} \mathrm{H}^{\prime}{ }_{6}(0.13,0.38,0.63,0.88,1.13,1.38), 0.07,0.32,0.57,0.82,1.08,1.33,1.58,1.83,2.08,2.33,2.59$.

${ }^{6} \mathrm{~F}_{6}{ }^{-6} \mathrm{H}_{7}^{\prime}(0.09,0.27,0.43,0.60,0.78,0.95) 0.33,0.51,0.68,0.85,1.02,1.20,1.37,1.54,1.71,1.88,2.06,2.23$.

${ }^{6} \mathrm{G}_{2}-\mathrm{e}^{\mathrm{e}}{ }_{2}{ }_{2}(0.00), 0.00$.

$\left.{ }^{6} \mathrm{G}_{2}-{ }^{6} \mathrm{G}_{3}^{\prime}{ }_{3}{ }^{6} \mathrm{G}_{2}^{\prime}\right\}(0.43,1.29),-0.43,+0.43,1.29,2.14$.

${ }^{6} \mathrm{G}_{3}-6 \mathrm{G}^{\prime}{ }_{3}(0.00), 0.86$.

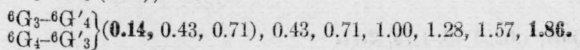

${ }^{6} \mathrm{G}_{4}{ }^{-6} \mathrm{G}^{\prime}{ }_{4}(0.00), 1.14$.

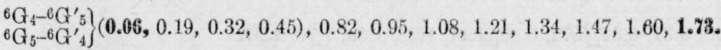

${ }^{8} \mathrm{G}_{5}-{ }^{8} \mathrm{G}_{5}^{\prime}(0.00), 1.27$.

$\left.\begin{array}{l}{ }^{6} \mathrm{G}_{5}-6 \mathrm{G}^{\prime}{ }^{6} \\ { }^{6} \mathrm{G}_{6}-6 \mathrm{G}_{5}{ }_{5}\end{array}\right\}(\mathbf{0 . 0 3}, 0.10,0.17,0.24,0.31), 1.03,1.10,1.17,1.24,1.31,1.38,1.45,1.52,1.59, \mathbf{1 . 6 6}$.

${ }^{6} \mathrm{G}_{6}{ }^{6} \mathrm{G}^{\prime}{ }_{6}(0.00), 1.34$.

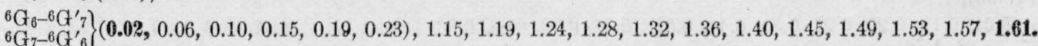

${ }^{6} \mathrm{G}_{7}-{ }^{6} \mathrm{G}^{\prime}{ }_{7}(0.00), 1.38$.

${ }^{6} \mathrm{G}_{2}-{ }^{6} \mathrm{H}_{3}(0.14,0.43),-0.14,+0.14,0.43,0.71$.

${ }^{6} \mathrm{G}_{3}-6 \mathrm{H}_{3}(0.29,0.86,1.43),-0.57,0.00,0.57,1.14,1.71$.

${ }^{6} \mathrm{G}_{3}-6 \mathrm{H}_{4}(\mathbf{0 . 0 2}, 0.05,0.08), \mathbf{0 . 7 5}, 0.78,0.81,0.84,0.87,0.90$.

${ }^{6} \mathrm{G}_{4}{ }^{-6} \mathrm{H}_{3}(0.43,1.29,2.14),-1.00,-0.14,+0.71,1.57,2.43,3.29$.

${ }^{6} \mathrm{G}_{4}{ }^{6} \mathrm{H}_{4}(0.16,0.48,0.79,1.11), 0.03,0.35,0.67,0,98,1.30,1.62,1.93$.

${ }^{6} \mathrm{G}_{4}-{ }^{-6} \mathrm{H}_{5}(\mathbf{0 . 0 4}, 0.11,0.18,0.25), \mathbf{0 . 8 2}, 0.89,0.96,1.03,1.11,1.18,1.25,1.32$.

${ }^{6} \mathrm{G}_{5}-6 \mathrm{G}_{4}(0.22,0.67,1.12,1.56),-0.29,+0.15,0.60,1.05,1.50,1.94,2.38,2.83$.

${ }^{6} \mathrm{G}_{5}{ }^{-6} \mathrm{H}_{5}(0.10,0.30,0.50,0.71, \mathbf{0 . 9 1}), 0.36,0.57,0.77,0.97,1.17,1.37,1.58,1.78,1.98$.

${ }^{6} \mathrm{G}_{5}{ }^{-6} \mathrm{H}_{6}(0.03,0.10,0.17,0.24,0.31), 0.89,0.96,1.03,1.10,1.17,1.24,1.31,1.38,1.45,1.52$.

${ }^{6} \mathrm{G}_{6}{ }^{-6} \mathrm{H}_{5}(\mathbf{0 . 1 4}, 0.41,0.68,0.95,1.22), 0.12,0.39,0.66,0.94,1.21,1.48,1.75,2.02,2.29$, 2.5\%.

${ }^{\circ} \mathrm{G}_{6}{ }^{-6} \mathrm{H}_{6}(0.07,0.21,0.35,0.49,0.63,0.77), 0.57,0.71,0.85,0.99,1.13,1.27,1.41,1.55,1.69,1.83,1.97$.

${ }^{6} \mathrm{H}_{0}{ }^{-6} \mathrm{H}_{7}(\mathbf{0 . 0 3}, 0.09,0.15,0.21,0.27,0.33), 0.95,1.01,1.07,1.13,1.19,1.25,1.31,1.37,1.43,1.49,1.55,1.61$.

${ }^{6} \mathrm{G}_{7}-{ }^{6} \mathrm{H}_{6}(\mathbf{0 . 0 9}, 0.27,0.45,0.64,0.82,1.00), 0.38,0.57,0.75,0.93,1.11,1.29,1.47,1.66,1.84,2.02,2.20$, 2.39.

${ }^{6} \mathrm{G}_{7}-{ }^{6} \mathrm{H}_{7}(0.05,0.15,0.26,0.36,0.56,0.67), 0.72,0.82,0.92,1.02,1.13,1.23,1.33,1.43,1.54,1.64,1.74,1.84,1.95$.

${ }^{6} \mathrm{G}_{7}-{ }^{6} \mathrm{H}_{8}(0.03,0.08,0.13,0.18,0.23,0.33), 1.00,1.05,1.10,1.15,1.20,1.26,1.31,1.36,1.41,1.46,1.51,1.56,1.61$, 1.66 .

${ }^{6} \mathrm{H}_{3}-6 \mathrm{H}^{\prime}{ }_{3}(0.00), 0.29$.

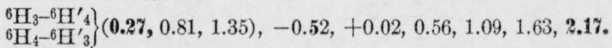

${ }^{6} \mathrm{H}_{4}{ }^{-6} \mathrm{H}^{\prime}{ }_{4}(0.00), 0.82$.

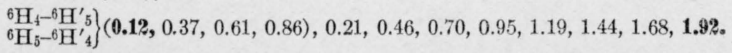

${ }^{6} \mathrm{H}_{5}-6 \mathrm{H}^{\prime}{ }_{5}(0.00), 1.07$.

$\left.{ }^{{ }^{6} \mathrm{H}_{5} \mathrm{H}_{6}{ }^{-6} \mathrm{H}^{\prime} \mathrm{H}_{6}^{\prime}}\right\}$ (

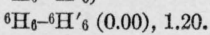

$\left.{ }^{{ }^{6} \mathrm{H}_{6}-\mathrm{H}_{7}-\mathrm{H}^{6} \mathrm{H}^{\prime}{ }_{6}}{ }_{6}\right\}(0.04,0.12,0.20,0.28,0.36,0.43), 0.84,0.92,1.00,1.08,1.16,1.24,1.32,1.40,1.48,1.56,1.64,1.72$.

${ }^{6} \mathrm{H}_{7}-{ }^{6} \mathrm{H}^{\prime}{ }_{7}(0.00), 1.28$.

${ }^{8} \mathrm{H}_{7}-6 \mathrm{H}^{\prime}{ }_{8}(0.03,0.08,0.13,0.18,0.23,0.28,0.33), 1.00,1.05,1.10,1.15,1.20,1.26,1.31,1.36,1.41,1.46,1.51,1.56$,

$\left.{ }^{6} \mathrm{H}_{8}-{ }^{6} \mathrm{H}^{\prime}{ }_{7}\right\}^{1.61,1.6 \%}$.

${ }^{6} \mathrm{H}_{8}-{ }^{6} \mathrm{H}_{8}^{\prime}(0.00), 1.33$.

${ }^{6} \mathrm{H}_{3}-6 \mathrm{I}_{4}(0.08,0.24,0.40), 0.05,0.21,0.36,0.52,0.68,0.84$.

${ }^{6} \mathrm{H}_{4}-6 \mathrm{I}_{4}(0.19,0.57,0.95, \mathbf{1 . 3 3}),-0.51,-0.13,+0.25, \mathbf{0 . 6 3}, 1.01,1.40,1.78$.

${ }^{6} \mathrm{H}_{4}-{ }^{6} \mathrm{I}_{5}(0.00,0.00,0.01,0.01), 0.83,0.83,0.84,0.84,0.84,0.84,0.85,0.85$.

${ }^{6} \mathrm{H}_{5}-6 \mathrm{I}_{4}(\mathbf{0 . 3 1}, 0.94,1.56,2.19),-1.12,-0.49,+0.13,0.76,1.38,2.01,2.63,3.26$.

${ }^{6} \mathrm{H}_{5}-\mathrm{I}_{5}(0.12,0.36,0.61,0.85,1.09),-0.02,+0.22,0.46,0.71,0.95,1.19,1.43,1.67,1.92$.

${ }^{6} \mathrm{H}_{5}{ }^{-6} \mathrm{I}_{6}(\theta .02,0.05,0.09,0.12,0.16), 0.87,0.91,0.95,0.98,1.02,1.05,1.09,1.12,1.16,1.20$.

${ }^{6} \mathrm{H}_{0}-6 \mathrm{I}_{5}(\mathbf{0 . 1 9}, 0.56,0.94,1.31,1.68),-0.48,-0.11,+0.27,0.64,1.02,1.39,1.76,2.14,2.51$, 2.89.

${ }^{6} \mathrm{H}_{6}-{ }^{6} \mathrm{I}_{6}(0.08,0.25,0.42,0.59,0.76,0.92), 0.28,0.45,0.61,0.78,0.95,1.12,1.29,1.45,1.62,1.79,1.96$.

${ }^{6} \mathrm{H}^{6-6} \mathrm{I}_{7}(0.02,0.07,0.11,0.15,0.20,0.24), 0.92,0.96,1.00,1.05,1.09,1.14,1.18,1.22,1.27,1.31,1.35,1.40$.

${ }^{6} \mathrm{H}_{7}-6 \mathrm{I}_{6}(0.12,0.37,0.62,0.86,1.11,1.36),-0.07,+0.17,0.42,0.66,0.91,1.16,1.41,1.65,1.90,2.15,2.39$, 2.64.

${ }^{6} \mathrm{H}_{7}-{ }^{6} \mathrm{I}_{7}(0.06,0.18,0.31,0.43,0.55,0.68,0.80), 0.48,0.60,0.73,0.85,0.97,1.10,1.22,1.34,1.46,1.59,1.71,1.83,1.96$. 
TABLE 3.-Theoretical Zeeman effects (sextet system)-Continued

${ }^{6} \mathrm{H}_{7}-{ }^{6} \mathrm{I}_{8}(0.02,0.06,0.11,0.15,0.19,0.24,0.28), 0.96,1.00,1.05,1.09,1.13,1.17,1.22,1.26,1.30,1.35,1.39,1.43$, $1.47,1.52$.

${ }^{6} \mathrm{H}_{8}{ }^{-} \mathrm{C}_{7}(0.09,0.26,0.44,0.61,0.78,0.96,1.13), 0.20,0.37,0.55,0.72,0.90,1.07,1.25,1.42,1.59,1.77,1.94,2.12$, 2.29, 2.47.

${ }^{6} \mathrm{H}_{8}{ }^{-} \mathrm{I}_{8}(0.05,0.14,0.23,0.32,0.42,0.52,0.61,0.71), 0.63,0.72,0.81,0.91,1.00,1.10,1.19,1.29,1.38,1.47,1.57$, $1.66,1.76,1.85,1.94$.

${ }^{6} \mathrm{H}_{8}{ }^{-6} \mathrm{I}_{9}(0.02,0.06,0.10,0.14,0.18,0.21,0.25,0.29), 1.00,1.04,1.08,1.12,1.16,1.20,1.23,1.27,1.31,1.35,1.39$, $1.43,1.47,1.51,1.55,1.59$.

${ }^{6} \mathrm{I}_{4}-{ }^{6} \mathrm{I}_{4}^{\prime}(0.00), 0.44$.

$\left.{ }^{6} \mathrm{I}_{4} \mathrm{I}_{5}-6 \mathrm{I}^{\prime} \mathrm{I}_{4}^{\prime}{ }_{4}\right\}(0.19,0.58,0.96,1.34),-0.52,-0.13,+0.25,0.64,1.02,1.40,1.79,2.1 \%$.

${ }^{0} \mathrm{I}_{5}-6 \mathrm{I}^{\prime}{ }_{5}(0.00), 0.83$.

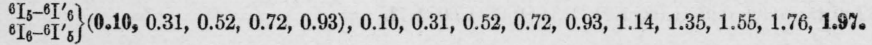

${ }^{6} \mathrm{I}_{6}-6 \mathrm{I}^{\prime}{ }_{6}(0.00), 1.04$.

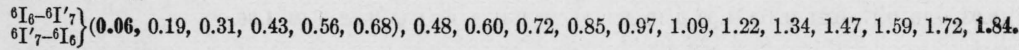

${ }^{6} \mathrm{I}_{7}-6 \mathrm{I}_{7}{ }_{7}(0.00), 1.16$.

$\left.{ }^{6} \mathrm{I}_{7-6} \mathrm{I}^{\prime}{ }_{8}\right)(0.04,0.12,0.20,0.27,0.36,0.44,0.52), 0.72,0.80,0.88,0.96,1.04,1.12,1.20,1.28,1.36,1.44,1.52,1.60$, $\left.{ }^{6} \mathrm{I}_{8}-6 \mathrm{I}_{7}^{\prime}\right\}^{0} \quad 1.68,1.76$.

${ }^{6} \mathrm{I}_{8}-6 \mathrm{I}_{8}^{\prime}(0.00), 1.24$.

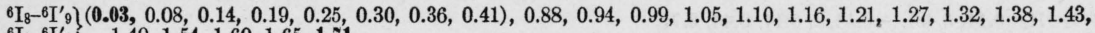

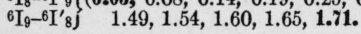

${ }^{6} \mathrm{I}_{9}-6 \mathrm{I}^{\prime}{ }_{0}(0.00), 1.28$. 
TABLE 4.-Theoretical Zeeman effects (octet system)

[Landé $g$ values]

\begin{tabular}{|c|c|c|c|c|c|c|c|c|c|c|c|c|c|c|c|c|c|c|c|c|}
\hline$i^{j}$ & 1 & 2 & 3 & 4 & 5 & 6 & 7 & 8 & 9 & 10 & 1 & 2 & 3 & 4 & 5 & 6 & 7 & 8 & 9 & 10 \\
\hline $\mathrm{s}$ & & & & $\frac{1296}{63}$ & & & & & & & & & & 2.000 & & & & & & \\
\hline $\mathrm{P}$ & - & & $\frac{80}{35}$ & $\frac{129}{63}$ & $\frac{176}{89}$ & & & & & & & & 2. 286 & 1.937 & 1.778 & & & & & \\
\hline $\mathrm{D}$ & & $\frac{4}{15} \frac{2}{5}$ & $\frac{72}{35}$ & $\frac{214}{63}$ & $\frac{168}{89}$ & $\frac{234}{143}$ & & & & & & 2.800 & 2. 057 & 1.809 & 1.697 & 1.636 & & & & \\
\hline $\mathrm{F}$ & $\frac{12}{3}$ & $\frac{30}{15}$ & $\frac{60}{35}$ & $\frac{102}{63}$ & $\frac{156}{99}$ & $\frac{222}{143}$ & $\frac{300}{195}$ & & & & 4. 000 & 2.000 & 1. 714 & 1.619 & 1.576 & 1. 552 & 1.538 & & & \\
\hline$G$ & $-\frac{4}{3}$ & $\frac{14}{15}$ & $\frac{44}{35}$ & $\frac{86}{63}$ & $\frac{140}{98}$ & $\frac{206}{143}$ & $\frac{284}{195}$ & $\frac{374}{235}$ & & & -1.333 & 0.933 & 1. 257 & 1. 365 & 1.414 & 1. 441 & 1. 456 & 1. 467 & & \\
\hline H & & $-\frac{6}{15}$ & $\frac{24}{35}$ & $\frac{66}{63}$ & $\frac{120}{99}$ & $\frac{186}{143}$ & $\frac{264}{19}$ & $\frac{354}{255}$ & $\frac{4}{3} \frac{56}{3}$ & & & -0.400 & 0.686 & 1.048 & 1.212 & 1. 301 & 1. 354 & 1. 388 & 1. 412 & \\
\hline I & & & $\frac{0}{35}$ & $\frac{4 \frac{3}{6}}{6}$ & $\frac{96}{98}$ & $\frac{162}{14 \frac{2}{3}}$ & $\frac{240}{19} \frac{0}{5}$ & $\frac{330}{25}$ & $\frac{432}{323}$ & $\frac{848}{398}$ & & & 0.000 & 0.667 & 0.970 & 1. 133 & 1. 231 & 1. 294 & 1. 337 & 1. 368 \\
\hline
\end{tabular}


TABLE 4.-Theoretical Zeeman effects (octet system)-Continued

${ }^{8} \mathrm{~S}_{4}-8 \mathrm{~S}_{4}^{\prime}(0.00), 2.00$.

${ }^{8} \mathrm{~S}_{4}{ }^{-8} \mathrm{P}_{3}(0.14,0.43,0.71), 1.29,1.57,1.86,2.14,2.43,2.72$.

${ }^{8} \mathrm{~S}_{4}{ }^{-8} \mathrm{P}_{4}(0.03,0.10,0.16,0.22), 1.78,1.84,1.90,1.97,2.03,2.10,2.16$.

${ }^{8} \mathrm{~S}_{4}{ }^{-8} \mathrm{P}_{5}(0.11,0.33,0.56,0.78), 1.00,1.22,1.44,1.67,1.89,2.11,2.33,2.55$.

${ }^{8} \mathrm{~S}_{4}{ }^{-8} \mathrm{D}_{3}^{\prime}{ }_{3}(0.03,0.09,0.14), 1.86,1.92,1.97,2.03,2.09,2.15$.

${ }^{8} \mathrm{~S}_{4^{-8}}{ }^{8} \mathrm{D}_{4}^{\prime}(0.10,0.29,0.48,0.67), 1.33,1.52,1.72,1.31,2.10,2.29,2.48$.

${ }^{8} \mathrm{~S}_{4}{ }^{8} \mathrm{DD}_{5}^{\prime}(0.15,0.45,0.76,1.06), 0.64,0.94,1.24,1.55,1.85,2.15,2.46,2.76$.

${ }^{8} \mathrm{~S}_{4}-8 \mathrm{~F}_{3}(\mathbf{0 . 1 4}, 0.43,0.72), 1.29,1.57,1.86,2.14,2.43,2.72$.

${ }^{8} \mathrm{~S}_{4}{ }^{8} \mathrm{~F}_{4}(0.19,0.57,0.95,1.33), 0.67,1.05,1.43,1.81,2.19,2.57,2.95$.

${ }^{8} \mathrm{~S}_{4}-8 \mathrm{~F}_{5}(\mathbf{0 . 2 1}, 0.64,1.06,1.48), \mathbf{0 . 0 9 ,} 0.52,0.94,1.36,1.79,2.21,2.64,3.06$.

${ }^{8} \mathrm{P}_{3}-8 \mathrm{P}_{3}^{\prime}(0.00), 2.28$.

$\left.{ }_{8}^{8 \mathrm{P}_{3} \mathrm{P}_{4}^{-8} \mathrm{P}^{\prime} \mathrm{P}^{\prime}{ }_{3}}\right\}(0.17,0.52,0.87), 1.06,1.41,1.76,2.11,2.46,2.81$.

${ }^{8} \mathrm{P}_{4}-8 \mathrm{P}_{4}^{\prime}(0.00), 1.94$.

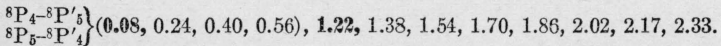

${ }^{8} \mathrm{P}_{5}-{ }^{8} \mathrm{P}_{5}^{\prime}(0.00), 1.78$.

${ }^{8} \mathrm{P}_{3}-{ }^{-8} \mathrm{D}_{2}(\mathbf{0 . 2 6}, 0.77), \mathbf{1 . 5 1}, 2.03,2.54,3.06$.

${ }^{8} \mathrm{P}_{3}-8 \mathrm{D}_{3}(0.11,0.34,0.57), 1.71,1.94,2.17,2.40,2.63$.

${ }^{8} \mathrm{P}_{3}-8 \mathrm{D}_{4}(0.24,0.71,1.19), 0.62,1.09,1.57,2.05,2.53,3.00$.

${ }^{8} \mathrm{P}_{4}{ }^{-8} \mathrm{D}_{3}(0.06,0.18,0.30), 1.63,1.76,1.88,2.00,2.12,2.24$.

${ }^{8} \mathrm{P}_{4}{ }^{-8} \mathrm{D}_{4}(0.06,0.19,0.32,0.44), 1.49,1.62,1.75,1.87,2.00,2.12,2.25$.

${ }^{8} \mathrm{P}_{4}{ }^{-8} \mathrm{D}_{5}(0.12,0.36,0.60,0.84), 0.86,1.10,1.34,1.58,1.81,2.05,2.29,2.53$.

${ }^{8} \mathrm{P}_{5}{ }^{8} \mathrm{D}_{4}(\mathbf{0 . 0 2}, 0.05,0.08,0.11), 1.67,1.70,1.73,1.76,1.79,1.82,1.86,1.89$.

${ }^{8} \mathrm{P}_{5}-{ }^{8} \mathrm{D}_{5}(0.04,0.12,0.20,0.28,0.36), 1.41,1.49,1.58,1.66,1.74,1.82,1.90,1.98,2.06$.

${ }^{8} \mathrm{P}_{5}{ }^{-8} \mathrm{D}_{6}(0.07,0.21,0.35,0.49,0.64), 1.00,1.14,1.28,1.42,1.57,1.71,1.85,1.99,2.13,2.27$.

${ }^{8} \mathrm{P}_{3}-{ }^{8} \mathrm{~F}_{2}^{\prime}$ (0.14, 0.43), 1.86, 2.14, 2.43, 2.7\%.

${ }^{8} \mathrm{P}_{3}-8 \mathrm{~F}^{\prime}{ }_{3}(0.29,0.86,1.43), 0.86,1.43,2.00,2.57,3.14$.

${ }^{8} \mathrm{P}_{3}-8 \mathrm{~F}^{\prime}{ }_{4}(0.33,1.00,1.67),-0.05,+0.62,1.28,1.95,2.62,3.29$.

${ }^{8} \mathrm{P}_{4}{ }^{-8} \mathrm{~F}^{\prime}{ }_{3}(0.11,0.33,0.56), 1.38,1.60,1.83,2.05,2.27,2.49$.

${ }^{8} \mathrm{P}_{4}-8 \mathrm{~F}^{\prime}{ }_{4}(0.16,0.48,0.79,1.11), 0.82,1.14,1.46,1.78,2.09,2.41,2.73$.

${ }^{8} \mathrm{P}_{4}-8 \mathrm{~F}^{\prime}{ }_{5}(0.18,0.54,0.90,1.26), 0.31,0.67,1.03,1.39,1.75,2.11,2.47,2.83$.

${ }^{8} \mathrm{P}_{5}{ }^{-8} \mathrm{~F}_{4}^{\prime}(\theta .08,0.24,0.40,0.56), 1.22,1.38,1.54,1.70,1.86,2.02,2.18,2.33$.

${ }^{8} \mathrm{P}_{5}{ }^{8} \mathrm{~F}_{5}{ }_{5}(0.10,0.30,0.50,0.71,0.91), 0.87,1.07,1.27,1.47,1.68,1.88,2.08,2.28,2.48$.

${ }^{8} \mathrm{P}_{5}{ }^{-8} \mathrm{~F}^{\prime}{ }_{6}(0.11,0.34,0.56,0.79,1.02), 0.54,0.76,0.99,1.22,1.44,1.67,1.89,2.12,2.34,2.57$.

${ }^{8} \mathrm{P}_{3}-{ }^{8} \mathrm{G}_{2}(0.68,2.03), 0.26,1.61,2.96,4.31$.

${ }^{8} \mathrm{P}_{3}{ }^{-8} \mathrm{G}_{3}(0.51,1.54,2.57),-0.29,+0.74,1.77,2.80,3.83$.

${ }^{8} \mathrm{P}_{3}{ }^{-8} \mathrm{G}_{4}(0.46,1.38,2.30),-0.94,-0.02,+0.90,1.82,2.74,3.66$.

${ }^{8} \mathrm{P}_{4}{ }^{-8} \mathrm{G}_{3}(0.34,1.02,1.70), 0.24,0.92,1.60,2.27,2.95,3.63$.

${ }^{8} \mathrm{P}_{4}{ }^{-8} \mathrm{G}_{4}(0.29,0.86,1.43,2.00),-0.06,+0.51,1.08,1.65,2.22,2.79,3.36$.

${ }^{8} \mathrm{P}_{4^{-8}} \mathrm{G}_{5}(0.26,0.78,1.30,1.83),-0.41,+0.11,0.63,1.15,1.68,2.20,2.72,3.24$.

${ }^{8} \mathrm{P}_{5}{ }^{-8} \mathrm{G}_{4}(\mathbf{0 . 2 1}, 0.62,1.03,1.44), 0.33,0.75,1.16,1.57,1.98,2.39,2.80,3.22$.

${ }^{8} \mathrm{P}_{5}{ }^{-8} \mathrm{G}_{5}(0.18,0.55,0.91,1.27,1.64), 0.14,0.50,0.87,1.23,1.60,1.96,2.32,2.69,3.05$.

${ }^{8} \mathrm{P}_{5}{ }^{-8} \mathrm{G}_{0}(0.17,0.51,0.84,1.18,1.52),-0.08,+0.26,0.60,0.94,1.27,1.61,1.95,2.28,2.62,2.96$.

${ }^{8} \mathrm{D}_{2}{ }^{-8} \mathrm{D}_{2}^{\prime}(0.00), 2.80$.

$\left.{ }_{8 D_{2}-8 D_{3}^{-8} D_{3}^{\prime}{ }_{2} D_{2}}^{8 D_{2}}\right\}(0.37,1.11), 0.94,1.68,2.43,3.17$.

${ }^{8} \mathrm{D}_{3}{ }^{-8} \mathrm{D}_{3}^{\prime}(0.00), 2.06$.

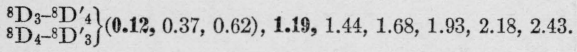

${ }^{8} \mathrm{D}_{4}{ }^{-8} \mathrm{D}_{4}^{\prime}(0.00), 1,81$.

$\left.{ }^{8} \mathrm{D}_{4^{-}-8 \mathrm{D}^{-8} \mathrm{D}_{5}^{\prime}}^{8} \mathrm{D}_{4}^{\prime}\right\}(0.06,0.17,0.28,0.39), 1.30,1.41,1.53 .1 .64,1.75,1.86,1.98,2.09$.

${ }^{8} \mathrm{D}_{5}-8 \mathrm{D}_{5}^{\prime}(0.00), 1.70$.

$\left.{ }^{8} \mathrm{D}_{5} \mathrm{D}_{6}^{-8} \mathrm{~B}^{-8} \mathrm{D}_{6}^{\prime}{ }_{5}^{\prime}\right\}(0.03,0.09,0.15,0.21,0.27), 1.36,1.42,1.48,1.54,1.61,1.67,1.73,1.79,1.85,1.91$.

${ }^{8} \mathrm{D}_{6}{ }^{-8} \mathrm{D}_{6}^{\prime}(0.00), 1.63$.

${ }^{8} \mathrm{D}_{2}-8 \mathrm{~F}_{1}(0.60), 2.20,3.40$.

${ }^{8} \mathrm{D}_{2}-8 \mathrm{~F}_{2}(0.40,1.20), 1.60,2.40,3.20$.

${ }^{8} \mathrm{D}_{2}-8 \mathrm{~F}_{3}(0.54,1.63), 0.09,1.17,2.26,3.34$.

${ }^{8} \mathrm{D}_{3}-8 \mathrm{~F}_{2}(0.03,0.09), 1.97,2.03,2.08,2.14$,

${ }^{8} \mathrm{D}_{3}{ }^{-8} \mathrm{~F}_{3}(0.17,0.51,0.86), 1.20,1.54,1.88,2.23,2.57$.

${ }^{8} \mathrm{D}_{3}-8 \mathrm{~F}_{4}(0.22,0.66,1.10), 0.52,0.96,1.40,1.84,2.27,2.72$. 
TABLE 4.-Theoretical Zeeman effects (octet system)-Continued

${ }^{8} \mathrm{D}_{4}-8 \mathrm{~F}_{3}(0.05,0.14,0.24), 1.57,1.66,1.76,1.85,1.95,2.05$.

${ }^{8} \mathrm{D}_{4}{ }^{8} \mathrm{~F}_{4}(0.09,0.28,0.48,0.67), 1.14,1.33,1.52,1.71,1.90,2.09,2.28$.

${ }^{8} \mathrm{D}_{4}-8 \mathrm{~F}_{5}(0.12,0.35,0.58,0.82), 0.76,0.99,1.22,1.46,1.69,1.93,2.16,2.39$.

${ }^{8} \mathrm{D}_{5}-8 \mathrm{~F}_{4}(0.04,0.12,0.19,0.27), 1.42,1.50,1.58,1.66,1.73,1.81,1.89,1.97$.

${ }^{8} \mathrm{D}_{5}-8 \mathrm{~F}_{5}(0,06.0 .18,0.30,0.42,0.54), 1.15,1.27,1.39,1.51,1.64,1.76,1.88,2.00,2.12$.

${ }^{8} \mathrm{D}_{5}-8 \mathrm{~F}_{6}(0.07,0.22,0.36,0.51,0.65), 0.90,1.05,1.19,1.33,1.48,1.62,1.77,1.91,2.06,2.20$.

${ }^{8} \mathrm{D}_{6}{ }^{-8} \mathrm{~F}_{5}(0.03,0.09,0.15,0.21,0.27), 1.36,1.42,1.49,1.55,1.61,1.67,1.73,1.79,1.85,1.91$.

${ }^{8} \mathrm{D}_{6}{ }^{-8} \mathrm{~F}_{6}(0.04,0.13,0.21,0.29,0.38,0.46), 1.18,1.26,1.34,1.43,1.51,1.60,1.68,1.76,1.85,1.93,2.01$.

${ }^{8} \mathrm{D}_{0}{ }^{8} \mathrm{~F}_{7}(0.05,0.15,0.24,0.34,0.44,0.54), 1.00,1.10,1.20,1.29,1.39,1.49,1.59,1.68,1.78,1.88,1.98,2.08$.

${ }^{8} \mathrm{D}_{2}-8 \mathrm{G}_{1}^{\prime}{ }_{1}(2.07), 0.73,4.8 \%$.

${ }^{8} \mathrm{D}_{2}{ }^{-8} \mathrm{G}_{2}^{\prime}(0.93,2.80), 0.00,1.8 \%, 3.74$.

${ }^{8} \mathrm{D}_{2}-{ }^{8} \mathrm{G}^{\prime}{ }_{3}(0.77,2.31),-1.06,+0.49,2.03,3.57$.

${ }^{8} \mathrm{D}_{3}-8 \mathrm{G}^{\prime}{ }_{3}(0.56,1.69), 0.37,1.50,2.62,3.74$.

${ }^{8} \mathrm{D}_{3}-8 \mathrm{G}^{\prime}{ }_{3}(0.40,1.20,2.00), 0.06,0.86,1.66,2.46,3.26$.

${ }^{8} \mathrm{D}_{3}-8 \mathrm{G}^{\prime}{ }_{4}(0.35,1.04,1.73),-0.36,+0.33,1.02,1.71,2.40,3.10$.

${ }^{8} \mathrm{D}_{4}{ }^{-8} \mathrm{G}_{3}{ }_{3}(0.28,0.83,1.38), 0.43,0.98,1.53,2.09,2.64,3.19$.

${ }^{8} \mathrm{D}_{4}{ }^{-8} \mathrm{G}_{4}{ }_{4}(0.22,0.67,1.11,1.56), 0.25,0.70,1.14,1.58,2.03,2.48,2.92$.

${ }^{8} \mathrm{D}_{4}-8 \mathrm{G}^{\prime}{ }_{5}(0.20,0.59,0.99,1.38), 0.03,0.43,0.82,1.22,1.61,2.01,2.40,2.80$.

${ }^{8} \mathrm{D}_{5}-8 \mathrm{G}_{4}{ }_{4}(0.17,0.50,0.83,1.16), 0.54,0.87,1.20,1.53,1.86,2.19,2.53,2.86$.

${ }^{8} \mathrm{D}_{5}{ }^{-8} \mathrm{G}^{\prime}{ }_{5}(0.14,0.42,0.71,0.99,1.27), 0.42,0.71,0.99,1.27,1.56,1.84,2.12,2.40,2.69$.

${ }^{8} \mathrm{D}_{5}{ }^{-8} \mathrm{G}_{6}{ }_{6}(0.13,0.38,0.64,0.90,1.15), 0.29,0.54,0.80,1.06,1.31,1.57,1.82,2.08,2.33,2.59$.

${ }^{8} \mathrm{D}_{6}{ }^{-8} \mathrm{G}_{5}^{\prime}(0.11,0.33,0.56,0.78,1.00), 0.64,0.86,1.08,1.30,1.53,1.75,1.97,2.19,2.42,2.64$.

${ }^{8} \mathrm{D}_{6}-{ }^{8} \mathrm{G}^{\prime}{ }_{6}(0.10,0.29,0.49,0.68,0.88,1.08), 0.56,0.76,0.95,1.15,1.34,1.54,1.73,1.93,2.13,2.32,2.52$.

${ }^{8} \mathrm{D}_{6}{ }^{-8} \mathrm{G}^{\prime}{ }_{7}(0.09,0.27,0.45,0.63 .0 .81 \cdot 0.99), 0.47,0.65,0.83,1.01,1.19,1.37,1.55,1.73,1.91,2.09,2.27,2.45$.

${ }^{8} \mathrm{~F}_{1}-8 \mathrm{~F}^{\prime}{ }_{1}(0.00), 4.00$.

$\left.\begin{array}{l}{ }^{8} \mathrm{~F}_{1}-8 \mathrm{~F}^{\prime}{ }_{2} \\ { }^{8} \mathrm{~F}_{2}-8 \mathrm{~F}_{1}^{\prime}\end{array}\right\}(1.00), \mathbf{1 . 0 0}, 3.00$.

${ }^{8} \mathrm{~F}_{2}-8 \mathrm{~F}_{2}^{\prime}(0.00), 2.00$.

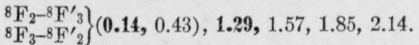

${ }^{8} \mathrm{~F}_{3}-8 \mathrm{~F}^{\prime}{ }_{3}(0.00), 1.71$.

$\left.\begin{array}{l}{ }^{8} \mathrm{~F}_{3}-8 \mathrm{~F}^{\prime}{ }^{8} \mathrm{~F}_{4}^{-8} \mathrm{~F}_{3}^{\prime} \\ { }^{8}\end{array}\right\}(0.05,0.14,0.24), 1.38,1.48,1.57,1.67,1.76,1.86$.

$8 \mathrm{~F}_{4}-8 \mathrm{~F}^{\prime}{ }_{4}(0.00), 1.62$.

$\left.{ }^{8} \mathrm{~F}_{4_{5}^{-8}-8 \mathrm{~F}^{\prime}{ }_{5}{ }_{4}^{8}}^{8}\right\}(0.02,0.06,0.11,0.15), 1.42,1.47,1.51,1.55,1.60,1.64,1.68,1.73$

${ }^{8} \mathrm{~F}_{5}-8 \mathrm{~F}^{\prime}{ }_{5}(0.00), 1.57$.

$\left.{ }_{8}^{8 \mathrm{~F}_{5}-8 \mathrm{~F}_{6}{ }^{8} \mathrm{~F}_{6} \mathrm{~F}_{5}}\right\}(0.01,0.03,0.06,0.08,0.10), 1.45,1.47,1.49,1.52,1.54,1.56,1.59,1.61,1.63,1.66$.

${ }^{8} \mathrm{~F}_{6}-8 \mathrm{~F}^{\prime}{ }_{6}(0.00), 1.55$.

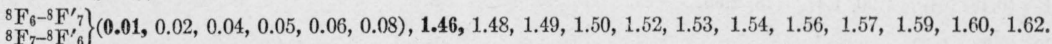

${ }^{8} \mathrm{~F}_{7}-8 \mathrm{~F}^{\prime}{ }_{7}(0.00), 1.54$.

${ }^{8} \mathrm{~F}_{1}-8 \mathrm{G}_{1}(2.67), 1.33$.

${ }^{8} \mathrm{~F}_{1}-8 \mathrm{G}_{2}(1.53),-0.60,+2.47$.

${ }^{8} \mathrm{~F}_{2}-8 \mathrm{G}_{1}(1.67), 0.33,3.6 \%$.

${ }^{8} \mathrm{~F}_{2}-8 \mathrm{G}_{2}(0.53,1.60), 0.40,1.47,2.53$.

${ }^{8} \mathrm{~F}_{2}-{ }^{-8} \mathrm{G}_{3}(0.37,1.11), 0.14,0.89,1.63,2.37$.

${ }^{8} \mathrm{~F}_{3}-8 \mathrm{G}_{2}(0.39,1.17), 0.54,1.33,2.10,2.38$.

${ }^{8} \mathrm{~F}_{3}-8 \mathrm{G}_{3}(0.23,0.69,1.14), 0.57,1.03,1.49,1.94,2.40$.

${ }^{8} \mathrm{~F}_{3}-{ }^{-8} \mathrm{G}_{4}(0.17,0.52,0.87), 0.49,0.84,1.19,1.54,1.89,2.24$.

${ }^{8} \mathrm{~F}_{4}{ }^{-8} \mathrm{G}_{3}(0.18,0.54,0.90), 0.71,1.08,1.44,1.80,2.16,2.52$.

${ }^{8} \mathrm{~F}_{4}{ }^{-8} \mathrm{G}_{4}(0.13,0.38,0.63,0.89), 0.73,0.98,1.24,1.49,1.75,2.00,2.25$.

${ }^{8} \mathrm{~F}_{4^{-8}} \mathrm{G}_{5}(0.10,0.31,0.51,0.72), 0.70,0.90,1.11,1.31,1.52,1.72,1.92,2.13$.

${ }^{8} \mathrm{~F}_{5}-8 \mathrm{G}_{4}(\mathbf{0 . 1 1}, 0.32,0.53,0.74), 0.84,1.05,1.26,1.47,1.68,1.89,2.11$, 2.32.

${ }^{8} \mathrm{~F}_{5}-8 \mathrm{G}_{5}(0.08,0.24,0.40,0.57,0.73), 0.85,1.01,1.17,1.33,1.49,1.66,1.82,1.98,2.14$.

${ }^{8} \mathrm{~F}_{5}-8 \mathrm{G}_{6}(0.07,0.20,0.34,0.47,0.61), 0.83,0.97,1.10,1.24,1.37,1.51,1.64,1.77,1.91,2.05$.

${ }_{8}^{8} \mathrm{~F}_{6}-8 \mathrm{G}_{5}(0.07,0.21,0.35,0.48,0.62), 0.93,1.07,1.21,1.35,1.48,1.62,1.76,1.90,2.04,2.18$.

${ }^{8} \mathrm{~F}_{5}-8 \mathrm{G}_{6}(0.06,0.17,0.28,0.39,0.50,0.62), 0.94,1.05,1.16,1.27,1.38,1.50,1.61,1.72,1.83,1.94,2.05$.

${ }^{8} \mathrm{~F}_{6}{ }^{-8} \mathrm{G}_{7}(0.05,0.14,0.24 .0 .34,0.43,0.53), 0.93,1.02,1.12,1.22,1.31,1.41,1.50,1.60,1.70,1.79,1.89,1.99$.

${ }^{8} \mathrm{~F}_{7}-8 \mathrm{G}_{6}(\mathbf{0 . 0 5}, 0.15,0.24,0.34,0.44,0.54), 1.00,1.10,1.20,1.29,1.39,1.49,1.58,1.68,1.78,1.88,1.98,2.08$.

${ }^{8} \mathrm{~F}_{7}-{ }^{8} \mathrm{G}_{7}(0.04,0.12,0.20,0.29,0.37,0.45,0.53), 1.00,1.09,1.17,1.25,1.33,1.42,1.50,1.58,1.66,1.75,1.83,1.91$, 1.99.

${ }^{8} \mathrm{~F}_{7}{ }^{-8} \mathrm{G}_{8}(\mathbf{0 . 0 4}, 0.11,0.18,0.25,0.32,0.39,0.47), 1.00,1.07,1.14,1.22,1.29,1.36,1.43,1.50,1.57,1.65,1.72,1.79$, $1.86,1.93$. 
TABLE 4.-Theoretical Zeeman effects (octet system)-Continued

${ }^{8} \mathrm{G}_{1}-{ }^{8} \mathrm{G}_{1}^{\prime}(0.00), 1.33$.

$\left.\begin{array}{l}{ }^{8} \mathrm{G}_{1}-8 \mathrm{G}^{\prime}{ }_{2} \\ { }^{8} \mathrm{G}_{2}-8 \mathrm{G}^{\prime}{ }_{1}\end{array}\right\}(1.13),-0.20,+2.07$.

${ }^{8} \mathrm{G}_{2}-8 \mathrm{G}^{\prime}{ }_{2}(0.00), 0.93$.

$\left.{ }^{8} \mathrm{G}_{2^{-8}-8} \mathrm{G}^{\prime}{ }_{3}{ }_{3}^{8} \mathrm{G}_{2}{ }_{2}\right\}(0.16,0.48), 0.77,1.09,1.42,1.74$.

${ }^{8} \mathrm{G}_{3}-8 \mathrm{G}^{\prime}{ }_{3}(0.00), 1.26$.

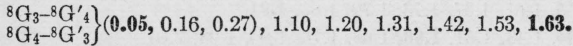

${ }^{8} \mathrm{G}_{4}-8 \mathrm{G}^{\prime}{ }_{4}(0.00), 1.37$.

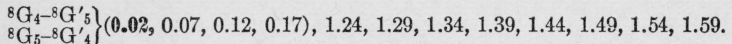

${ }^{8} \mathrm{G}_{5}^{-8} \mathrm{G}_{5}^{\prime}(0.00), 1.41$.

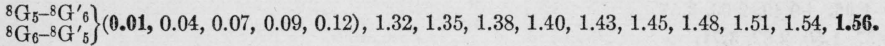

${ }^{8} \mathrm{G}_{6}-8 \mathrm{G}^{\prime}{ }_{6}(0.00), 1.44$.

$\left.{ }^{8} \mathrm{G}_{6-8}^{8} \mathrm{G}^{\prime} \mathrm{G}_{7}{ }^{8} \mathrm{G}_{6}^{\prime}\right\}(0.01,0.02,0.04,0.06,0.07,0.09), 1.37,1.39,1.40,1.42,1.43,1.45,1.46,1.48,1.50,1.51,1.53,1.54$.

${ }^{8} \mathrm{G}_{7-8} \mathrm{G}^{\prime}{ }_{7}(0.00), 1.46$

$\left.{ }^{8} \mathrm{G}_{7-8} \mathrm{G}^{\prime}{ }_{8}\right)(0.01,0.02,0.03,0.04,0.05,0.06,0.07), 1.40,1.41,1.42,1.43,1.44,1.45,1.46,1.47,1.48,1.49,1.50,1.51$, $\left.{ }^{8} \mathrm{G}_{8}-^{8} \mathrm{G}^{\prime} 7\right\} 1.52, \mathbf{1 . 5 3}$.

${ }^{8} \mathrm{G}_{8}-{ }^{8} \mathrm{G}_{8}^{\prime}(0.00), 1.47$.

TABLE 5.-Theoretical Zeeman effects (doublet-quartet intersystem)

${ }^{2} \mathrm{~S}_{1}-4 \mathrm{~S}_{2}{ }_{2}(0.00), 2.00$.

${ }^{2} \mathrm{~S}_{1}{ }^{-4} \mathrm{P}_{1}(0.33), 2.33$.

${ }^{2} \mathrm{~S}_{1}-4 \mathrm{P}_{2}(0.13), 1.60,1.87$.

${ }^{2} \mathrm{~S}_{1}-4 \mathrm{D}^{\prime}{ }_{1}(1.00), 1.00$.

${ }^{2} \mathrm{~S}_{1}{ }^{4} \mathrm{D}_{2}^{\prime}(0.40), 0.80,1.60$.

${ }^{2} \mathrm{~S}_{1}-4 \mathrm{~F}_{2}(0.80),-0.40,+1.20$.

${ }^{2} \mathrm{P}_{1}-4 \mathrm{~S}_{2}(0.67), 1.33,2.6 \%$.

${ }^{2} \mathrm{P}_{2}-{ }^{4} \mathrm{~S}_{2}(0.33,1.00), 1.00,1.67,2.33$.

${ }^{2} \mathrm{P}_{1}-4 \mathrm{P}_{1}^{\prime}(1.00), 1.67$.

${ }^{2} \mathrm{P}_{1-4} \mathrm{P}_{2}^{\prime}(0.53), 1.20$, 2.27.

${ }^{2} \mathrm{P}_{2}{ }^{-4} \mathrm{P}_{1}^{\prime}{ }_{1}(0.67), 0.67,2.00$.

${ }^{2} \mathrm{P}_{2}-4 \mathrm{P}_{2}^{\prime}{ }_{2}(0.20,0.60), 1.13,1.53,1.93$.

${ }^{2} \mathrm{P}_{2}-4 \mathrm{P}_{3}^{\prime}(\mathbf{0 . 1 3}, 0.40), 1.20,1.47,1.73, \mathbf{2 . 6 0}$.

${ }^{2} \mathrm{P}_{1}-4 \mathrm{D}_{1}$ (0.33), 0.33 .

${ }^{2} \mathrm{P}_{1}-\mathrm{D}_{2}(0.27), 0.93,1.47$.

${ }^{2} \mathrm{P}_{2}-{ }^{4} \mathrm{D}_{1}(0.67), 0.67,2.00$.

${ }^{2} \mathrm{P}_{2}-{ }^{4} \mathrm{D}_{2}(0.07,0.20), 1.13,1.27 .1 .40$.

${ }^{2} \mathrm{P}_{2}-{ }^{4} \mathrm{D}_{3}(0.02,0.06), 1.31,1.35,1.39,1.43$.

${ }^{2} \mathrm{P}_{1}-4 \mathrm{~F}^{\prime}{ }_{2}(0.13), 0.27,0.53$.

${ }^{2} \mathrm{P}_{2}-4 \mathrm{~F}^{\prime}{ }_{2}(0.47,1.40),-0.07,+0.87,1.80$.

${ }^{2} \mathrm{P}_{2}-4 \mathrm{~F}^{\prime}{ }_{3}(0.15,0.46), 0.57,0.88,1.18,1.49$.

${ }^{2} \mathrm{P}_{2}{ }^{-4} \mathrm{G}_{3}(0.38,1.14),-0.57,+0.19,0.95$.

${ }^{2} \mathrm{D}_{2}-4 \mathrm{~S}_{2}{ }_{2}(0.60,1.80), 0.20,1.40,2.60$.

${ }^{2} \mathrm{D}_{3}{ }^{-4} \mathrm{~S}_{2}{ }_{2}(0.40,1.20), 0.00,0.80,1.60,2.40$.

${ }^{2} \mathrm{D}_{2}-4 \mathrm{P}_{1}(0.98), 0.13,1.73$.

${ }^{2} \mathrm{D}_{2}-{ }^{-4} \mathrm{P}_{2}(0.47,1.40), 0.33,1.2 \%, 2.20$.

${ }^{2} \mathrm{D}_{2}{ }^{-4} \mathrm{P}_{3}(0.40,1.20), 0.40,1.20,2.00,2.80$,

${ }^{2} \mathrm{D}_{3}-{ }^{4} \mathrm{P}_{2}(0.27,0.80), 0.40,0.93,1.47,2.00$.

${ }^{2} \mathrm{D}_{3}-{ }^{4} \mathrm{P}_{3}(0.20,0.60,1.00), 0.60,1.00,1.40,1.80,2.20$.

${ }^{2} \mathrm{D}_{2}-{ }^{4} \mathrm{D}_{1}^{\prime}(0.40), 0.40,1.20$.

${ }^{2} \mathrm{D}_{2}{ }^{4} \mathrm{D}^{\prime}{ }_{2}(0.20,0.60), 0.60,1.00,1.40$.

${ }^{2} \mathrm{D}_{2}-4 \mathrm{D}^{\prime}{ }_{3}(0.29,0.86), 0.51,1.08,1.66,2.23$.

${ }^{2} \mathrm{D}_{3}{ }^{4} \mathrm{D}^{\prime}{ }_{2}(0.00), 1.20$.

${ }^{2} \mathrm{D}_{3}{ }^{4} \mathrm{D}^{\prime}{ }_{3}(0.09,0.26,0.43), 0.94,1.11,1.29,1.46,1.63$.

${ }^{2} \mathrm{D}_{3}{ }^{4} \mathrm{D}_{4}^{\prime}(0.11,0.34,0.57), 0.86,1.09,1.31,1.54,1.77,2.00$. 
TABLE 5.-Theoretical Zeeman effects (doublet-quartet intersystem)-Continued

${ }^{2} \mathrm{D}_{2}-4 \mathrm{~F}_{2}(0.20,0.60), 0.20 .0 .60,1.00$.

${ }^{2} \mathrm{D}_{2}{ }^{4} \mathrm{~F}_{3}(0.11,0.34), 0.69,0.91,1.14,1.3 \%$.

${ }^{2} \mathrm{D}_{3}{ }^{-4} \mathrm{~F}_{2}(0.40,1.20), 0.00,0.80,1.60,2.40$.

${ }^{2} \mathrm{D}_{3}{ }^{4} \mathrm{~F}_{3}(0.09,0.26,0.43), 0.77,0.94,1.11,1.28,1.44$.

${ }^{2} \mathrm{D}_{3}-4 \mathrm{~F}_{4}(0.02,0.06,0.10), 1.14,1.18,1.22,1.26,1.29$.

${ }^{2} \mathrm{D}_{2}-4 \mathrm{G}_{3}{ }_{3}(\mathbf{0 . 1 1}, 0.34), 0.23,0.46,0.69,0.91$.

${ }^{2} \mathrm{D}_{3}{ }^{-4} \mathrm{G}^{\prime}{ }_{3}(0.31,0.94,1.57),-0.37,+0.26,0.88,1.51,2.14$.

${ }^{2} \mathrm{D}_{3}{ }^{-4} \mathrm{G}_{4}^{\prime}{ }_{4}(0.11,0.32,0.54), 0.44,0.66,0.88,1.00,1.31,1.52$.

${ }^{2} \mathrm{D}_{3}-4 \mathrm{H}_{4}(0.2 \%, 0.80,1.33),-0.6 \%,-0.13,+0.40,0.93,1.47,2.00$.

${ }^{2} \mathrm{~F}_{3}-{ }^{4} \mathrm{~S}_{2}(0.57,1.72),-0.86,+0.29,1.43,2.57$.

${ }^{2} \mathrm{~F}_{3}-4 \mathrm{P}^{\prime}{ }_{2}(0.44,1.31),-0.46,+0.42,1.30,2.17$.

${ }^{2} \mathrm{~F}_{3}-4 \mathrm{P}_{3}^{\prime}(0.37,1.11,1.86), 0.26,0.49,1.23,1.97,2.71$.

${ }^{2} \mathrm{~F}_{4}{ }^{-4} \mathrm{P}^{\prime}{ }_{3}(0.23,0.69,1.14), 0.00,0.46,0.91,1.37,1.83,2.29$.

${ }^{2} \mathrm{~F}_{3}-4 \mathrm{D}_{2}(0.17,0.51), 0.34,0.69,1.03,1.37$.

${ }^{2} \mathrm{~F}_{3}-{ }^{4} \mathrm{D}_{3}(0.26,0.77,1.29), 0.09,0.60,1.12,1.63,2.14$.

${ }^{2} \mathrm{~F}_{3}-4 \mathrm{D}_{4}(\mathbf{0 . 2 9}, 0.86,1.43), 0.00,0.57,1.14,1.71,2.29,2.86$.

${ }^{2} \mathrm{~F}_{4}-4 \mathrm{D}_{3}(0.11,0.34,0.57), 0.57,0.80,1.03,1.26,1.49,1.71$

${ }_{2} \mathrm{~F}_{4}{ }^{-4} \mathrm{D}_{4}(0.14,0.43,0.71,1.00), 0.43,0.71,1.00,1.29,1.57,1.86,2.14$.

${ }^{2} \mathrm{~F}_{3}-4 \mathrm{~F}_{2}{ }_{2}(\mathbf{0 . 2 3}, 0.69), 0.17,0.63,1.09,1.54$.

${ }^{2} \mathrm{~F}_{3}-4 \mathrm{~F}^{\prime}{ }_{3}(0.09,0.26,0.43), 0.60,0.77,0.94,1.12,1.29$.

${ }^{2} \mathrm{~F}_{3}-4 \mathrm{~F}_{4}^{\prime}(0.19,0.57,0.95), 0.29,0.67,1.05,1.43,1.81,2.19$.

${ }^{2} \mathrm{~F}_{4}-\mathrm{F}_{3}^{\prime}{ }_{3}(0.06,0.17,0.29), 0.86,0.97,1.09, \mathrm{i} .20,1.31,1.43$.

${ }^{2} \mathrm{~F}_{4}-4 \mathrm{~F}_{4}^{\prime}(0.05,0.14,0.24,0.33), 0.90,1.00,1.10,1.19,1.29,1.38,1.48$

${ }^{2} \mathrm{~F}_{4}-4 \mathrm{~F}^{\prime}{ }_{5}(0.10,0.29,0.48,0.67), 0.67,0.86,1.05,1.24,1.43,1.62,1.81,2.00$ 。

${ }^{2} \mathrm{~F}_{3}{ }^{-4} \mathrm{G}_{3}(0.14,0.43,0.71), 0.14,0.43,0.71,1.00,1.29$.

${ }^{2} \mathrm{~F}_{3}{ }^{4} \mathrm{G}_{4}(6.06,0.19,0.32), 0.67,0.79,0.92,1.05,1.18, \mathbf{1 . 3 0}$.

${ }^{2} \mathrm{~F}_{4}{ }^{-4} \mathrm{G}_{3}(0.29,0.86,1.43),-0.29,+0.29,0.86,1.43,2.00,2.5 \%$.

${ }^{2} \mathrm{~F}_{4}-4 \mathrm{C}_{4}(0.08,0.24,0.40, \mathbf{0 . 5 6}), 0.59,0.75,0.91,1.06,1.22,1.38,1.54$.

${ }^{2} \mathrm{~F}_{4^{-4}} \mathrm{G}_{5}(0.02,0.04,0.07,0.10), 1.07,1.10,1.13,1.16,1.19,1.22,1.24,1.27$.

${ }^{2} \mathrm{~F}_{3}-4 \mathrm{H}^{\prime}{ }_{4}(0.10,0.29,0.48), 0.19,0.38,0.57,0.76,0.95,1.14$.

${ }^{2} \mathrm{~F}_{4}{ }^{4} \mathrm{H}^{\prime}{ }_{4}(0.24,0.71,1.19, \mathbf{1 . 6 7}),-0.52,-0.05,+0.43,0.90,1.38,1.86,2.33$.

${ }^{2} \mathrm{~F}_{4}{ }^{-4} \mathrm{H}_{5}^{\prime}(0.09,0.26,0.43,0.61), 0.36,0.54,0.71,0.88,1.06,1.23,1.40,1.58$.

${ }^{2} \mathrm{~F}_{4}-4 \mathrm{I}_{5}(0.21,0.62,1.04,1.46),-0.73,-0.31,+0.10,0.52,0.94,1.35,1.77,2.18$.

${ }^{2} \mathrm{G}_{4}-4 \mathrm{D}_{3}^{\prime}(0.24,0.72,1.21),-0.32,+0.16,0.65,1.13,1.61,2.09$.

${ }^{2} \mathrm{G}_{4}-4 \mathrm{D}_{4}^{\prime}(0.27,0.81,1.35,1.89),-0.46,+0.08,0.62,1.16,1,70,2.24,2.78$.

${ }^{2} \mathrm{G}_{5}{ }^{4} \mathrm{D}^{\prime}{ }_{4}(0.16,0.48,0.79,1.11), 0.00,0.32,0.63,0.95,1.27,1.59,1.90,2.22$.

${ }^{2} \mathrm{G}_{4-4} \mathrm{~F}_{3}(0.07,0.21,0.35), 0.54,0.68,0.82,0.96,1.10,1.24$.

${ }^{2} \mathrm{G}_{4}-4 \mathrm{~F}_{4}(0.17,0.52,0.87,1.22), 0.02,0.37,0.71,1.06,1.41,1.76,2.11$.

${ }^{2} \mathrm{G}_{4}-4 \mathrm{~F}_{5}(0.22,0.67,1.11,1.56),-0.22,+0.22,0.67,1.11,1.56,2.00,2.44,2.89$.

${ }^{2} \mathrm{G}_{5}-4 \mathrm{~F}_{4}(0.06,0.19,0.32,0.44), 0.37,0.80,0.92,1.05,1.18,1.30,1.43,1.56$.

${ }^{2} \mathrm{G}_{5}{ }^{4} \mathrm{~F}_{5}(0.11,0.33,0.56,0.78,1.00), 0.33,0.56,0.78,1.00,1.22,1.44,1.67,1.89,2.11$.

${ }^{2} \mathrm{G}_{4}-4 \mathrm{G}^{\prime}{ }_{3}(0.1 \mathrm{C}, 0.48,0.79), 0.10,0.41,0.73,1.05,1.37,1.68$.

${ }^{2} \mathrm{G}_{4^{-4}} \mathrm{G}^{\prime}{ }_{4}(0.05,0.14,0.24,0.33), 0.65,0.75,0.84,0.84,1.03,1.13,1.22$.

${ }^{2} \mathrm{G}_{4}{ }^{-4} \mathrm{G}_{5}{ }_{5}(0.14,0.42,0.71,0.99), 0.18,0.46,0.75,1.03,1.31,1.60,1.88,2.16$.

${ }^{2} \mathrm{G}_{5}-4 \mathrm{G}_{4}{ }_{4}(0.06,0.19,0.32,0.44), 0.67,0.79,0.92,1.05,1.17,1.30,1.43,1.56$.

${ }^{2} \mathrm{G}_{5}{ }^{4} \mathrm{G}^{\prime}{ }_{5}(0.03,0.09,0.15,0.21,0.27), 0.90,0.96,1.02,1.08,1.14,1.20,1.26,1.32,1.38$.

${ }^{2} \mathrm{G}_{5}{ }^{-4} \mathrm{G}_{6}{ }_{6}(0.03,0.24,0.40,0.56,0.73), 0.55,0.71,0.87,1.03,1.19,1.35,1.51,1.68,1.84$, 2.00.

${ }^{2} \mathrm{G}_{4-4} \mathrm{H}_{4}(0.11,0.33,0.56,0.78), 0.11,0.33,0.56,0.78,1.00,1.22,1.44$.

${ }^{2} \mathrm{G}_{4}{ }^{-} \mathrm{H}_{5}(0.04,0.12,0.20,0.28), 0.69,0.77,0.85,0.93,1.01,1.09,1.17,1.25$.

${ }^{2} \mathrm{G}_{5}-{ }^{-4} \mathrm{H}_{4}(0.22,0.67,1.11,1.56),-0.44,0.00,0.44,0.89,1.33,1.78,2.22,2.6 \%$.

${ }^{2} \mathrm{G}_{5}{ }^{-4} \mathrm{H}_{5}(0.07,0.21,0.35,0.49,0.64), 0.47,0.62,0.76,0.90,1.04,1.18,1.32,1.46,1.61$.

${ }^{2} \mathrm{G}_{5}{ }^{-4} \mathrm{H}_{6}(0.01,0.03,0.05,0.08,0.10), 1.04,1.06,1.08,1.10,1.12,1.14,1.17,1.19,1.21,1.23$.

${ }^{2} \mathrm{H}_{5}-4 \mathrm{~F}_{4}^{\prime}(0.16,0.49,0.82,1.15),-0.24,+0.09,0.41,0.74,1.07,1.40,1.73,2.06$.

${ }^{2} \mathrm{H}_{5}-4 \mathrm{~F}_{5}{ }_{5}(0.21,0.64,1.06,1.49,1.91),-0.58,-0.15,+0.27,0.70,1.12,1.54,1.97,2.39,2.82$.

${ }^{2} \mathrm{H}_{6}{ }^{-4} \mathrm{~F}_{5}^{\prime}(0.12,0.36,0.61,0.85,1.09), 0.00,0.24,0.48,0.73,0.97,1.21,1.46,1.70,1.94,2.18$.

${ }^{2} \mathrm{H}_{5}-4 \mathrm{G}_{4}(0.04,0.11,0.19,0.26), 0.65,0.72,0.80,0.87,0.95,1.02,1.10,1.17$.

${ }^{2} \mathrm{H}_{5}{ }^{-4} \mathrm{G}_{5}(0.13,0.39,0.66,0.92,1.18),-0.02,+0.25,0.51,0.78,1.04,1.30,1.56,1.83,2.07$.

${ }^{2} \mathrm{H}_{5}{ }^{-4} \mathrm{G}_{6}(\mathbf{0 . 1 8}, 0.54,0.91,1.27,1.64),-0.36,0.00,0.36,0.73,1.09,1.45,1.82,2.18,2.54,2.91$.

${ }^{2} \mathrm{H}_{\mathrm{B}^{-}-4} \mathrm{G}_{5}(\mathbf{0 . 0 4}, 0.12,0.20,0.28,0.36), \mathbf{0 . 7 3}, 0.81,0.89,0.97,1.05,1.13,1.21,1.29,1.37,1.45$.

${ }^{2} \mathrm{H}_{0^{-4}} \mathrm{G}_{6}(0.09,0.27,0.45,0.64,0.82,1.00), 0.27,0.45,0.64,0.82,1.00,1.18,1.36,1.55,1.73,1.91,2.09$. 
TABLE 5.-Theoretical Zeeman effects (doublet-quartet intersystem)-Continued

${ }^{2} \mathrm{H}_{5}-4 \mathrm{H}_{4}^{\prime}(0.12,0.36,0.60,0.85), 0.06,0.30,0.55,0.79,1.03,1.27,1.51,1.75$.

${ }^{2} \mathrm{H}_{5}-{ }^{-4} \mathrm{H}_{5}{ }_{5}(0.03,0.09,0.15,0.22,0.28), 0.69,0.75,0.82,0.88,0.94,1.00,1.06,1.12,1.19$.

${ }^{2} \mathrm{H}_{5}{ }^{-4} \mathrm{H}_{6}^{\prime}(0.11,0.34,0.56,0.79,1.02), 0.12,0.34,0.57,0.80,1.02,1.25,1.47,1.70,1.91,2.15$.

${ }^{2} \mathrm{H}_{0}{ }^{-4} \mathrm{H}_{5}{ }_{5}(0.06,0.18,0.30,0.43,0.55), 0.54,0.67,0.79,0.91,1.03,1.15,1.27,1.40,1.52,1.64$.

${ }^{2} \mathrm{H}_{\theta^{-4}} \mathrm{H}_{6}^{\prime}(0.02,0.06,0.10,0.15,0.19,0.23), 0.90,0.94,0.99,1.03,1.07,1.11,1.15,1.20,1.24,1.28,1.32$.

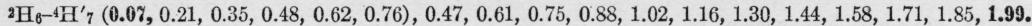

TABLE 6.-Theoretical Zeeman effects (quartet-sextet intersystem)

${ }_{4}^{4} \mathrm{~S}_{2}-\mathrm{BS}_{3}{ }_{3}(0.00), 2.00$.

${ }^{4} \mathrm{~S}_{2}{ }^{-6} \mathrm{P}_{2}(0.20,0.60), 1.80,2.20,2.60$.

${ }^{4} \mathrm{~S}_{2}{ }^{-6} \mathrm{P}_{3}(0.06,0.17), 1.71,1.83,1.94,2.06$.

${ }^{4} \mathrm{~S}_{2}-6 \mathrm{D}^{\prime}{ }_{1}(0.67), 1.33,2.67$.

${ }^{4} \mathrm{~S}_{2}-{ }^{6} \mathrm{D}_{2}^{\prime}{ }_{2}(0.07,0.20), 1.80,1.93,2.07$.

${ }_{4}^{4} \mathrm{~S}_{2}-6 \mathrm{D}^{\prime}{ }_{3}(0.17,0.51), 1.14,1.49,1.83,2.17$.

${ }^{4} \mathrm{~S}_{2}-6 \mathrm{~F}_{1}$ (1.33), 0.67, 3.33 .

${ }^{4} \mathrm{~S}_{2}-6 \mathrm{~F}_{2}(0.47,1.40), 0.60,1.53,2.47$.

${ }^{4} \mathrm{~S}_{2}{ }^{-8} \mathrm{~F}_{3}(0.34,1.03), 0.29,0.97,1.66,2.34$.

${ }^{4} \mathrm{P}_{2}-6 \mathrm{~S}_{3}(0.13,0.40), 1.60,1.87,2.13,2.40$.

${ }^{4} \mathrm{P}_{3}{ }^{-6} \mathrm{~S}_{3}(0.20,0.60,1.00), 1.00,1.40,1.80,2.20,2.60$.

${ }^{4} \mathrm{P}_{1}-6 \mathrm{P}^{\prime}{ }_{2}(0.13), 2.2 \%, 2.53$.

${ }^{4} \mathrm{P}_{2}-6 \mathrm{P}^{\prime}{ }_{2}(0.33,1.00), 1.40,2.07,2.73$.

${ }^{4} \mathrm{P}_{2}-6 \mathrm{P}^{\prime}{ }_{3}$ (0.08, 0.23), 1.16, 1.81, 1.96, 2.11.

${ }^{4} \mathrm{P}_{3}{ }^{-6} \mathrm{P}_{2}^{\prime}(0.40,1.20), 0.40,1.20,2.00,2.80$.

${ }^{4} \mathrm{P}_{3}-6 \mathrm{P}^{\prime}{ }_{3}(0.14,0.43,0.71), 1.17,1.46,1.74,2.02,2.31$.

${ }^{4} \mathrm{P}_{3}-6 \mathrm{P}_{4}^{\prime}(0.06,0.17,0.29), 1.43,1.54,1.66,1.77,1.88$, 2.00.

$\mathrm{P}_{1}{ }^{-6} \mathrm{D}_{1}(0.33), 3.00$.

${ }^{4} \mathrm{P}_{1}{ }^{6} \mathrm{D}_{2}(0.40), 1.47,2.27$.

${ }^{4} \mathrm{P}_{2}-6 \mathrm{D}_{1}(0.80), 0.93,2.53$.

${ }^{4} \mathrm{P}_{2}{ }^{-6} \mathrm{D}_{2}(0.07,0.20), 1.67,1.80,1.93$.

${ }^{4} \mathrm{P}_{2}-6 \mathrm{D}_{3}(0.04,0.11), 1.34,1.62,1.70,1.77$.

${ }^{1} \mathrm{P}_{3}{ }^{-6} \mathrm{D}_{2}(0.13,0.40), 1.20,1.47,1.73,2.00$.

${ }^{4} \mathrm{P}_{3}-6 \mathrm{D}_{3}(0.03,0.09,0.14), 1.51,1.57,1.63,1.68,1.74$.

${ }^{4} \mathrm{P}_{3}-{ }^{6} \mathrm{D}_{4}(0.01,0.02,0.03), 1.56,1.57,1.58,1.59,1.61,1.62$.

${ }^{4} \mathrm{P}_{1}-6 \mathrm{~F}^{\prime}{ }_{1}(1.67), 1.00$.

${ }^{4} \mathrm{P}_{1}{ }^{6} \mathrm{~F}^{\prime}{ }_{2}(0.80), \mathbf{0 . 2 7}, 1.87$.

${ }^{4} \mathrm{P}_{2}-6 \mathrm{~F}^{\prime}{ }_{1}(1.20), 0.53,2.93$.

${ }^{4} \mathrm{P}_{2}-6 \mathrm{~F}_{2}(0.33,1.00), 0.73,1.40,2.07$.

${ }_{4}^{4} \mathrm{P}_{2}-6 \mathrm{~F}^{\prime}{ }_{3}(0.21,0.63), 0.69,1.10,1.52,1.94$.

${ }^{4} \mathrm{P}_{3}-6 \mathrm{~F}^{\prime}{ }_{2}(0.27,0.80), 0.80,1.33,1.87,2.40$.

${ }_{4}^{4} \mathrm{P}_{3}-6 \mathrm{~F}^{\prime}{ }_{3}(0.14,0.43,0.71), 0.89,1.17,1.46,1.74,2.03$.

${ }^{4} \mathrm{P}_{3}{ }^{-6} \mathrm{~F}_{4}^{\prime}(0.10,0.30,0.51), 0.89,1.09,1.30,1.50,1.70,1.90$.

${ }^{4} \mathrm{D}_{2}-{ }^{6} \mathrm{~S}_{3}{ }_{3}(0.40,1.20), 0.80,1.60,2.40,3.20$.

${ }^{4} \mathrm{D}_{3}{ }^{-}{ }^{-} \mathrm{S}^{\prime}{ }_{3}(0.30,0.94,1.57), 0.43,1.06,1.69,2.31,2.94$.

${ }^{4} \mathrm{D}_{4}-6 \mathrm{~S}^{\prime}{ }_{3}(0.29,0.86,1.43), 0.00,0.57,1.14,1.71,2.28,2.86$.

${ }^{4} \mathrm{D}_{1}{ }^{-6} \mathrm{P}_{2}(1.20), 1.20, \mathbf{3 . 6 0}$.

${ }^{4} \mathrm{D}_{2}-6 \mathrm{P}_{2}(0.60,1.80), 0.60,1.80,3.00$.

${ }^{4} \mathrm{D}_{2}-{ }^{-} \mathrm{P}_{3}(6.34,1.03), 0.86,1.54,2.23,2.91$.

${ }^{4} \mathrm{D}_{3}{ }^{-6} \mathrm{P}_{2}(0.51,1.54),-0.17,+0.86,1.88,2.91$.

${ }^{4} \mathrm{D}_{3}-{ }^{-6} \mathrm{P}_{3}(0.26,0.77,1.28), 0.60,1.11,1.63,2.14,2.66$.

${ }^{4} \mathrm{D}_{3}{ }^{-6} \mathrm{P}_{4}(\mathbf{0 . 1 7}, 0.51,0.86), 0.86,1.20,1.54,1.88,2.23,2.5 \%$

${ }^{4} \mathrm{D}_{4}{ }^{-6} \mathrm{P}_{3}(0.23,0.69,1.14), 0.29,0.74,1.20,1.66,2.11,2.57$.

${ }^{4} \mathrm{D}_{4}{ }^{-6} \mathrm{P}_{4}(0.14,0.43,0.71,1.00), 0.71,1.00,1.28,1.5 \%, 1.85,2.14,2.43$.

${ }^{4} \mathrm{D}_{1}{ }^{6} \mathrm{D}^{\prime}{ }_{1}$ (1.67), 1.67 .

${ }^{4} \mathrm{D}_{1}{ }^{6} \mathrm{D}_{2}^{\prime}$ (0.93), 0.93, 2.80.

${ }^{4} \mathrm{D}_{2}-{ }^{6} \mathrm{D}_{1}^{\prime}(1.07), 0.13,2.27$. 
TABLE 6.-Theoretical Zeeman effects (quartet-sextet intersystem)-Continued

${ }^{4} \mathrm{D}_{2}{ }^{-6} \mathrm{D}_{2}^{\prime}(0.33,1.09), 0.87,1.53,2.20$.

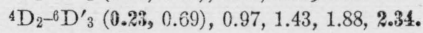

${ }^{4} \mathrm{D}_{3}-6 \mathrm{D}^{\prime}{ }_{2}(0.25,0.74), 0.63,1.12,1.62,2.11$.

${ }^{4} \mathrm{D}_{3}-6 \mathrm{D}^{\prime}{ }_{3}(0.14,0.43, \mathbf{0 . 7 1}), 0.94,1.23,1.51,1.80,2.08$.

${ }^{4} \mathrm{D}_{3}{ }^{-6} \mathrm{D}_{4}^{\prime}(0.11,0.32,0.54), 1.05,1.26,1.48,1.70,1.91,2.13$.

${ }^{4} \mathrm{D}_{4^{-6} \mathrm{D}_{3}^{\prime}}(0.11,0.34,0.57), 0.86,1.08,1.31,1.54,1.77,2.00$.

${ }^{4} \mathrm{D}_{4}{ }^{-6} \mathrm{D}_{4}^{\prime}(0.08,0.24,0.40,0.56), 1.03,1.19,1.35,1.51,1.67,1.83,1.99$.

${ }^{4} \mathrm{D}_{4^{-6}-\mathrm{D}^{\prime}}{ }_{5}(0.06,0.19,0.32,0.44), 1.11,1.24,1.36,1.49,1.62,1.75,1.87,2.00$.

${ }^{4} \mathrm{D}_{1-0}{ }^{0} \mathrm{~F}_{1}(0.33), 0.33$.

${ }^{4} \mathrm{D}_{1}{ }^{-6} \mathrm{~F}_{2}(0.53), 0.53, \mathbf{1 . 6 0}$.

${ }^{4} \mathrm{D}_{2}{ }^{-6} \mathrm{~F}_{1}(0.93), 0.27,2.13$.

${ }^{4} \mathrm{D}_{2}{ }^{-6} \mathrm{~F}_{2}(0.07,0.20), 1.00,1.13,1.27$.

${ }^{4} \mathrm{D}_{2}{ }^{-6} \mathrm{~F}_{3}(0.06,0.17), 1.14,1.26,1.37,1.49$.

${ }^{4} \mathrm{D}_{3}{ }^{-6} \mathrm{~F}_{2}(0.15,0.46), 0.91,1.22,1.52,1.83$.

${ }^{4} \mathrm{D}_{3}{ }^{-6} \mathrm{~F}_{3}(0.03,0.09,0.14), 1.23,1.29,1.34,1.40,1.46$.

${ }^{4} \mathrm{D}_{3}{ }^{-6} \mathrm{~F}_{4}(0.01,0.04,0.06), 1.33,1.36,1.38,1.41,1.43,1.46$.

${ }^{4} \mathrm{D}_{4}^{-{ }^{-1}} \mathrm{~F}_{3}(0.06,0.17,0.29), 1.14,1.26,1.37,1.48,1.60,1.71$.

${ }^{4} \mathrm{D}_{4}{ }^{-6} \mathrm{~F}_{4}(0.02,0.05,0.08,0.11), 1.32,1.35,1.38,1.41,1.44,1.48,1.51$.

${ }^{4} \mathrm{D}_{4}{ }^{-6} \mathrm{~F}_{5}(0.00,0.01,0.01,0.02), 1.41,1.42,1.42,1.43,1.44,1.44,1.45,1.46$.

${ }^{4} \mathrm{D}_{1^{-6}} \mathrm{G}_{2}^{\prime}(0.00), 0.00$.

${ }^{4} \mathrm{D}_{2}-{ }^{6} \mathrm{G}_{2}^{\prime}(0.60, \mathbf{1 . 5 0}),-0.60,+\mathbf{0 . 6 0}, 1.80$.

${ }^{4} \mathrm{D}_{2}{ }^{-6} \mathrm{G}^{\prime}{ }_{3}(0.17,0.51), 0.34,0.69,1.03,1.37$.

${ }^{4} \mathrm{D}_{3}{ }^{-6} \mathrm{G}^{\prime}{ }_{2}(\mathbf{0 . 6 9}, 2.06),-0.69,+0.69,2.06,3.43$.

${ }^{4} \mathrm{D}_{3}{ }^{6} \mathrm{G}^{\prime}{ }_{3}(0.26,0.77,1.28), 0.09,0.60,1.11,1.63,2.14$.

${ }^{4} \mathrm{D}_{3}{ }^{-6} \mathrm{G}^{\prime}{ }_{4}(\mathbf{0 . 1 1}, 0.34,0.57), \mathbf{0 . 5} \%, 0.80,1.03,1.26,1.48,1.71$.

${ }^{4} \mathrm{D}_{4}{ }^{-6} \mathrm{G}_{3}{ }_{3}(0.29,0.86,1.43), 0.00,0.57,1.14,1.71,2.29,2.86$.

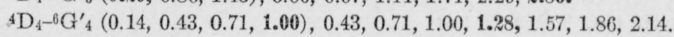

${ }^{4} \mathrm{D}_{4}{ }^{-6} \mathrm{G}^{\prime}{ }_{5}(0.08,0.23,0.39,0.54), 0.73,0.88,1.04,1.19,1.35,1.50,1.66,1.82$.

${ }^{4} \mathrm{~F}_{2}-\mathrm{CS}_{3}(0.80,2.40),-0.40,+1.20,2.80,4.40$,

${ }^{4} \mathrm{~F}_{3}{ }^{-6} \mathrm{~S}_{3}(0.48,1.46,2.43),-0.43,+0.54,1.51,2.48,3.46$.

${ }^{4} \mathrm{~F}_{4}{ }^{-6} \mathrm{~S}_{3}(0.38,1.14,1.90),-0.67,+0.10,0.86,1.62,2.38,3.14$.

${ }_{4}^{4} \mathrm{~F}_{2}-{ }^{-} \mathrm{P}_{2}^{\prime}(1.00,3.00),-0.60,+\mathbf{1 . 4 0} 3.40$.

${ }^{4} \mathrm{~F}_{2}-6 \mathrm{P}_{3}{ }_{3}(\mathbf{0 . 7 4}, 2.23),-0.34,+1.14,2.63,4.11$.

${ }^{4} \mathrm{~F}_{3}{ }^{6} \mathrm{P}^{\prime}{ }_{2}(0.69,2.06),-\mathbf{1 . 0 3},+0.34,1.71,3.09$.

${ }^{4} \mathrm{~F}_{3}{ }^{-6} \mathrm{P}_{3}^{\prime}(0.43,1.29,2.14),-0.26,+0.60,1.46,2.32,3.17$.

${ }_{4}^{4} \mathrm{~F}_{3}-6 \mathrm{P}_{4}^{\prime}(0.34,1.03,1.71), 0.00,0.69,1.37,2.06,2.74,3.43$.

${ }^{4} \mathrm{~F}_{1}{ }^{-6} \mathrm{P}^{\prime}{ }_{3}(0.32,0.97,1.62),-0.38,+0.27,0.91,1.56,2.21,2.86$.

${ }^{4} \mathrm{~F}_{4}-6 \mathrm{P}_{4}^{\prime}(0.24,0.71,1.19,1.67), 0.05,0.52,1.00,1.47,1.95,2.43,2.90$.

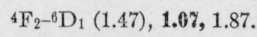

${ }_{4}^{4} \mathrm{~F}_{2}-6 \mathrm{D}_{2}(0.73,2.2 \theta),-0.33,+1.13 .2 .60$.

${ }^{4} \mathrm{~F}_{2}{ }^{-6} \mathrm{D}_{3}(\boldsymbol{\theta} .63 .1 .88),-0.23,+1.03,2.28,3.54$.

${ }^{4} \mathrm{~F}_{3}-6 \mathrm{D}_{2}(0.42,1.26),-0.23,+0.61,1.45,2.29$.

${ }^{4} \mathrm{~F}_{3}{ }^{-6} \mathrm{D}_{3}(0.31,0.94,1.5 \%), 0.09,0.71,1.34,1.97,2.60$.

${ }^{4} \mathrm{~F}_{3}{ }^{-6} \mathrm{D}_{4}(0.28,0.84,1.40), 0.19,0.75,1.31,1.87,2.42,2.98$.

${ }^{4} \mathrm{~F}_{4}{ }^{-6} \mathrm{D}_{3}(0.21,0.63,1.05), \mathbf{0 . 1 9}, 0.61,1.03,1.45,1.87,2.28$.

${ }^{4} \mathrm{~F}_{4}{ }^{-6} \mathrm{D}_{4}(0.17,0.52,0.87,1.22), 0.36,0.71,1.06,1.41,1.76,2.11,2.46$.

${ }^{4} \mathrm{~F}_{4}{ }^{-6} \mathrm{D}_{5}(\mathbf{0 . 1 6}, 0.48,0.79,1.11), 0.44,0.76,1.08,1.40,1.72,2.03,2.35,2.67$.

${ }^{4} \mathrm{~F}_{5}{ }^{-6} \mathrm{D}_{4}(\mathbf{0 . 1 3}, 0.38,0.64,0.89), \mathbf{0 . 4 4}, 0.70,0.95,1.21,1.46,1.71,1.97,2.22$.

${ }^{4} \mathrm{~F}_{5}{ }^{-6} \mathrm{D}_{5}(0.11,0.33,0.56,0.78,1.00), 0.56,0.78,1.00,1.22,1.44 .1 .67,1.89,2.11,2.33$.

${ }^{4} \mathrm{~F}_{2}-6 \mathrm{~F}^{\prime}{ }_{1}(0.53),-0.13,+0.93$.

${ }^{4} \mathrm{~F}_{2}-6 \mathrm{~F}_{2}{ }_{2}(0.33,1.00), 0.07,0.73,1.40$.

${ }^{4} \mathrm{~F}_{2}-6 \mathrm{~F}^{\prime}{ }_{3}(0.46,1.37),-0.06,+0.86,1.77,2.68$.

${ }^{4} \mathrm{~F}_{3}-6 \mathrm{~F}_{2}{ }_{2}(\mathbf{0 . 0 2}, 0.06), \mathbf{0 . 9 7}, 1.01,1.05,1.09$.

${ }^{4} \mathrm{~F}_{3}-6 \mathrm{~F}^{\prime}{ }_{3}(0.14,0.43,0.71), 0.60,0.89,1.17,1.46,1.74$.

${ }^{4} \mathrm{~F}_{3}{ }^{-6} \mathrm{~F}^{\prime}{ }_{4}(0.18,0.55,0.92), 0.48,0.84,1.21,1.58,1.95$, 2.32.

${ }^{4} \mathrm{~F}_{4}{ }^{-6} \mathrm{~F}^{\prime}{ }_{3}(0.04,0.11,0.19), 1.05,1.12,1.20,1.28,1.35,1.43$.

${ }^{4} \mathrm{~F}_{4}-{ }^{-6} \mathrm{~F}^{\prime}{ }_{4}(0.08,0.24,0.40, \mathbf{0 . 5 6}), 0.84,1.00,1.16, \mathbf{1 . 3 2}, 1.48,1.64,1.79$.

${ }^{4} \mathrm{~F}_{4}{ }^{-6} \mathrm{~F}^{\prime}{ }_{5}(\mathbf{0 . 1 0}, 0.29,0.49,0.69), 0.75,0.94,1.14,1.34,1.53,1.73,1.93,2.12$.

${ }^{4} \mathrm{~F}_{5}-6 \mathrm{~F}^{\prime}{ }_{4}(\mathbf{0 . 0 3}, 0.10,0.16,0.22), \mathbf{1 . 1 1}, 1.17,1.24,1.30,1.36,1.43,1.49,1.56$.

${ }^{4} \mathrm{~F}_{5}-{ }^{-6} \mathrm{~F}^{\prime}{ }_{5}(0.05,0.15,0.25,0.35,0.45), 0.98,1.08,1.18,1.28,1.38,1.48,1.59,1.69,1.79$.

${ }^{4} \mathrm{~F}_{5}{ }^{-6} \mathrm{~F}^{\prime}{ }_{6}(0.06,0.18,0.30,0.42,0.55), 0.91,1.03,1.15,1.27,1.40,1.52,1.64,1.76,1.88,2.00$. 
TABLE 6.-Theoretical Zeeman effects (quartet-sextet intersystem)-Continued

${ }^{4} \mathrm{~F}_{2}-6 \mathrm{G}_{2}(0.20,0.60),-0.20,+0.20,0.60$.

${ }^{4} \mathrm{~F}_{2}{ }^{-6} \mathrm{G}_{3}(0.23,0.69), 0.17,0.63,1.08,1.54$.

${ }^{4} \mathrm{~F}_{3}-{ }^{6} \mathrm{G}_{2}(0.51,1.54),-0.51,+0.51,1.54,2.5 \%$.

${ }^{4} \mathrm{~F}_{3}-{ }^{6} \mathrm{G}_{3}(0.09,0.26,0.43), 0.60,0.77,0.94,1.11,1.28$.

${ }^{4} \mathrm{~F}_{3}-{ }^{6} \mathrm{G}_{4}(\mathbf{0 . 0 6}, 0.17,0.29), 0.86,0.97,1.20,1.31,1.43$.

${ }^{4} \mathrm{~F}_{4}{ }^{-6} \mathrm{G}_{3}(0.19,0.57,0.95), 0.29,0.67,1.05,1.43,1.81, \mathbf{2 . 1 9}$.

${ }^{4} \mathrm{~F}_{4}{ }^{-6} \mathrm{G}_{4}(0.05,0.14,0.24,0.33), 0.90,1.00,1.09,1.19,1.28,1.38,1.48$.

${ }^{4} \mathrm{~F}_{4^{-6}} \mathrm{G}_{5}(0.02,0.05,0.09,0.12), 1.15,1.18,1.22,1.25,1.29,1.32,1.36,1.39$.

${ }^{4} \mathrm{~F}_{5}-{ }^{6} \mathrm{G}_{4}(0.10,0.29,0.48,0.67), 0.67,0.86,1.05,1.24,1.43,1.62,1.81,2.00$.

${ }^{4} \mathrm{~F}_{5}{ }^{-6} \mathrm{G}_{5}(0.03,0.09,0.15,0.21,0.27), 1.06,1.12,1.18,1.24,1.30,1.36,1.42,1.48,1.55$.

${ }^{4} \mathrm{~F}_{5}{ }^{-6} \mathrm{G}_{6}(0.00,0.01,0.02,0.03,0.04), 1.30,1.31,1.32,1.33,1.34,1.35,1.36,1.37,1.38,1.39$.

${ }^{4} \mathrm{G}_{3}{ }^{-6} \mathrm{~F}_{2}(\mathbf{0 . 2 5}, 0.74),-0.17,+0.32,0.82,1.31$.

${ }^{4} \mathrm{G}_{3}-6 \mathrm{~F}_{3}(0.37,1.11,1.86),-0.54,+0.20,0.94,1.68,2.43$.

${ }^{4} \mathrm{G}_{3}-6 \mathrm{~F}_{4}(0.41,1.24,2.06),-0.66,+0.16,0.98,1.81,2.63$, 3.46.

${ }^{4} \mathrm{G}_{4}-6 \mathrm{~F}_{3}(\mathbf{0 . 1 6}, 0.49,0.82), \mathbf{0 . 1 6}, 0.49,0.82,1.15,1.48,1.81$.

${ }^{4} \mathrm{G}_{4^{-6} \mathrm{~F}_{4}}(0.21,0.62,1.03,1.44),-0.05,+0.36,0.78,1.19,1.60,2.01,2.43$.

${ }^{4} \mathrm{G}_{4}-6 \mathrm{~F}_{5}(0.22,0.67,1.12,1.57),-0.14,+0.31,0.76,1.21,1.66,2.11,2.56,3.01$.

${ }^{4} \mathrm{G}_{5}-6 \mathrm{~F}_{4}(0.11,0.34,0.56,0.79), 0.39,0.61,0.84,1.06,1.28,1.51,1.73,1.96$.

${ }^{4} \mathrm{G}_{5}-6 \mathrm{~F}_{5}(0.13,0.39,0.66,0.92,1.18), 0.25,0.52,0.78,1.04,1.30,1.56,1.83,2.09,2.35$.

${ }^{4} \mathrm{G}_{5}-6 \mathrm{~F}_{6}(0.14,0.42,0.71,0.99,1.28), 0.18,0.46,0.75,1.03,1.31,1.60,1.88,2.17,2.45,2.73$.

${ }^{4} \mathrm{G}_{6}{ }^{-6} \mathrm{~F}_{5}(\mathbf{0 . 0 8}, 0.24,0.40,0.57,0.73), 0.54,0.71,0.87,1.03,1.19,1.35,1.51,1.68,1.84,2.00$.

${ }^{4} \mathrm{G}_{6}{ }^{-6} \mathrm{~F}_{6}(0.09,0.27,0.46,0.64,0.83,1.01), 0.44,0.63,0.81,1.00,1.18,1.36,1.55,1.73,1.92,2.10,2.28$.

${ }^{4} \mathrm{G}_{3}-6 \mathrm{G}_{2}{ }_{2}(\mathbf{0 . 2 9}, 0.86),-0.29,+0.29,0.86,1.43$.

${ }^{4} \mathrm{G}_{3}-6 \mathrm{CG}_{3}{ }_{3}(0.14,0.43,0.71), 0.15,0.43,0.71,1.00,1.28$,

${ }^{4} \mathrm{G}_{3}-6 \mathrm{G}^{\prime}{ }_{4}(\boldsymbol{\theta} .29,0.86,1.43),-0.29,+0.29,0.86,1.43,2.00$, 2.5\%.

${ }^{4} \mathrm{G}_{4}{ }^{6} \mathrm{G}^{\prime}{ }_{3}(\mathbf{0 . 0 6}, 0.19,0.32), 0.66,0.79,0.92,1.05,1.17,1.30$.

${ }^{4} \mathrm{G}_{4}-6 \mathrm{G}^{\prime}{ }_{4}(0.08,0.24,0.40,0.56), 0.58,0.74,0.90,1.06,1.22,1.38,1.54$.

${ }^{4} \mathrm{G}_{4}{ }^{-6} \mathrm{G}^{\prime}{ }_{5}(\mathbf{0 . 1 4}, 0.43,0.72,1.01), 0.26,0.55,0.84,1.13,1.42,1.70,1.99$, 2.28.

${ }^{4} \mathrm{G}_{5}-6 \mathrm{G}_{4}^{\prime}(\mathbf{0 . 0 1}, 0.04,0.07,0.10), 1.07,1.10,1.13,1.16,1.19,1.21,1.24,1.27$.

${ }^{4} \mathrm{G}_{5}-6 \mathrm{G}^{\prime}{ }_{5}(0.05,0.15,0.25,0.35,0.45), 0.82,0.92,1.02,1.12,1.22,1.32,1.42,1.52,1.62$

${ }^{4} \mathrm{G}_{5}{ }^{6} \mathrm{G}^{\prime}{ }_{6}(0.08,0.26,0.43,0.60,0.77), 0.57,0.74,0.91,1.08,1.26,1.43,1.60,1.77,1.95,2.12$.

${ }^{4} \mathrm{G}_{6}{ }^{6} \mathrm{G}^{\prime}{ }_{5}(0.00), 1.27$.

${ }^{4} \mathrm{G}_{6^{-}-6 \mathrm{G}^{\prime}}{ }_{6}(0.04,0.11,0.18,0.25,0.32,0.40), 0.95,1.02,1.09,1.16,1.24,1.31,1.38,1.45,1.52,1.60,1.67$.

${ }^{4} \mathrm{C}_{\mathrm{C}_{6}-8}{ }^{8} \mathrm{G}^{\prime}{ }_{7}(0.06,0.17,0.28,0.39,0.50,0.61), 0.77,0.88,0.99,1.10,1.22,1.33,1.44,1.55,1.66,1.78,1.89$, 2.00.

${ }^{4} \mathrm{G}_{2}{ }^{6} \mathrm{H}_{3}(0.14,0.43,0.71),-0.14,+0.14,0.43,0.71,1.00$.

${ }^{4} \mathrm{G}_{3}-{ }^{6} \mathrm{H}_{4}(0.13,0.38,0.63), 0.19,0.45,0.70,0.95,1.20,1.15$.

${ }^{4} \mathrm{G}_{4}-{ }^{6} \mathrm{H}_{3}(0.35,1.05,1.74),-0.76,-0.06,+0.63,1.33,2.03,2.73$.

${ }^{4} \mathrm{G}_{4}-6 \mathrm{H}_{4}(0.08,0.24,0.40,0.56), 0.42,0.58,0.74,0.90,1.06,1.22,1.38$

${ }^{4} \mathrm{G}_{4}-6 \mathrm{H}_{5}(0.04,0.13,0.22,0.31), 0.76,0.85,0.94,1.03,1.12,1.20,1.29,1.39$.

${ }^{4} \mathrm{G}_{5}{ }^{-6} \mathrm{H}_{4}(\mathbf{0 . 1 7}, 0.52,0.87,1.22),-0.05,+0.30,0.65,1.00,1.35,1.69,2.04,2.39$.

${ }^{4} \mathrm{G}_{5}{ }^{-6} \mathrm{H}_{5}(0.05,0.15,0.25,0.35,0.45), 0.72,0.82,0.92,1.02,1.12,1.22,1.32,1.42,1.52$.

${ }^{4} \mathrm{G}_{5}{ }^{-6} \mathrm{H}_{6}(0.02,0.05,0.08,0.11,0.14), 1.06,1.09,1.12,1.16,1.19,1.22,1.25,1.28,1.32,1.35$.

${ }^{4} \mathrm{G}_{6}{ }^{6}{ }^{6} \mathrm{H}_{5}(0.10,0.30,0.50,0.70,0.90), 0.37,0.57,0.77,0.97,1.17,1.37,1.57,1.77,1.97,2.1 \%$.

${ }^{4} \mathrm{G}_{6}{ }^{-6} \mathrm{H}_{6}(0.03,0.10,0.17,0.21,0.30,0.37), 0.90,0.97,1.03,1.10,1.17,1.24,1.31,1.37,1.44,1.51,1.58$.

${ }^{4} \mathrm{G}_{6}{ }^{-6} \mathrm{H}_{7}(0.005,0.01,0.02,0.03,0.04,0.05), 1.23,1.24,1.25,1.26,1.27,1.28,1.29,1.30,1.31,1.32,1.33,1.34$.

TABLE 7.-Theoretical Zeeman effects (sextet-octet intersystem)

${ }^{6} \mathrm{~S}_{3}-8 \mathrm{~S}_{4}^{\prime}(0.00), 2.00$.

${ }^{6} \mathrm{~S}_{3}-8 \mathrm{P}_{3}(0.14,0.43,0.71), 1.57,1.86,2.14,2.43,2.72$.

${ }^{6} \mathrm{~S}_{3}-8 \mathrm{P}_{4}(0.03,0.10,0.16), 1.78,1.84,1.90,1.97,2.03,2.10$.

${ }^{6} \mathrm{~S}_{3}-8 \mathrm{D}_{2}^{\prime}(0.40,1.20), 0.80,1.60,2.40,3.20$.

${ }^{6} \mathrm{~S}_{3}-8 \mathrm{D}^{\prime}{ }_{3}(0.03,0.09,0.14), 1.91,1.97,2.03,2.09,2.14$.

${ }^{8} \mathrm{~S}_{3}{ }^{-8} \mathrm{D}_{4}^{\prime}(0.10,0.29,0.48), 1.33,1.52,1.71,1.90,2.09,2.28$.

${ }^{6} \mathrm{~S}_{3}-8 \mathrm{~F}_{2}(0.00), 2.00$.

${ }^{6} \mathrm{~S}_{3}-8 \mathrm{~F}_{3}(0.14,0.43,0.71), 1.28,1.57,1.86,2.14,2.43$

${ }^{6} \mathrm{~S}_{3}-8 \mathrm{~F}_{4}(0.19,0.57,0.95), 0.67,1.05,1.43,1.81,2.19,2.57$. 
TABLE 7.-Theoretical Zeeman effects (sextet-octet intersystem)-Continued

${ }^{6} \mathrm{P}_{3}-{ }^{8} \mathrm{~S}_{4}(0.06,0.17,0.29), 1.71,1.83,1.94,2.06,2.17$, 2.29.

${ }^{6} \mathrm{P}_{4}{ }^{-8} \mathrm{~S}_{4}(0.14,0.43,0.71,1.00), 1.00,1.29,1.57,1.86,2.14,2.43,2.72$.

${ }^{6} \mathrm{P}_{2}-8 \mathrm{P}_{3}^{\prime}(0.06,0.17), 2.12,2.23,2.34,2.46$.

${ }^{6} \mathrm{P}_{3}-8 \mathrm{P}_{3}^{\prime}(0.20,0.60,1.91), 1.29,1.69,2.08,2.48,2.88$.

${ }^{6} \mathrm{P}_{3}-8 \mathrm{P}_{4}^{\prime}(0.03,0.08,0.13), 1.81,1.86,1.91,1.96,2.01,2.06$.

${ }^{6} \mathrm{P}_{4}-8 \mathrm{P}^{\prime}{ }_{3}(0.29,0.86,1.43), 0.29,0.86,1.43,2.00,2.57,3.14$.

${ }^{6} \mathrm{P}_{4}-8 \mathrm{P}_{4}^{\prime}(0.11,0.33,0.56,0.78), 1.16,1.38,1.60,1.85 \%$ 2.05, 2.27, 2.49 .

${ }^{6} \mathrm{P}_{4}{ }^{-8} \mathrm{P}_{5}^{\prime}(0.03,0.10,0.16,0.22), 1.56,1.62,1.68,1.75,1.21,1.87,1.94,2.00$ 。

${ }^{6} \mathrm{P}_{2}-{ }^{-8} \mathrm{D}_{2}(0.20,0.60), 2.20,2.60,3.00$.

${ }^{6} \mathrm{P}_{2}-{ }^{8} \mathrm{D}_{3}(9.17,0.51), 1.54,1.88,2.23,2.57$.

${ }^{6} \mathrm{P}_{3}-{ }^{-5} \mathrm{D}_{2}(0.46,1.37), 0.51,1.43,2.34,3.26$.

${ }^{6} \mathrm{P}_{3}-{ }^{8} \mathrm{D}_{3}\left(0.09,0.26,0 .{ }^{43}\right), 1.63,1.80,1.97,2.14,2.32$.

${ }^{6} \mathrm{P}_{3}{ }^{-}{ }^{9} \mathrm{D}_{4}(0.04,0.11,0.19), 1.62,1.70,1.77,1.85,1.92,2.00$.

${ }^{6} \mathrm{P}_{4}-8 \mathrm{D}_{3}(0.17,0.51,0.86), 0.86,1.20,1.54,1.88,2.22,2.57$.

${ }^{6} \mathrm{P}_{4}{ }^{-8} \mathrm{D}_{4}(0.05,0.14,0.24,0.33), 1.48,1.57,1.67,1.76,1.86,1.95,2.05$.

${ }^{6} \mathrm{P}_{4}{ }^{-8} \mathrm{D}_{5}(0.01,0.03,0.04,0.06), 1.61,1.65,1.67,1.69,1.71,1.72,1.74,1.76$.

${ }^{6} \mathrm{P}_{2}-8 \mathrm{~F}^{\prime}{ }_{1}(0.80), \mathbf{1 . 6 0}, 3.20$.

${ }^{6} \mathrm{P}_{2}-8 \mathrm{~F}^{\prime}{ }_{2}(0.20,0.60), 1.80,2.20,2.60$.

${ }^{6} \mathrm{P}_{2}-8 \mathrm{~F}^{\prime}{ }_{3}(0.34,1.03), 0.63,1.37,2.06,2.74$.

${ }^{6} \mathrm{P}_{3}-8 \mathrm{~F}^{\prime}{ }_{2}(0.06,0.17), \mathbf{1 . 7 1}, 1.83,1.94,2.06$.

${ }^{6} \mathrm{P}_{3}-8 \mathrm{~F}^{\prime}{ }_{3}(0.09,0.26,0.43), 1.46,1.63,1.80,1.97,2.14$.

${ }^{0} \mathrm{P}_{3}-8 \mathrm{~F}_{4}^{\prime}(0.13,0.40,0.67), 0.95,1.22,1.49,1.75,2.02,2.28$.

${ }^{6} \mathrm{P}_{4}-8 \mathrm{~F}^{\prime}{ }_{3}(0.00), 1.71$.

${ }^{6} \mathrm{P}_{4^{-8}} \mathrm{~F}_{4}^{\prime}(0.05,0.14,0.24,0.33), 1.38,1.48,1.57,1.67,1.76,1.86,1.95$.

${ }^{6} \mathrm{P}_{4}{ }^{-8} \mathrm{~F}^{\prime}{ }_{5}(0.07,0.21,0.35,0.48), \mathbf{1 . 0 9}, 1.23,1.37,1.51,1.65,1.78,1.92,2.06$.

${ }^{6} \mathrm{D}_{3}-8 \mathrm{~S}_{4}^{\prime}(0.17,0.51,0.86), 1.14,1.48,1.83,2.17,2.52,2.86$.

${ }^{6} \mathrm{D}_{4}-8 \mathrm{~S}_{4}{ }_{4}(0.21,0.62,1.03,1.45), 0.56,0.97,1.38,1.79,2.21,2.62,3.03$.

${ }^{6} \mathrm{D}_{5}-{ }^{8} \mathrm{~S}_{4}^{\prime}(0.22,0.67,1.11,1.56), 0.00,0.44,0.89,1.33,1.78,2.22,2.67,3.11$.

${ }^{6} \mathrm{D}_{2}-{ }^{8} \mathrm{P}_{3}(0.21,0.63), 1.66,2.08,2.50,2.92$.

${ }^{6} \mathrm{D}_{3}{ }^{-} \mathrm{P}_{3}(0.31,0.94,1.57), 0.71,1.34,1.97,2.60,3.23$.

${ }^{6} \mathrm{D}_{3}-8 \mathrm{P}_{4}(\mathbf{0 . 1 4}, 0.42,0.70), 1.24,1.52,1.80,2.08,2.35, \mathbf{2 . 6 3}$.

${ }^{6} \mathrm{D}_{4}-8 \mathrm{P}_{3}(\mathbf{0 . 3 5}, 1.05,1.75),-0.16,+0.54,1.24,1.94,2.64,3.33$.

${ }^{6} \mathrm{D}_{4}{ }^{8} \mathrm{P}_{4}(0.17,0.52,0.87,1.22), 0.71,1.06,1.41,1.76,2.11,2.46,2.81$.

${ }^{6} \mathrm{D}_{4}-{ }^{8} \mathrm{P}_{5}(0.10,0.29,0.48,0.67), 1.11,1.30,1.49,1.68,1.87,2.06,2.25, \mathbf{2 . 4 5}$.

${ }^{6} \mathrm{D}_{5}-{ }^{8} \mathrm{P}_{4}(0.19,0.57,0.95,1.33), 0.22,0.60,0.98,1.36,1.74,2.13,2.51,2.89$.

${ }^{6} \mathrm{D}_{5}{ }^{8} \mathrm{P}_{5}(0.11,0.33,0.55,0.78,1.00), 0.78,1.00,1.22,1.44,1.67,1.89,2.11,2.33,2.55$.

${ }^{6} \mathrm{D}_{1-8}{ }^{8} \mathrm{D}_{2}^{\prime}(0.27), 2.53,3.07$.

${ }^{6} \mathrm{D}_{2}-{ }^{8} \mathrm{D}_{2}{ }_{2}(0.47,1.40), 1.40,2.33,3.27$.

${ }^{0} \mathrm{D}_{2}-8 \mathrm{D}^{\prime}{ }_{3}(0.10,0.29), 1.77,1.96,2.15,2.34$.

${ }^{6} \mathrm{D}_{3}{ }^{8} \mathrm{D}_{2}^{\prime}{ }_{2}(\mathbf{0 . 5} \%, 1.71),-0.06,+1.09,2.23,3.37$.

${ }^{6} \mathrm{D}_{3}-{ }^{8} \mathrm{D}_{3}^{\prime}(0.20,0.60,1.00), 1.06,1.46,1.86,2.26,2.66$.

${ }^{6} \mathrm{D}_{3}-8 \mathrm{D}^{\prime}{ }_{4}(\mathbf{0 . 0 8}, 0.23,0.38), 1.43,1.58,1.73,1.89,2.04, \mathbf{2 . 1 9}$.

${ }^{6} \mathrm{D}_{4}-8 \mathrm{D}_{3}^{\prime}{ }_{3}(0.24,0.70,1.17), 0.41,0.88,1.35,1.82,2.29,2.76$.

${ }^{6} \mathrm{D}_{4}{ }^{-8} \mathrm{D}_{4}^{\prime}(0.11,0.33,0.56,0.78), 1.03,1.25,1.48,1.70,1.92,2.14,2.37$.

${ }^{6} \mathrm{D}_{4}-8 \mathrm{D}_{5}^{\prime}(0.05,0.16,0.27,0.38), 1.31,1.42,1.53,1.64,1.75,1.86,1.97,2.08$.

${ }^{6} \mathrm{D}_{5}-{ }^{-8} \mathrm{D}_{4}^{\prime}(\mathbf{0 . 1 3}, 0.38,0.63,0.89), \mathbf{0 . 6 7}, 0.92,1.17,1.43,1.68,1.94,2.19,2.44$.

${ }^{6} \mathrm{D}_{5}-{ }^{8} \mathrm{D}^{\prime}{ }_{5}(0.07,0.21,0.35,0.49,0.64), 1.06,1.20,1.34,1.48, \mathbf{1 . 6 3}, 1.77,1.91,2.05,2.19$.

${ }^{6} \mathrm{D}_{5}-{ }^{8} \mathrm{D}_{6}^{\prime}(0.04,0.12,0.20,0.28,0.36), 1.27,1.35,1.43,1.51,1.60,1.68,1.76,1.84,1.92,2.00$.

${ }^{6} \mathrm{D}_{1}-8 \mathrm{~F}_{1}(0.33), 3,67$.

${ }^{6} \mathrm{D}_{1}-8 \mathrm{~F}_{2}(0.67), 1.33,2.67$.

${ }^{6} \mathrm{D}_{2}-{ }^{8} \mathrm{~F}_{1}(1.07), 0.80,2.93$.

${ }^{6} \mathrm{D}_{2}-8 \mathrm{~F}_{2}(0.07,0.20), 1.80,1.93,2.07$.

${ }^{6} \mathrm{D}_{2}-8 \mathrm{~F}_{3}(0.08,0.23), 1.49,1.64,1.79,1.94$.

${ }^{6} \mathrm{D}_{3}-8 \mathrm{~F}_{2}(0.17,0.51), 1.14,1.48,1.83,2.17$.

${ }^{0} \mathrm{D}_{3}-8 \mathrm{~F}_{3}(0.03,0.09,0.14), 1.57,1.63,1.69,1.74,1.80$.

${ }^{6} \mathrm{D}_{3}-{ }^{8} \mathrm{~F}_{4}(0.02,0.06,0.10), 1.52,1.56,1.60,1.64,1.68,1.72$.

${ }^{6} \mathrm{D}_{4}-{ }^{-8} \mathrm{~F}_{3}(0.06,0.19,0.32), \mathbf{1 . 2 7}, 1.40,1.52,1.65,1.78,1.90$.

${ }^{6} \mathrm{D}_{4}{ }^{8} \mathrm{~F}_{4}(0.02,0.05,0.08,0.11), 1.51,1.54,1.57,1.60,1.63,1.67,1.70$.

${ }^{6} \mathrm{D}_{4}-8 \mathrm{~F}_{5}(0.01,0.02,0.03,0.04), 1.54,1.55,1.56,1.57,1.58,1.59,1.61,1.62$.

${ }^{6} \mathrm{D}_{5}-8 \mathrm{~F}_{4}(0.03,0.10,0.16,0.22), 1.33,1.40,1.46,1.52,1.59,1.65,1.71,1.78$.

${ }^{6} \mathrm{D}_{5}{ }^{8} \mathrm{~F}_{5}(0.01,0.03,0.05,0.07,0.09), 1.48,1.51,1.53,1.55, \mathbf{1 . 5} \%, 1.59,1.61,1.63,1.65$.

${ }^{6} \mathrm{D}_{5}-8 \mathrm{~F}_{6}(0.00,0.00,0.01,0.01,0.01), 1.54,1.54,1.54,1.54,1.55,1.55,1.56,1.56,1.56,1.57$. 
TABLe 7.-Theoretical Zeeman effects (sextet-octet intersystem)-Continued

${ }^{6} \mathrm{D}_{1}{ }^{-8} \mathrm{G}_{1}^{\prime}(2,33), 1.00$.

${ }^{6} \mathrm{D}_{1}-8 \mathrm{G}^{\prime}{ }_{2}(1.20),-0.27,+2.13$.

${ }^{6} \mathrm{D}_{2}-{ }^{-8} \mathrm{G}^{\prime}{ }_{1}(1.60), 0.27,3.4 \%$.

${ }^{6} \mathrm{D}_{2}-{ }^{8} \mathrm{G}_{2}{ }_{2}(0.47,1.40), 0.47,1.40,2.33$.

${ }^{6} \mathrm{D}_{2}-{ }^{8} \mathrm{G}_{3}{ }_{3}(0.30,0.91), 0.34,0.95,1.56,2.17$.

${ }^{6} \mathrm{D}_{3}-8 \mathrm{G}_{2}{ }_{2}(0.36,1.08), 0.57,1.29,2.02,2.74$.

${ }^{6} \mathrm{D}_{3}{ }^{8} \mathrm{G}^{\prime}{ }_{3}(0.20,0.60,1.00), 0.66,1.06,1.45,1.86,2.26$.

${ }^{6} \mathrm{D}_{3}-8 \mathrm{G}^{\prime}{ }_{4}(0.15,0.44,0.73), 0.63,0.93,1.22,1.51,1.80,2.09$.

${ }^{6} \mathrm{D}_{4}{ }^{-8} \mathrm{G}^{\prime}{ }_{3}(0.16,0.49,0.82), 0.76,1.09,1.42,1.75,2.08$, 2.42.

${ }^{6} \mathrm{D}_{4}{ }^{-8} \mathrm{G}^{\prime}{ }_{4}(0.11,0.33,0.55,0.78), 0.81,1.03,1.25,1.47,1.70,1.92,2.14$.

${ }^{6} \mathrm{D}_{4^{-}-8} \mathrm{G}_{5}^{\prime}{ }_{5}(0.09,0.26,0.43,0.61), 0.81,0.98,1.15,1.33,1.50,1.67,1.85,2.02$.

${ }^{6} \mathrm{D}_{5}-{ }^{8} \mathrm{G}_{4}{ }_{4}(0.09,0.29,0.48,0.67), 0.89,1.08,1.27,1.46,1.65,1.84,2.03$, 2.22.

${ }^{6} \mathrm{D}_{5}{ }^{8} \mathrm{G}^{\prime}{ }_{5}(0.07,0.21,0.35,0.49, \mathbf{0 . 6 4}), 0.92,1.06,1.20,1.34, \mathbf{1 . 4 8}, 1.62,1.77,1.91,2.05$.

${ }^{6} \mathrm{D}_{5}{ }^{-8} \mathrm{G}_{6}{ }_{6}(0.06,0.17,0.29,0.40,0.52), 0.92,1.04,1.15,1.27,1.38,1.50,1.61,1.73,1.84,1.96$.

${ }^{6} \mathrm{~F}_{2}-8 \mathrm{P}_{3}^{\prime}{ }_{3}(0.61,1.83), 0.46,1.68,2.90,4.12$.

${ }^{6} \mathrm{~F}_{3}-8 \mathrm{P}^{\prime}{ }_{3}(0.49,1.46,2.43),-0.14,+0.83,1.80,2.77,3.74$.

${ }^{6} \mathrm{~F}_{3}-8 \mathrm{P}^{\prime}{ }_{4}(0.31,0.93,1.55), 0.38,1.00,1.62,2.24,2.87,3.49$ 。

${ }^{6} \mathrm{~F}_{4}-8 \mathrm{P}^{\prime}{ }_{3}(0.44,1.33,2.22),-0.82,+0.06,0.95,1.84,2.73,3.62$.

${ }^{6} \mathrm{~F}_{4}-{ }^{8} \mathrm{P}_{4}^{\prime}(0.27,0.81,1.35,1.89), 0.05,0.59,1.13,1.6 \%, 2.21,2.75,3.29$.

${ }^{6} \mathrm{~F}_{4}{ }^{-8} \mathrm{P}_{5}^{\prime}(0.19,0.57,0.95,1.33), 0.44,0.82,1.21,1.59,1.97,2.35,2.73,3.11$.

${ }^{6} \mathrm{~F}_{5}-{ }^{-} \mathrm{P}_{4}^{\prime}(0.25,0.75,1.26,1.76),-0.32,+0.18,0.68,1.18,1.69,2.19,2.69,3.19$.

${ }^{6} \mathrm{~F}_{5}-8 \mathrm{P}_{5}^{\prime}(0.17,0.52,0.86,1.20,1.55), 0.23,0.58,0.92,1.26,1.61,1.95,2.29,2.64,2.98$.

${ }^{6} \mathrm{~F}_{6}-{ }^{-8} \mathrm{P}_{5}^{\prime}(0.16,0.48,0.81,1.13,1.46), 0.00,0.32,0.65,0.97,1.29,1.62,1.94,2.26,2.59,2.91$.

${ }^{6} \mathrm{~F}_{1}{ }^{-8} \mathrm{D}_{2}$ (1.73), $1.07,4.53$.

${ }^{6} \mathrm{~F}_{2}{ }^{8} \mathrm{D}_{2}(0.87,2.60), 0.20,1.93,3.67$.

${ }^{6} \mathrm{~F}_{2}-8 \mathrm{D}_{3}(0.49,1.48), 0.57,1.56,2.55,3.54$.

${ }^{6} \mathrm{~F}_{3}-{ }^{8} \mathrm{D}_{2}(0.74,2.23),-0.91,+0.57,2.06,3.54$.

${ }^{6} \mathrm{~F}_{3}-{ }^{-8} \mathrm{D}_{3}(0.37,1.11,1.56), 0.20,0.94,1.69,2.43,3.17$.

${ }^{6} \mathrm{~F}_{3}-{ }^{8} \mathrm{D}_{4}(0.25,0.74,1.24), 0.57,1.07,1.56,2.06,2.55,3.04$.

${ }^{6} \mathrm{~F}_{4}-{ }^{8} \mathrm{D}_{3}(0.33,0.99,1.65),-0.25,+0.41,1.07,1.72,2.38,3.04$.

${ }^{6} \mathrm{~F}_{4}{ }^{-8} \mathrm{D}_{4}(0.21,0.62,1.03,1.44), 0.36,0.78,1.19,1.60,2.02,2.43,2.84$.

${ }^{6} \mathrm{~F}_{4}{ }^{-8} \mathrm{D}_{5}(0.15,0.45,0.75,1.05), 0.65,0.95,1.25,1.55,1.85,2.15,2.45,2.75$.

${ }^{6} \mathrm{~F}_{5}-8 \mathrm{D}_{4}(0.19,0.56,0.93,1.31), 0.12,0.50,0.87,1.25,1.62,2.00,2.38,2.75$.

${ }^{6} \mathrm{~F}_{5}{ }^{-8} \mathrm{D}_{5}(0.13,0.39,0.66,0.92,1.18), 0.52,0.78,1.04,1.30,1.57,1.83,2.09,2.35,2.62$.

${ }^{6} \mathrm{~F}_{5}-8 \mathrm{D}_{6}(0.10,0.30,0.50,0.71,0.91), 0.73,0.93,1.13,1.34,1.54,1.74,1.94,2.14,2.34,2.54$.

${ }^{6} \mathrm{~F}_{6}-{ }^{8} \mathrm{D}_{5}(0.12,0.36,0.61,0.85,1.09), 0.36,0.61,0.85,1.09,1.33,1.58,1.82,2.06,2.30,2.54$.

${ }^{6} \mathrm{~F}_{6}-8 \mathrm{D}_{6}(0.09,0.27,0.45,0.64,0.82,1.00), 0.64,0.82,1.00,1.18,1.36,1.54,1.73,1.91,2.09,2.27,2.45$.

${ }^{6} \mathrm{~F}_{1}-8 \mathrm{~F}^{\prime}{ }_{1}(2.33), 1.67$.

${ }^{6} \mathrm{~F}_{1}-8 \mathrm{~F}^{\prime}{ }_{2}$ (1.33), $0.67,3.33$.

${ }^{6} \mathrm{~F}_{2}-8 \mathrm{~F}^{\prime}{ }_{1}(1.47),-\mathbf{0 . 4 0},+2.53$.

${ }^{6} \mathrm{~F}_{2}-8 \mathrm{~F}^{\prime}{ }_{2}(0.47,1.40), 0.60,1.53,2.47$.

${ }^{6} \mathrm{~F}_{2}-8 \mathrm{~F}^{\prime}{ }_{3}(0.32,0.97), 0.74,1.39,2.04$, 2.69.

${ }^{6} \mathrm{~F}_{3}-8 \mathrm{~F}^{\prime}{ }_{2}(0.31,1.03), 0.29,0.97,1.66,2.34$.

${ }^{6} \mathrm{~F}_{3}-8 \mathrm{~F}^{\prime}{ }_{3}(0.20,0.60,1.00), 0.71,1.11,1.51,1.91,2.31$.

${ }^{6} \mathrm{~F}_{3}-8 \mathrm{~F}_{4}^{\prime}(0.15,0.46,0.76), 0.86,1.16,1.47,1.77,2.07, \mathbf{2 . 3 8}$.

${ }^{6} \mathrm{~F}_{4}-8 \mathrm{~F}^{\prime}{ }_{3}(0.16,0.48,0.79), 0.60,0.92,1.24,1.55,1.87,2.19$

$6 \mathrm{~F}_{4}-8 \mathrm{~F}_{4}^{\prime}(0.11,0.33,0.55,0.78), 0.84,1.06,1.28,1.51,1.73,1.95,2.17$.

${ }^{6} \mathrm{~F}_{4}-8 \mathrm{~F}^{\prime}{ }_{5}(0.02,0.27,0.45,0.63), 0.95,1.13,1.31,1.49,1.67,1.84,2.02$, 2.20.

${ }^{6} \mathrm{~F}_{5}-8 \mathrm{~F}_{4}^{\prime}(9.09,0.28,0.46,0.65), 0.79,0.97,1.16,1.34,1.53,1.71,1.90,2.08$.

${ }^{6} \mathrm{~F}_{5}-8 \mathrm{~F}_{5}^{\prime}(0.07,0.21,0.35,0.49,0.64), 0.94,1.08,1.22,1.36,1.50,1.65,1.79,1.93,2.07$.

${ }^{6} \mathrm{~F}_{6}-8 \mathrm{~F}_{6}^{\prime}(0.06,0.18,0.30,0.41,0.53), 1.02,1.14,1.26,1.38,1.50,1.61,1.73,1.85,1.97,2.09$.

${ }^{6} \mathrm{~F}_{6}-{ }^{8} \mathrm{~F}_{5}{ }_{5}(0.06,0.18,0.30,0.42,0.55), 0.91,1.03,1.15,1.27,1.40,1.52,1.64,1.76,1.88,2.00$.

${ }^{6} \mathrm{~F}_{6}-8 \mathrm{~F}_{6}^{\prime}(0.05,0.15,0.24,0.34,0.44,0.54), 1.01,1.11,1.21,1.31,1.41,1.50,1.60,1.70,1.80,1.90,1.99$.

${ }^{6} \mathrm{~F}_{6}-{ }^{-8} \mathrm{~F}^{\prime} 7(0.94,0.13,0.21,0.29,0.38,0.46), 1.08,1.16,1.24,1.33,1.41,1.50,1.58,1.66,1.75,1.83,1.92,2.00$.

${ }^{6} \mathrm{~F}_{1^{-}}{ }^{8} \mathrm{G}_{1}(0.33), 1.00$.

${ }^{6} \mathrm{~F}_{1}-8 \mathrm{G}_{2}(0.80), 0.13,1.73$.

${ }^{6} \mathrm{~F}_{2}-8 \mathrm{G}_{1}(1.20),-0.13,+2.2 \%$.

${ }^{6} \mathrm{~F}_{2}-{ }^{8} \mathrm{G}_{2}(0.07,0.29), 0.87,1.00,1.13$.

${ }^{6} \mathrm{~F}_{2}{ }^{-8} \mathrm{G}_{3}(\mathbf{0 . 0 9}, 0.29), 0.97,1.16,1.35, \mathbf{1 . 5 4}$.

${ }^{6} \mathrm{~F}_{3}-{ }^{8} \mathrm{G}_{2}(0.19,0.57), 0.74,1.12,1.50,1.89$.

${ }^{6} \mathrm{~F}_{3}-{ }^{8} \mathrm{G}_{3}(0.03,0.09,0.14), 1.17,1.23,1.28,1.34,1.40$.

${ }^{6} \mathrm{~F}_{3}-{ }^{8} \mathrm{G}_{4}(0.03,0.08,0.13), 1.24,1.29,1.34,1.39,1.44,1.49$. 
TABLE 7.-Theoretical Zeeman effects (sextet-octet intersystem)-Continued

${ }^{6} \mathrm{~F}_{4}{ }^{-8} \mathrm{G}_{3}(0.07,0.21,0.35), 1.05,1.19,1.33,1.46,1.60,1.74$.

${ }^{6} \mathrm{~F}_{4^{-}}{ }^{8} \mathrm{G}_{4}(0.02,0.05,0.08,0.11), 1.29,1.32,1.35,1.38,1.41,1.45,1.48$.

${ }^{6} \mathrm{~F}_{4}-8 \mathrm{G}_{5}(0.01,0.03,0.04,0.06), 1.35,1.37,1.39,1.40,1.42,1.44,1.46,1.48$.

${ }^{6} \mathrm{~F}_{5}{ }^{-} \mathrm{G}_{4}(0.93,0.10,0.17,0.24), 1.19,1.26,1.33,1.40,1.47,1.54,1.51, \mathbf{1 . 6 8}$.

${ }^{6} \mathrm{~F}_{5}{ }^{-8} \mathrm{G}_{5}(0.01,0.03,0.05,0.07,0.09), 1.34,1.36,1.38,1.40,1.42,1.44,1.46,1.48,1.50$.

${ }^{6} \mathrm{~F}_{5^{-8}} \mathrm{G}_{6}(0.00,0.01,0.02,0.02,0.03), 1.41,1.42,1.43,1.43,1.44,1.44,1.45,1.46,1.46,1.47$.

${ }^{6} \mathrm{~F}_{6}-{ }^{8} \mathrm{G}_{5}(0.02,0.06,0.10,0.14,0.18), 1.27,1.31,1.35,1.40,1.44,1.48,1.52,1.56,1.50,1.64$.

${ }^{6} \mathrm{~F}_{6}{ }^{-}{ }^{-} \mathrm{G}_{6}(0.01,0.02,0.04,0.05,0.06,0.08), 1.38,1.39,1.41,1.42,1.43,1.45,1.46,1.47,1.49,1.50,1.52$.

${ }^{6} \mathrm{~F}_{6}{ }^{-8} \mathrm{G}_{7}(0.00,0.00,0.00,0.01,0.01,0.01), 1.45,1.45,1.45,1.45,1.45,1.46,1.46,1.46,1.46,1.46,1.47,1.4 \%$.

TABLE 8.-Theoretical Zeeman effects (triplet system)

[Landé $g$ values]

\begin{tabular}{|c|c|c|c|c|c|c|c|c|c|c|c|c|c|c|c|c|}
\hline 7 & 0 & 1 & 2 & 3 & 4 & 5 & 6 & 7 & 0 & 1 & 2 & 3 & 4 & 5 & 6 & 7 \\
\hline $\mathrm{S}$ & & $\frac{4}{2}$ & & & & & & & & 2.000 & & & & & & \\
\hline $\mathrm{P}$ & $\frac{0}{0}$ & $\frac{3}{2}$ & $\frac{9}{6}$ & & & & & & $\frac{0}{6}$ & 1. 500 & 1. 500 & & & & & \\
\hline $\mathrm{D}$ & & $\frac{1}{2}$ & $\frac{7}{6}$ & $\frac{18}{12}$ & & & & & & 0.500 & 1. 167 & 1. 333 & & & & \\
\hline $\mathrm{F}$ & & & $\frac{4}{6}$ & $\frac{13}{12}$ & $\frac{25}{20}$ & & & & & & 0.667 & 1. 083 & 1. 250 & & & \\
\hline G & & & & $\frac{9}{12}$ & $\frac{21}{20}$ & $\frac{38}{30}$ & & & & & & 0.750 & 1. 050 & 1. 200 & & \\
\hline H & & & & & $\frac{16}{20}$ & $\frac{31}{30}$ & $\frac{49}{42}$ & & & & & & 0.800 & 1. 033 & 1. 167 & \\
\hline I & & & & & & $\frac{25}{30}$ & $\frac{43}{42}$ & $\frac{64}{66}$ & & & & & & 0.833 & 1. 024 & 1.143 \\
\hline
\end{tabular}

${ }^{2} \mathrm{~S}_{1}{ }^{-\widehat{\jmath}} \mathrm{S}_{1}^{\prime}(0.00), 2.00$.

${ }^{3} \mathrm{~S}_{1}-3 \mathrm{P}_{0}(0.00), 2.00$.

${ }^{3} \mathrm{~S}_{1}-{ }^{3} \mathrm{P}_{1}(0.50), 1.50,2.00$.

${ }^{3} \mathrm{~S}_{1}-3 \mathrm{P}_{2}(0.00,0.50), 1.00,1.50,2.00$.

${ }^{3} \mathrm{~S}_{1}-{ }^{-2} \mathrm{D}_{1}^{\prime}(1.50), 0.50,2.00$.

${ }^{3} \mathrm{~S}_{1}{ }^{-3} \mathrm{D}_{2}^{\prime}{ }_{2}(\theta .00,0.83), 0.33,1.17,2.00$.

${ }^{3} \mathrm{~S}_{1}-{ }^{-3} \mathrm{~F}_{2}(0.00,1.33), 0.6 \%, 2.00$.

${ }^{3} \mathrm{P}_{0}{ }^{-3} \mathrm{P}_{1}^{\prime}={ }^{3} \mathrm{P}_{1}-{ }^{-3} \mathrm{P}_{0}^{\prime}={ }^{3} \mathrm{P}_{1}-{ }^{-3} \mathrm{P}_{1}^{\prime}={ }^{3} \mathrm{P}_{1}-{ }^{3} \mathrm{P}_{2}^{\prime}={ }^{3} \mathrm{P}_{2}-3 \mathrm{P}_{1}^{\prime}={ }^{3} \mathrm{P}_{2}-{ }^{3} \mathrm{P}_{2}^{\prime} \quad(0.00), 1.50$.

${ }^{3} \mathrm{P}_{0}-8 \mathrm{D}_{1}(0.00), 0.50$.

${ }^{3} \mathrm{P}_{1}{ }^{-3} \mathrm{D}_{1}(1.00), 0.50,1.50$.

${ }^{3} \mathrm{P}_{1}-{ }^{-3} \mathrm{D}_{2}(0.00,0.33), 0.33,1.17,1.50$.

${ }^{3} \mathrm{P}_{2}-{ }^{-3} \mathrm{D}_{1}(0.00,1.00), 0.50,1.50,2.50$.

${ }^{3} \mathrm{P}_{2}-{ }^{-3} \mathrm{D}_{2}(0.33,0.67), 0.83,1.17,1.50,1.83$.

${ }^{3} \mathrm{P}_{2}-{ }^{-3} \mathrm{D}_{3}(0.00,0.17,0.33), 1.00,1.17,1.33,1.50,1.67$.

${ }^{3} \mathrm{P}_{1}-{ }^{-} \mathrm{F}_{2}(0.00,0.83),-0.1 \%, 0.67,1.50$.

${ }^{3} \mathrm{P}_{2}-{ }^{-3} \mathrm{~F}_{2}(0.83,1.67),-0.17,0.67,1.50,2.33$.

${ }^{3} \mathrm{P}_{2}-3 \mathrm{~F}_{3}(0.00,0.42,0.83), 0.25,0.67,1.08,1.50,1.92$.

${ }^{3} \mathrm{P}_{2}{ }^{-3} \mathrm{G}_{3}(0.00,0.75,1.50),-0.75,0.00,0.75,1.50,2.25$.

${ }^{3} \mathrm{D}_{1}{ }^{-3} \mathrm{D}_{1}^{\prime}(0.00), 0.50$.

$\left.{ }^{3} \mathrm{D}_{1}-{ }^{-3} \mathrm{D}_{2}^{\prime} \mathrm{D}_{2} \mathrm{D}_{1}^{\prime}{ }_{1}\right\}(0.00,0.67), 0.50,1.17, \mathbf{1 . 8 3}$

${ }^{3} \mathrm{D}_{2}-{ }^{3} \mathrm{D}^{\prime}{ }_{2}(0.00), 1.17$.

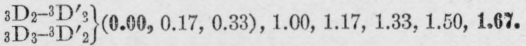

${ }^{3} \mathrm{D}_{3}-{ }^{3} \mathrm{D}_{3}^{\prime}{ }_{3}(0.00), 1.33$.

${ }^{3} \mathrm{D}_{1}-{ }^{3} \mathrm{~F}_{2}(0.00,0.17), 0.50,0.67,0.83$.

${ }^{3} \mathrm{D}_{2}-{ }^{-3} \mathrm{~F}_{2}(0.50,1.00), 0.17, \mathbf{0 . 6 7}, \mathbf{1 . 1 7}, 1.67$.

${ }^{3} \mathrm{D}_{3}{ }^{-3} \mathrm{~F}_{2}(0.00,0.67,1.33), 0.00,0.67,1.33,2.00,2.67$.

${ }^{3} \mathrm{D}_{2}-{ }^{3} \mathrm{~F}_{3}(0.00,0.08,0.17), 0.92,1.00,1.08,1.17,1.25$.

${ }^{3} \mathrm{D}_{3}{ }^{-3} \mathrm{~F}_{3}(0.25,0.50,0.75), 0.58,0.83,1.08,1.33,1.58,1.83$.

${ }^{3} \mathrm{D}_{3}{ }^{3} \mathrm{~F}_{4}(0.00,0.08,0.17,0.25) .1 .00,1.08,1.17,1.25,1.33,1.42 .1 .50$. 
TABLE 8.-Theoretical Zeeman effects (triplet system)-Continued

${ }^{3} \mathrm{D}_{2}{ }^{-3} \mathrm{G}_{3}{ }_{3}(0.00,0.42,0.83),-0.08,+0.33,0.75,1.17,1.58$.

${ }^{3} \mathrm{D}_{3}{ }^{-3} \mathrm{G}^{\prime}{ }_{3}(0.58,1.17,1.75),-0.42,+0.17,0.75,1.33,1.92,2.50$.

${ }^{8} \mathrm{D}_{3}-3 \mathrm{G}_{4}^{\prime}(0.00,0.28,0.57,0.85), 0.20,0.48,0.77,1.05,1.33,1.62,1.90$.

${ }^{3} \mathrm{D}_{3}{ }^{3} \mathrm{H}_{4}(0.00,0.53,1.07,1.60),-0.80,-0.27,+0.27,0.80,1.33,1.87,2.40$.

${ }^{3} \mathrm{~F}_{2}-3 \mathrm{~F}^{\prime}{ }_{2}(0.00), 0.67$.

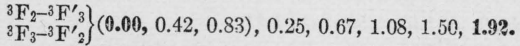

${ }^{3} \mathrm{~F}_{3}-{ }^{3} \mathrm{~F}^{\prime}{ }_{3}(0.00), 1.08$.

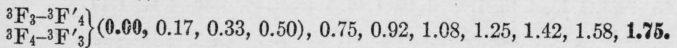

${ }^{3} \mathrm{~F}_{4}-3 \mathrm{~F}^{\prime}{ }_{4}(0.00), 1.25$.

${ }^{3} \mathrm{~F}_{2}-{ }^{3} \mathrm{G}_{3}(0.00,0.08,0.17), 0.58,0.67,0.75,0.83,0.92$.

${ }^{8} \mathrm{~F}_{3}{ }^{-3} \mathrm{G}_{3}(0.33,0.67,1.00), 0.08,0.42,0.75,1.08,1.42,1.75$.

${ }^{3} \mathrm{~F}_{3}-{ }^{-3} \mathrm{G}_{4}(0.00,0.03,0.07,0.10), 0.95,0.98,1.02,1.05,1.08,1.12,1.15$.

${ }^{3} \mathrm{~F}_{4}{ }^{-3} \mathrm{G}_{3}(0.00,0.50,1.00,1.50),-0.25,+0.25,0.75,1.25,1.75,2.25,2.75$.

${ }^{3} \mathrm{~F}_{4}{ }^{-3} \mathrm{G}_{4}(0.20,0.40,0.60,0.80), 0.45,0.65,0.85,1.05,1.25,1.45,1.65,1.85$.

${ }^{3} \mathrm{~F}_{4}-{ }^{3} \mathrm{G}_{5}(\mathbf{0 . 0 0}, 0.05,0.10,0.15,0.20), 1.00,1.05,1.10,1.15,1.20,1.25,1.30,1.35,1.40$.

${ }^{3} \mathrm{~F}_{3}-{ }^{-3} \mathrm{H}_{4}^{\prime}(0.00,0.28,0.57,0.85),-0.05,+0.23,0.52,0.80,1.08,1.37,1.65$.

${ }^{3} \mathrm{~F}_{4}-{ }^{3} \mathrm{H}^{\prime}{ }_{4}(0.45,0.90,1.35,1.80),-0.55,-0.10,+0.35,0.80,1.25,1.70,2.15,2.60$.

${ }^{3} \mathrm{~F}_{4}{ }^{-3} \mathrm{H}_{5}^{\prime}(0.00,0.22,0.43,0.65,0.87), 0.17,0.38,0.60,0.82,1.03,1.25,1.47,1.68,1.90$.

${ }^{3} \mathrm{~F}_{4}-{ }^{3} \mathrm{I}_{5}(\mathbf{0 . 0 0}, 0.42,0.83,1.25,1.67),-0.83,-0.42,0.00,0.42,0.83,1.25,1.67,2.08,2.50$.

${ }^{8} \mathrm{G}_{3}-3 \mathrm{G}_{3}{ }_{3}(0.00), 0.75$.

$\left.{ }^{3} \mathrm{G}_{3}{ }^{-3} \mathrm{G}^{\prime}{ }^{3} \mathrm{G}_{4-3}^{-3} \mathrm{G}_{3}^{\prime}{ }_{3}\right\}(0.00,0.30,0.60,0.90), 0.15,0.45,0.75,1.05,1.35,1.65,1.95$.

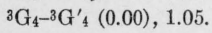

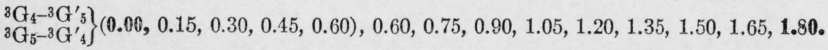

${ }^{3} \mathrm{G}_{5}-3 \mathrm{G}^{\prime}{ }_{5}(0.00), 1.20$.

${ }^{3} \mathrm{G}_{3}-{ }^{3} \mathrm{H}_{4}(\mathbf{0 . 0 0}, 0.05,0.10,0.15), 0.65,0.70,0.75,0.80,0.85,0.90,0.95$.

${ }^{8} \mathrm{G}_{4}{ }^{-3} \mathrm{H}_{4}(0.25,0.50,0.75, \mathbf{1 . 0 0}), 0.05,0.30,0.55,0.80,1.05,1.30,1.55,1.80$.

${ }^{3} \mathrm{G}_{5}-{ }^{3} \mathrm{H}_{4}(0.00,0.40,0.80,1.20,1.60), 0.00,0.40,0.80,1.20,1.60,2.00,2.40,2.80$.

${ }^{8} \mathrm{G}_{4-3}{ }^{-3} \mathrm{H}_{5}(\theta .00,0.02,0.03,0.05,0.07), 0.97,0.98,1.00,1.02,1.03,1.05,1.07,1.08,1.10$.

${ }^{8} \mathrm{G}_{5}-{ }^{3} \mathrm{H}_{5}(0.17,0.33,0.50,0.67,0.83), 0.37,0.53,0.70,0.87,1.03,1.20,1.37,1.53,1.70,1.87$.

${ }^{3} \mathrm{G}_{5}{ }^{-3} \mathrm{H}_{6}(0.00,0.03,0.07,0.10,0.13,0.17), 1.00,1.03,1.07,1.10,1.13,1.17,1.20,1.23,1.27,1.30,1.33$.

${ }^{8} \mathrm{G}_{4}-3 \mathrm{I}_{5}^{\prime}(0.00,0.22,0.43,0.65,0.87),-0.03,+0.18,0.40,0.62,0.83,1.05,1.27,1.48,1.70$.

${ }^{3} \mathrm{G}_{5}-{ }^{-3} \mathrm{I}_{5}{ }_{5}(0.37,0.73,1.10,1.47,1.83),-0.63,-0.27,+0.10,0.47,0.83,1.20,1.57,1.93,2.30,2.67$.

${ }^{3} \mathrm{G}_{5}-{ }^{-3} \mathrm{I}_{6}{ }_{6}(0.00,0.18,0.35,0.53,0.70,0.88), 0.14,0.32,0.50,0.67,0.85,1.02,1.20,1.38,1.55,1.73,1.90$.

${ }^{3} \mathrm{H}_{4}-3 \mathrm{H}^{\prime}{ }_{4}(0.00), 0.80$.

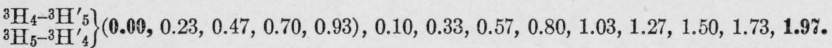

${ }^{3} \mathrm{H}_{5}{ }^{3} \mathrm{H}_{5}^{\prime}(0.00), 1.03$.

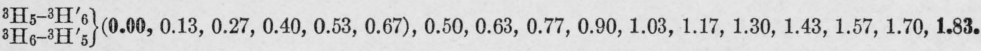
${ }^{3} \mathrm{H}_{0}-3 \mathrm{H}_{6}^{\prime}(0.00), 1.17$.

${ }^{3} \mathrm{H}_{4}-{ }^{3} \mathrm{I}_{5}(\mathbf{0 . 0 9}, 0.03,0.07,0.10,0.13), 0.70,0.73,0.77,0.80,0.83,0.87,0.90,0.93,0.9 \%$.

${ }^{3} \mathrm{H}_{5^{-3} \mathrm{I}_{5}(0.20,0.40,0.60,0.80,1.00)}, 0.03,0.23,0.43,0.63,0.83,1.03,1.23,1.43,1.63,1.83$.

${ }^{3} \mathrm{H}_{5}-3 \mathrm{I}_{6}(\mathbf{0 . 0 0}, 0.01,0.02,0.03,0.04,0.05), 0.98,0.99,1.00,1.01,1.02,1.03,1.04,1.05,1.06,1.07$.

${ }^{3} \mathrm{H}_{6}{ }^{-3} \mathrm{I}_{5}(\mathbf{0 . 0 0}, 0.33,0.67,1.00,1.33,1.67),-0.50,-0.17,+0.17,0.50,0.83,1.17,1.50,1.83,2.17,2.50$, 2.83.

${ }^{3} \mathrm{H}_{6}-{ }^{3} \mathrm{I}_{6}(0.14,0.29,0.43,0.57,0.71,0.86), 0.31,0.45,0.60,0.74,0.88,1.02,1.17,1.31,1.45,1.60,1.74,1.88$.

${ }^{3} \mathrm{H}_{6}{ }^{-3} \mathrm{I}_{7}(0.00,0.02,0.05,0.07,0.10,0.12,0.14), 1.00,1.02,1.05,1.07,1.10,1.12,1.14,1.17,1.19,1.21,1.24,1.26,1.29$.

${ }^{3} \mathrm{I}_{5}-{ }^{-3} \mathrm{I}_{5}{ }_{5}(0.00), 0.83$.

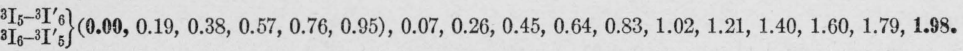

${ }^{3} \mathrm{I}_{6}-3 \mathrm{I}_{6}^{\prime}(0.00), 1.02$.

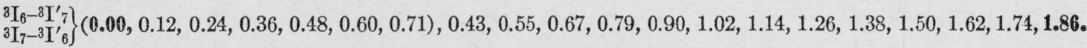
${ }^{3} \mathrm{I}_{7}-{ }^{-3} \mathrm{I}_{7}^{\prime}(0.00), 1.14$. 
TABLE 9.-Theoretical Zeeman effects (quintet system)

[Landé $g$ values] 
TABLE 9.-Theoretical Zeeman effects (quintet system) - Continued

${ }^{8} \mathrm{~S}_{2}-8 \mathrm{~S}_{2}^{\prime}(0.00), 2.00$.

${ }^{8} \mathrm{~S}_{2}-{ }^{-5} \mathrm{P}_{1}(0.00,0.50), 1.50,2.00,2.50$.

${ }^{5} \mathrm{~S}_{2}-5 \mathrm{P}_{2}(0.17,0.33), 1.67,1.83,2.00,2.17$.

${ }^{5} \mathrm{~S}_{2}-\mathrm{P}_{3} \mathrm{P}_{3}(\mathbf{0 . 0 0}, 0.33,0.67), \mathbf{1 . 0 0}, 1.33,1.67,2.00,2.33$.

${ }^{5} \mathrm{~S}_{2}-{ }^{-5} \mathrm{D}^{\prime}{ }_{1}(0.00,0.50), 1.50,2.00, \mathbf{2 . 5 0} 0$.

${ }^{8} \mathrm{~S}_{2}-5 \mathrm{D}^{\prime}{ }_{2}(0.50,1.00), 1.00,1.50,2.00,2.50$.

${ }^{8} \mathrm{~S}_{2}-{ }^{-6} \mathrm{D}_{3}^{\prime}(0.00,0.50,1.00), 0.50,1.00,1.50,2.00,2.50$.

${ }^{6} \mathrm{~S}_{2}-5 \mathrm{~F}_{1}(0.00,2.00), 0.00,2.00,4.00$.

${ }^{8} \mathrm{~S}_{2}-5 \mathrm{~F}_{2}(1.00,2.00), 0.00,1.00,2.00,3.00$.

${ }^{8} \mathrm{~S}_{2}-5 \mathrm{~F}_{3}(0.00,0.75,1.50),-\mathbf{0 . 2 5},+0.50,1.25,2.00,2.75$.

${ }^{8} \mathrm{P}_{1}-5 \mathrm{P}_{1}^{\prime}(0.00), 2.50$.

$\left.{ }^{5} \mathrm{P}_{1}-5 \mathrm{P}_{2}^{\prime} \mathrm{P}_{2} \mathrm{P}_{2} \mathrm{P}_{1}\right\}^{8} \mathrm{P}_{2}(0.00,0.67,) \mathbf{1 . 1 7}, 1.83,2.50$.

${ }^{8} \mathrm{P}_{2}-5 \mathrm{P}_{2}^{\prime}{ }_{2}(0.00), 1.83$.

$\left.{ }_{6}^{8} \mathrm{P}_{2}-5 \mathrm{P}_{3}-5 \mathrm{P}_{2}^{\prime}{ }_{2}^{\prime}\right\}(0.00,0.17,0.33), 1.33,1.50,1.67,1.83,2.00$.

${ }^{5} \mathrm{P}_{3}-5 \mathrm{P}_{3}^{\prime}(0.00), 1.67$

${ }^{5} \mathrm{P}_{1}-5 \mathrm{D}_{0}(0.00), 2.50$.

${ }^{5} \mathrm{P}_{1}{ }^{-5} \mathrm{D}_{1}(1.00), 1.50,2.50$.

${ }^{5} \mathrm{P}_{1}{ }^{5} \mathrm{D}_{2}(\mathbf{0 . 0 0}, 1.00), \mathbf{0 . 5 0}, 1.50,2.50$.

${ }^{5} \mathrm{P}_{2}-{ }^{5} \mathrm{D}_{1}(\mathbf{0 . 0 0}, 0.33), 1.50,1.83, \mathbf{2 . 1 7}$.

${ }^{5} \mathrm{P}_{2}-5 \mathrm{D}_{2}(0.33,0.67), 1.17,1.50,1.83,2.17$.

${ }^{5} \mathrm{P}_{2}-5 \mathrm{D}_{3}(0.00,0.33,0.67), 0.83,1.17,1.50,1.83,2.17$.

${ }^{b} \mathrm{P}_{3}-5 \mathrm{D}_{2}(\mathbf{0 . 0 0}, 0.17,0.33), 1.33,1.50,1.67,1.83, \mathbf{2 . 0 0}$.

${ }^{5} \mathrm{P}_{3}{ }^{-5} \mathrm{D}_{3}(0.17,0.33,0.50), 1.17,1.33, \mathbf{1 . 5 0}, \mathbf{1 . 6 7}, 1.83,2.00$.

${ }^{6} \mathrm{P}_{3}{ }^{-5} \mathrm{D}_{4}(0.00,0.17,0.33,0.50), 1.80,1.17,1.33,1.50,1.67,1.83,2.00$.

${ }^{\circ} \mathrm{P}_{1}-5 \mathrm{~F}^{\prime}{ }_{1}(2.50), 0.00,2.50$.

${ }^{3} \dot{\mathrm{P}}_{1}{ }^{-5} \mathrm{~F}^{\prime}{ }_{2}(0.00,1.50),-0.50,+1.00,2.50$.

${ }^{5} \mathrm{P}_{2}-5 \mathrm{~F}^{\prime}{ }_{1}(0.00,1.83), 0.00,1.83, \mathbf{3 . 6 7}$.

${ }^{5} \mathrm{P}_{2}{ }^{5} \mathrm{~F}^{\prime}{ }_{2}(0.83,1.67), 0.17,1.00,1.83,2.67$.

${ }^{8} \mathrm{P}_{2}-5 \mathrm{~F}^{\prime}{ }_{3}(0.00,0.58,1.17), 0.08,0.67,1.25,1.83,2.42$.

${ }^{5} \mathrm{P}_{3}-5 \mathrm{~F}^{\prime}{ }_{2}(0.00,0.67,1.33), 0.33,1.00,1.67,2.33,3.00$.

${ }^{5} \mathrm{P}_{3}-5 \mathrm{~F}^{\prime}{ }_{3}(0.42,0.83,1.25), 0.42,0.83,1.25,1.67,2.08,2.50$.

${ }^{5} \mathrm{P}_{3}-{ }^{-5} \mathrm{~F}_{4}^{\prime}(0.00,0.32,0.63,0.95), 0.40,0.72,1.03,1.35,1.67,1.98,2.30$.

${ }^{8} \mathrm{P}_{1^{-5}} \mathrm{G}_{2}(\mathbf{0 . 0 0}, 2.17),-\mathbf{1 . 8 3},+0.33,2.50$.

${ }^{5} \mathrm{P}_{2}-5 \mathrm{G}_{2}(1.50,3.00),-1.17,+0.33,1.83,3.33$.

${ }^{5} \mathrm{P}_{2}-5 \mathrm{G}_{3}(0.00,0.92,1.83),-0.92,0.00,0.92,1.83,2.75$.

${ }^{5} \mathrm{P}_{3}{ }^{-5} \mathrm{G}_{2}(0.00,1.33,2.67),-1.00,+0.33,1.67,3.00$, 4.33.

${ }^{8} \mathrm{P}_{3}{ }^{-5} \mathrm{G}_{3}(0.75,1.50,2.25),-0.58,+0.17,0.92,1.67,2.42,3.17$.

${ }^{8} \mathrm{P}_{3}-5 \mathrm{G}_{4}(0.00,0.52,1.03,1.55),-0.40,+0.12,0.63,1.15,1.67,2.18,2.70$.

${ }^{5} \mathrm{D}_{1}-5 \mathrm{D}^{\prime}{ }_{0}={ }^{5} \mathrm{D}_{1}-{ }^{5} \mathrm{D}_{1}^{\prime}{ }_{1}={ }^{5} \mathrm{D}_{1}-{ }^{5} \mathrm{D}_{2}^{\prime}=\ldots={ }^{5} \mathrm{D}_{4}-{ }^{5} \mathrm{D}_{4}^{\prime}(0.00), 1.50$.

${ }^{5} \mathrm{D}_{0}{ }^{-5} \mathrm{~F}_{1}(0.00), 0.00$, unaffected.

${ }^{3} \mathrm{D}_{1}-5 \mathrm{~F}_{1}(1.50), 0.00,1.50$.

${ }^{5} \mathrm{D}_{1}{ }^{-5} \mathrm{~F}_{2}(\mathbf{0 . 0 0}, 0.50), \mathbf{0 . 5 0}, 1.00,1.50$.

${ }^{5} \mathrm{D}_{2}-5 \mathrm{~F}_{1}(0.00,1.50), 0.00,1.50,2.00$.

${ }^{5} \mathrm{D}_{2}-{ }^{5} \mathrm{~F}_{2}(0.50,1.00), 0.50,1.00,1.50,2.00$.

${ }^{5} \mathrm{D}_{2}-{ }^{5} \mathrm{~F}_{3}(0.00,0.25,0.50), 0.75,1.00,1.25,1.50,1.75$.

${ }^{5} \mathrm{D}_{3}{ }^{-5} \mathrm{~F}_{2}(0.00,0.50,1.00), 0.50,1.00,1.50,2.00, \mathbf{2 . 5 0}$.

${ }^{5} \mathrm{D}_{3}{ }^{-5} \mathrm{~F}_{3}(0.25,0.50,0.75), 0.75,1.00,1.25,1.50,1.75,2.00$.

${ }^{5} \mathrm{D}_{3}{ }^{-5} \mathrm{~F}_{4}(0.00,0.15,0.30,0.45), 0.90,1.05,1.20,1.35,1.50,1.65,1.80$.

${ }^{5} \mathrm{D}_{4}{ }^{-5} \mathrm{~F}_{3}(0.00,0.25,0.50,0.75), 0.75,1.00,1.25,1.50,1.75,2.00,2.25$.

${ }^{5} \mathrm{D}_{4}{ }^{-5} \mathrm{~F}_{4}(0.15,0.30,0.45,0.60), 0.90,1.05,1.20, \mathbf{1 . 3 5}, \mathbf{1 . 5 0}, 1.65,1.80,1.95$.

${ }^{8} \mathrm{D}_{4}{ }^{-5} \mathrm{~F}_{5}(0.00,0.10,0.20,0.30,0.40), 1.00,1.10,1.20,1.30,1.40,1.50,1.60,1.70,1.80$.

${ }^{\circ} \mathrm{D}_{1}{ }^{-5} \mathrm{G}_{2}^{\prime}(0.00,1.17),-0.83,+0.33,1.50$.

${ }^{5} \mathrm{D}_{2}{ }^{-5} \mathrm{G}_{2}^{\prime}(1.17,2.33),-0.83,+0.33,1.50,2.67$.

${ }^{5} \mathrm{D}_{2}-5 \mathrm{G}^{\prime}{ }_{3}(0.00,0.58,1.17),-0.25,+0.33,0.92,1.50,2.08$.

${ }^{5} \mathrm{D}_{3}{ }^{-5} \mathrm{G}_{2}{ }_{2}(0.00,1.17,2.33),-0.83,+0.33,1.50,2.67,3.83$.

${ }^{5} \mathrm{D}_{3}-5 \mathrm{G}^{\prime}{ }_{3}(0.58,1.17,1.75),-0.25,+0.33,0.92,1.50,2.08,2.67$.

${ }^{5} \mathrm{D}_{3}{ }^{-5} \mathrm{G}^{\prime}{ }_{4}(0.00,0.35,0.70,1.05), 0.10,0.45,0.80,1.15,1.50,1.85,2.20$.

${ }^{5} \mathrm{D}_{4}{ }^{-5} \mathrm{G}^{\prime}{ }_{3}(0.00,0.58,1.17,1.75),-0.25,+0.33,0.92,1.50,2.08,2.67,3.25$.

${ }^{5} \mathrm{D}_{4^{-5}} \mathrm{G}^{\prime}{ }_{4}(0.35,0.70,1.05,1.40), 0.10,0.45,0.80,1.15,1.50,1.85,2.20,2.55$.

${ }^{5} \mathrm{D}_{4}{ }^{-5} \mathrm{G}^{\prime}{ }_{5}(0.00,0.23,0.47,0.70,0.93), 0.33,0.57,0.80,1.03,1.27,1.50,1.73,1.97,2.20$. 
TABLE 9.-Theoretical Zeeman effects (quintet system)-Continued

${ }^{5} \mathrm{D}_{2}-5 \mathrm{H}_{3}(\mathbf{0 . 0 0}, 1.00,2.00),-1.50,-0.50,+0.50,1.50,2.50$.

${ }^{5} \mathrm{D}_{3}{ }^{-5} \mathrm{H}_{3}(1.00,2.00,3.00),-1.50,-0.50,+0.50,+1.50,2.50,3.50$.

${ }^{5} \mathrm{D}_{3}{ }^{-5} \mathrm{H}_{4}(0.00,0.60,1.20,1.80),-0.80,-0.30,+0.30,0.90,1.50,2.10,$,2.70 .

${ }^{5} \mathrm{D}_{4^{-5}} \mathrm{H}_{3}(0.00,1.00,2.00,3.00),-1.50,-0.50,+0.50,1.50,2.50,3.50,4.50$.

${ }^{5} \mathrm{D}_{4}{ }^{-5} \mathrm{H}_{4}(0.60,1.20,1.80,2.40),-0.90,-0.30,+0.30,0.90,1.50,2.10,2.70,3.30$.

${ }^{5} \mathrm{D}_{4^{-5}} \mathrm{H}_{5}(0.00,0.40,0.80,1.20,1.60),-0.50,-0.10,+0.30,0.70,1.10,1.50,1.90,2.30,2.70$.

${ }^{8} \mathrm{~F}_{1}-5 \mathrm{~F}_{1}{ }_{1}(0.00), 0.00$ unaffected.

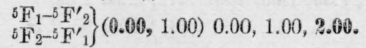

${ }^{8} \mathrm{~F}_{2}-5 \mathrm{~F}^{\prime}{ }_{2}(0.00), 1.00$.

$\left.{ }^{5} \mathrm{~F}_{2}-5 \mathrm{~F}_{3}-5 \mathrm{~F}^{\prime}{ }^{\prime}{ }_{2}{ }^{3}\right\}(0.00,0.25,0.50), 0.75,1.00,1.25,1.50,1.75$.

${ }^{6} \mathrm{~F}_{3}-5 \mathrm{~F}^{\prime}{ }_{3}(0.00), 1.25$.

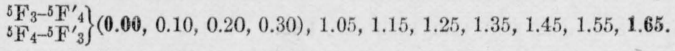

${ }^{5} \mathrm{~F}_{4}-5 \mathrm{~F}^{\prime}{ }_{4}(0.00), 1.35$.

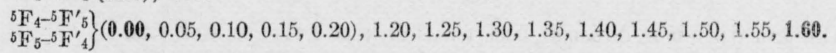

${ }^{5} \mathrm{~F}_{5}{ }^{-5} \mathrm{~F}_{5}^{\prime}(0.00), 1.40$.

${ }^{5} \mathrm{~F}_{1^{-5}} \mathrm{G}_{2}(\mathbf{0 . 0 0}, 0.33), 0.00,0.33, \mathbf{0 . 6 7}$.

${ }^{5} \mathrm{~F}_{2}-{ }^{-5} \mathrm{G}_{2}(0.67,1.33), 0.33,1.00,1.67$.

${ }^{5} \mathrm{~F}_{2^{-5}} \mathrm{G}_{3}(0.00,0.08,0.17), 0.75,0.83,0.92,1.00,1.08$.

${ }^{5} \mathrm{~F}_{3}{ }^{-5} \mathrm{G}_{2}(\mathbf{0 . 0 0}, 0.92,1.83),-0.58,+0.33,1.25,2.17,3.08$.

${ }^{5} \mathrm{~F}_{3}{ }^{-5} \mathrm{G}_{3}(0.33,0.67,1.00), 0.25,0.58,0.92,1.25,1.58,1.92$.

${ }^{5} \mathrm{~F}_{3}{ }^{-5} \mathrm{G}_{4}(0.00,0.10,0.20,0.30), 0.85,0.95,1.05,1.15,1.25,1.35,1.45$.

${ }^{5} \mathrm{~F}_{4}-5 \mathrm{G}_{3}(0.00,0.43,0.87,1.30), 0.05,0.48,0.92,1.35,1.78,2.22,2.65$.

${ }^{5} \mathrm{~F}_{4}{ }^{-5} \mathrm{G}_{4}(0.20,0.40,0.60,0.80), 0.55,0.75,0.95,1.15,1.35,1.55,1.75,1.95$.

${ }^{5} \mathrm{~F}_{4}{ }^{-5} \mathrm{G}_{5}(\mathbf{0 . 6 0}, 0.08,0.17,0.25,0.33) .0 .93,1.02,1.10,1.18,1.27,1.35,1.43,1.52,1.60$.

${ }^{5} \mathrm{~F}_{5^{-5}} \mathrm{G}_{4}(\mathbf{0 . 0 0}, 0.25,0.50,0.75,1.00), 0.40,0.65,0.90,1.15,1.40,1.65,1.90,2.15,2.40$.

${ }^{5} \mathrm{~F}_{5}{ }^{-5} \mathrm{G}_{5}(0.13,0.27,0.40,0.53, \mathbf{0 . 6 7}), 0.73,0.87,1.00,1.13, \mathbf{1 . 2 7}, \mathbf{1 . 4 0}, 1.53,1.67,1.80,1.93$.

${ }^{5} \mathrm{~F}_{5}{ }^{-5} \mathrm{G}_{6}(\mathbf{0 . 0 0}, 0.07,0.13,0.20,0.27,0.33), 1.00,1.07,1.13,1.20,1.27,1.33,1.40,1.47,1.53,1.60,1.67$.

${ }^{5} \mathrm{~F}_{2}-5 \mathrm{H}^{\prime}{ }_{3}(\mathbf{0 . 0 0}, 0.50,1.00),-0.50,0.00,0.50,1.00,1.50$.

${ }^{5} \mathrm{~F}_{3}{ }^{-5} \mathrm{H}_{3}^{\prime}(0.75,1.50,2.25),-1.00,-0.25,+0.50,1.25,2.00,2.75$.

${ }^{5} \mathrm{~F}_{3}-5 \mathrm{H}^{\prime}{ }_{4}(0.00,0.35,0.70,1.05),-0.15,+0.20,0.55,0.90,1.25,1.60,1.95$.

${ }^{5} \mathrm{~F}_{4}{ }^{-5} \mathrm{H}_{3}{ }_{3}(0.00,0.85,1.70,2.55),-1.20,-0.35,+0.50,1.35,2.20,3.05,3.90$.

${ }^{5} \mathrm{~F}_{4}-{ }^{-5} \mathrm{H}_{4}^{\prime}(0.45,0.90,1.35,1.80),-0.45,0.00,0.45,0.90,1.35,1.80,2.25,2.70$.

${ }^{3} \mathrm{~F}_{4}{ }^{-5} \mathrm{H}_{5}{ }_{5}(\mathbf{0 . 0 0}, 0.25,0.50,0.75,1.00), 0.10,0.35,0.60,0.85,1.10,1.35,1.60,1.85,2.10$.

${ }^{5} \mathrm{~F}_{5}-5 \mathrm{H}^{\prime}{ }_{4}(0.00,0.50,1.00,1.50,2.00),-0.60,-0.10,+0.40,0.90,1.40,1.90,2.40,2.90,3.40$.

${ }^{5} \mathrm{~F}_{5}{ }^{-5} \mathrm{H}^{\prime}{ }_{5}(0.30,0.60,0.90,1.20,1.50),-0.10,+0.20,0.50,0.80,1.10,1.40,1.70,2.00,2.30,2.60$.

${ }^{5} \mathrm{~F}_{5}-{ }^{-5} \mathrm{H}_{6}{ }_{6}(0.00,0.19,0.37,0.56,0.74,0.93), 0.29,0.47,0.66,0.84,1.03,1.21,1.40,1.59,1.77,1.96,2.14$.

${ }^{5} \mathrm{G}_{2}-{ }^{5} \mathrm{G}_{2}{ }_{2}(0.00), 0.33$.

$\left.{ }^{5} \mathrm{G}_{2} \mathrm{G}_{3}^{-5} \mathrm{G}^{5} \mathrm{G}_{2}^{\prime}{ }_{2}^{\prime}\right\}(0.00,0.58,1.17),-0.25,+0.33,0.92,1.50,2.08$.

${ }^{8} \mathrm{G}_{3}-5 \mathrm{G}^{\prime}{ }_{3}(0.00), 0.92$.

$\left.{ }^{{ }^{8} \mathrm{G}_{3} \mathrm{G}_{4-5}^{-5} \mathrm{G}^{\prime}{ }_{4}{ }_{3}{ }_{3}}\right\}(0.00,0.23,0.47,0.70), 0.45,0.68,0.92,1.15,1.38,1.62 .1 .85$.

${ }^{8} \mathrm{G}_{4}{ }^{-5} \mathrm{G}_{4}^{\prime}(0.00), 1.15$.

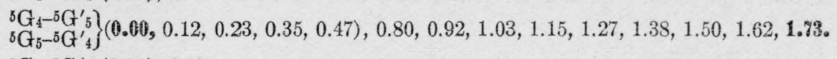

${ }^{6} \mathrm{G}_{5}-{ }^{5} \mathrm{G}^{\prime}{ }_{5}(0.00), 1.27$.

$\left.{ }^{{ }^{3} \mathrm{G}_{5^{-5}} \mathrm{G}_{6^{-5}} \mathrm{G}^{\prime}{ }^{\prime}{ }_{5}}\right\}(0.00,0.07,0.13,0.20,0.27,0.33), 1.00,1.07,1.13,1.20,1.27,1.33,1.40,1.47,1.53,1.60,1.67$.

${ }^{5} \mathrm{G}_{6}{ }^{-5} \mathrm{G}^{\prime}{ }_{6}(0.00), 1.33$.

${ }^{5} \mathrm{G}_{2}-{ }^{-5} \mathrm{H}_{3}(0.00,0.17,0.33), 0.17,0.33,0.50,0.67,0.83$.

${ }^{8} \mathrm{G}_{3}-{ }^{-5} \mathrm{H}_{3}(0.42,0.83,1.25),-0.33,+0.08,0.50,0.92,1.33,1.75$.

${ }^{5} \mathrm{G}_{3}-{ }^{5} \mathrm{H}_{4}(0.00,0.02,0.03,0.05), 0.85 .0 .87,0.88,0.90,0.92,0.93,0.95$.

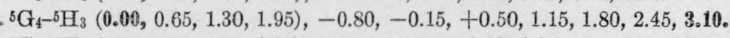

${ }^{5} \mathrm{G}_{4-5} \mathrm{H}_{4}(0.25,0.50,0.75,1.00), 0.15,0.40,0.65,0.90,1.15,1.40,1.65,1.90$.

${ }^{5} \mathrm{G}_{4}-5 \mathrm{H}_{5}(0.00,0.05,0.10,0.15,0.20), 0.90,0.95,1.00,1.05,1.10,1.15,1.20,1.25,1.30$.

${ }^{5} \mathrm{G}_{5}-{ }^{5} \mathrm{H}_{4}(0.00,0.37,0.73,1.10,1.47),-0.20,+0.17,0.53,0.90,1.27,1.63,2.00,2.37,2.73$.

${ }^{5} \mathrm{G}_{5}-{ }^{-5} \mathrm{H}_{5}(0.17,0.33,0.50,0.67,0.83), 0.43,0.60,0.77,0.93,1.10,1.27,1.43,1.60,1.77,1.93$.

${ }^{5} \mathrm{G}_{5}-{ }^{5} \mathrm{H}_{6}(0.00,0.05,0.10,0.16,0.21,0.26), 0.95,1.00,1.06,1.11,1.16,1.21,1.27,1.32,1.37,1.42,1.48$.

${ }^{5} \mathrm{G}_{6}-{ }^{-5} \mathrm{H}_{5}(0.00,0.23,0.47,0.70,0.93,1.17), 0.17,0.40,0.63,0.87,1.10,1.33,1.57,1.80,2.03,2.27,2.50$.

${ }^{5} \mathrm{G}_{6}{ }^{-5} \mathrm{H}_{0}(0.12,0.24,0.36,0.48,0.60,0.71), 0.62,0.74,0.86,0.98,1.10,1.22,1.33,1.45,1.57,1.69,1.81,1.93$.

${ }^{5} \mathrm{G}_{6}{ }^{-5} \mathrm{H}_{7}(0.00,0.05,0.10,0.14,0.19,0.24,0.29), \mathbf{1 . 0 0}, 1.05,1.10,1.14,1.19,1.24,1.29,1.33,1.38,1.43,1.48,1.52,1.57$. 
TABLE 9.-Theoretical Zeeman effects (quintet system)-Continued

${ }^{5} \mathrm{G}_{3}-5 \mathrm{I}_{4}{ }_{4}(0.00,0.32,0.63,0.95),-0.35,-0.03,+0.28,0.60,0.92,1.23,1.55$.

${ }^{5} \mathrm{G}_{4}-5 \mathrm{I}_{4}^{\prime}(0.55,1.10,1.65,2.20),-1.05,-0.50,+0.05,0.60,1.15,1.70,2.25,2.80$.

${ }^{5} \mathrm{G}_{4}-5 \mathrm{I}_{5}^{\prime}(0.00,0.25,0.50,0.75,1.00),-0.10,+0.15,0.40,0.65,0.90,1.15,1.40,1.65,1.90$.

${ }^{5} \mathrm{G}_{5}-\mathrm{I}^{\prime}{ }_{4}(0.00,0.67,1.33,2.00,2.67),-1.40,-0.73,-0.07,+0.60,1.27,1.93,2.60,3.27,3.93$.

${ }^{5} \mathrm{G}_{5}-5 \mathrm{I}^{\prime}{ }_{5}(0.37,0.73,1.10,1.47,1.83),-0.57,-0.20,+0.17,0.53,0.90,1.27,1.63,2.00,2.37,2.73$.

${ }^{8} \mathrm{G}_{5}{ }^{-5} \mathrm{I}^{\prime}{ }_{6}(0.00,0.20,0.39,0.59,0.78,0.98), 0.10,0.29,0.49,0.68,0.88,1.07,1.27,1.46,1.66,1.85,2.05$.

${ }^{8} \mathrm{G}_{6}-{ }^{5} \mathrm{I}_{5}{ }_{5}(0.09,0.43,0.87,1.30,1.73,2.17),-0.83,-0.40,+0.03,0.47,0.90,1.33,1.77,2.20,2.63,3.07,3.50$.

${ }^{3} \mathrm{G}_{6}-5 \mathrm{I}_{6}^{\prime}(0.26,0.52,0.79,1.05,1.31,1.5 \%),-0.24,+0.02,0.29,0.55,0.81,1.07,1.33,1.60,1.86,2.12,2.38,2.64$.

${ }^{8} \mathrm{G}_{6}{ }^{5} \mathrm{I}_{7}{ }_{7}(0.00,0.15,0.31,0.46,0.62,0.77,0.93), 0.25,0.40,0.56,0.71,0.87,1.02,1.18,1.33,1.49,1.64,1.80,1.95,2.11$.

${ }^{6} \mathrm{H}_{3}-5 \mathrm{H}^{\prime}{ }_{3}(0.00), 0.50$.

$\left.{ }^{5} \mathrm{H}_{3}{ }^{5}{ }^{-5} \mathrm{H}^{\prime}{ }^{-5} \mathrm{H}_{3}^{\prime}{ }_{3}\right\}(0.60,0.40,0.80,1.20),-0.30,+0.10,0.50,0.90,1.30,1.70,2.10$.

${ }^{5} \mathrm{H}_{4}-{ }^{5} \mathrm{H}_{4}^{\prime}(0.00), 0.90$.

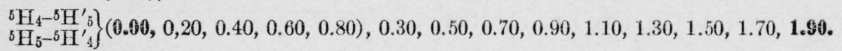

${ }^{5} \mathrm{H}_{5}{ }^{5} \mathrm{H}^{\prime}{ }_{5}(0.00), 1.10$.

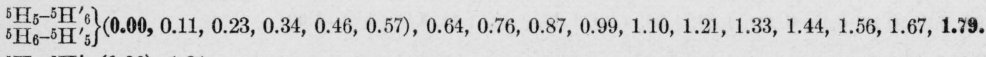

${ }^{8} \mathrm{H}_{6}-5 \mathrm{H}^{\prime}{ }_{6}(0.00), 1.21$.

$\left.{ }^{5} \mathrm{H}_{6}-{ }^{5} \mathrm{H}^{\prime} 7\right\}(0.00,0.07,0.14,0.21,0.29,0.36,0.43), 0.86,0.93,1.00,1.07,1.14,1.21,1.29,1.36,1.43,1.50,1.57,1.64$, $\left.{ }^{5} \mathrm{H}_{7}-{ }^{5} \mathrm{H}_{6}^{\prime}\right\}^{0}$ 1.71.

${ }^{8} \mathrm{H}_{7}-{ }^{5} \mathrm{H}_{7}^{\prime}(0.00), 1.29$.

${ }^{8} \mathrm{H}_{3}-5 \mathrm{I}_{4}(0.00,0.10,0.20,0.30), 0.30,0.40,0.50,0.60,0.70,0.80,0.90$.

${ }^{6} \mathrm{H}_{4}{ }^{-5} \mathrm{I}_{4}(0.30,0.60,0.90,1.20),-0.30,0.00,0.30,0.60,0.90,1.20,1.50,1.80$.

${ }^{5} \mathrm{H}_{4}-{ }^{5} \mathrm{I}_{5}(0.00), 0.90$.

${ }^{5} \mathrm{H}_{5}{ }^{-5} \mathrm{I}_{4}(0.00,0.50,1.00,1.50,2.00),-0.90,-0.40,+0.10,0.60,1.10,1.60,2.10,2.60,3.10$.

${ }^{6} \mathrm{H}_{5}{ }^{-5} \mathrm{I}_{5}(0.20,0.40,0.60,0.80,1.00), 0.10,0.30,0.50,0.70,0.90,1.10,1.30,1.50,1.70,1.90$.

${ }^{8} \mathrm{H}_{5}{ }^{-5} \mathrm{I}_{6}(0.00,0.03,0.06,0.09,0.11,0.14), 0.93,0.96,0.99,1.01,1.04,1.07,1.10,1.13,1.16,1.19,1.21$.

${ }^{5} \mathrm{H}_{6}{ }^{-5} \mathrm{I}_{5}(0.00,0.31,0.63,0.94,1.26,1.57),-0.36,-0.04,+0.27,0.59,0.90,1.21,1.53,1.86,2.16,2.47$, 2.79.

${ }^{8} \mathrm{H}_{6}-{ }^{5} \mathrm{I}_{6}(0.14,0.29,0.43,0.57,0.71,0.86), 0.36,0.50,0.64,0.79,0.93,1.07,1.21,1.36,1.50,1.64,1.79,1.93$.

${ }^{5} \mathrm{H}_{6}{ }^{-5} \mathrm{I}_{7}(0.00,0.04,0.07,0.11,0.14,0.18,0.21), 0.96,1.00,1.04,1.11,1.14,1.18,1.21,1.25,1.29,1.32,1.36,1.39$.

${ }^{5} \mathrm{H}_{7}-{ }^{-5} \mathrm{I}_{6}(0.00,0.21,0.43,0.64,0.86,1.07,1.29), 0.00,0.21,0.43,0.64,0.86,1.07,1.29,1.50,1.71,1.93,2.14,2.36$, 2.5\%.

${ }^{5} \mathrm{H}_{7}-{ }^{-5} \mathrm{I}_{7}(0.11,0.21,0.32,0.43,0.54,0.64,0.75), 0.54,0.64,0.75,0.86,0.96,1.07,1.18,1.29,1.39,1.50,1.61,1.71$, $1.82,1.93$.

${ }^{5} \mathrm{H}_{7}-{ }^{5} \mathrm{I}_{8}(0.00,0.04,0.07,0.11,0.14,0.18,0.21,0.25), 1.00,1.04,1.07,1.11,1.14,1.18,1.21,1.25,1.29,1.32,1.36$, $1.39,1.43,1.46,1.50$.

${ }^{5} \mathrm{I}_{4}-5 \mathrm{I}^{\prime}{ }_{4}(0.00), 0.60$.

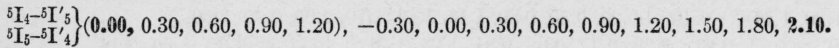

${ }^{6} \mathrm{I}_{5}-5 \mathrm{I}_{5}^{\prime}(0.00), 0.90$.

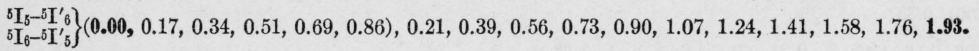

${ }^{5} \mathrm{I}_{6}-5 \mathrm{I}_{6}^{\prime}(0.00), 1.07$.

$\left.{ }^{5} \mathrm{I}_{\theta}-5 \mathrm{I}^{\prime}\right]_{7}(0.00,0.11,0.21,0.32,0.43,0.54,0.64), 0.54,0.64,0.75,0.86,0.96,1.07,1.18,1.29,1.39,1.50,1.61,1.71$, $\left.{ }^{5} \mathrm{I}_{7}-{ }^{-5} \mathrm{I}_{6}{ }_{6}\right\}$ 1.82.

${ }^{B} \mathrm{I}_{7}-5 \mathrm{I}^{\prime}{ }_{7}(0.00), 1.18$.

$\left.{ }^{5} \mathrm{I}_{7-5}{ }^{5} \mathrm{I}^{\prime} 8\right](0.00,0.07,0.14,0.21,0.36,0.43,0.50), 0.75,0.82,0.89,0.96,1.04,1.11,1.18,1.25,1.32,1.39,1.47,1.54$, $\left.{ }^{5} \mathrm{I}_{8}-5 \mathrm{I}_{7}\right\}^{3} 1.61,1.68,1.75$.

${ }^{5} \mathrm{I}_{8}-5 \mathrm{I}_{8}{ }_{8}(0.00), 1.25$. 
TABLE 10.-Theoretical Zeeman effects (sepiet syștem)

[Landé $g$ values]

\begin{tabular}{|c|c|c|c|c|c|c|c|c|c|c|c|c|c|c|c|c|c|c|c|c|}
\hline$l^{j}$ & 0 & 1 & 2 & 3 & 4 & 5 & 6 & 7 & 8 & 9 & 0 & 1 & 2 & 3 & 4 & 5 & 6 & 7 & 8 & 9 \\
\hline $\mathrm{s}$ & & & & $\frac{24}{12}$ & & & & & & & & & & 2. 000 & & & & & & \\
\hline$P$ & & & $\frac{14}{8}$ & $\frac{23}{12}$ & $\frac{38}{28}$ & & & & & & & & 2. 333 & 1. 917 & 1. 750 & & & & & \\
\hline$D$ & & $\frac{8}{2}$ & $\frac{12}{8}$ & $\frac{21}{12}$ & $\frac{33}{20}$ & $\frac{4}{3}{ }^{8}$ & & & & & & 3.000 & 2. 000 & 1. 750 & 1. 650 & 1.600 & & & & \\
\hline$F$ & $\div$ & $\frac{3}{3}$ & $\frac{9}{6}$ & $\frac{13}{1 \frac{3}{2}}$ & $\frac{30}{20}$ & $\frac{48}{30}$ & $\frac{33}{52}$ & & & & $\frac{0}{0}$ & 1. 500 & 1. 500 & 1. 500 & 1. 500 & 1.500 & 1. 500 & & & \\
\hline$G$ & & $-\frac{1}{2}$ & $\frac{5}{8}$ & $\frac{14}{13}$ & $\frac{28}{2} 0$ & $\frac{41}{30}$ & $\frac{39}{42}$ & $\frac{29}{53}$ & & & & -0.500 & 0.833 & 1.167 & 1. 300 & 1. 367 & 1.405 & 1.429 & & \\
\hline H & & & $\frac{0}{6}$ & $\frac{9}{12}$ & $\frac{21}{20}$ & $3 \frac{3}{6}$ & $\frac{34}{13}$ & $\frac{5}{5}$ & $\frac{99}{12}$ & & & & 0.000 & 0.750 & 1.050 & 1.200 & 1. 286 & 1. 339 & 1. 375 & \\
\hline I & & & & $\frac{3}{12}$ & $\frac{1}{2} 8$ & $\frac{30}{30}$ & $\frac{43}{4 \frac{3}{2}}$ & $\frac{8}{5}$ & $\frac{93}{72}$ & 130 & & & & 0.250 & 0.750 & 1.000 & 1. 143 & 1. 232 & 1. 292 & 1. 333 \\
\hline
\end{tabular}


TABLE 10.-Theoretical Zeeman effects (septet system)-Continued

${ }^{7} \mathrm{~S}_{3}-{ }^{7} \mathrm{~S}_{3}{ }_{3}(0.00), 2.00$.

${ }^{7} \mathrm{~S}_{3}-{ }^{7} \mathrm{P}_{2}(0.00,0.33,0.67), 1.33,1.67,2.00,2.33,2.67$.

${ }^{7} \mathrm{~S}_{3}{ }^{7} \mathrm{P}_{3}(0.08,0.17,0.25), 1.75,1.83,1.92,2.00,2.08,2.17$.

${ }^{7} \mathrm{~S}_{3}{ }^{7} \mathrm{P}_{4}(0.00,0.25,0.50,0.75), 1.00,1.25,1.50,1.75,2.00,2.25,2.50$.

${ }^{7} \mathrm{~S}_{3}-{ }^{7} \mathrm{D}_{2}{ }_{2}(0.00), 2.00$.

${ }_{7} \mathrm{~S}_{3}-{ }^{7} \mathrm{D}^{\prime}{ }_{3}(0.25,0.50,0.75), 1.25,1.50,1.75,2.60,2.25,2.50$.

${ }^{7} \mathrm{~S}_{3}-{ }^{7} \mathrm{D}_{4}^{\prime}(0.00,0.35,0.70,1.05), 0.60,0.95,1.30,1.65,2.00,2.35,2.70$.

${ }^{7} \mathrm{P}_{2}-7 \mathrm{P}^{\prime}{ }_{2}(0.00), 2.33$.

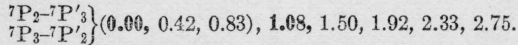

${ }^{7} \mathrm{P}_{3}-7 \mathrm{P}_{3}^{\prime}(0.00), 1.92$.

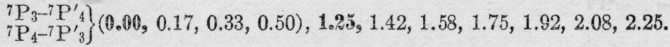

${ }^{7} \mathrm{P}_{4}-7 \mathrm{P}^{\prime}{ }_{4}(0.00), 1.75$

${ }^{7} \mathrm{P}_{2}-7 \mathrm{D}_{1}(0.00,0.67), \mathbf{1 . 6 7}, 2.33,3.00$.

${ }^{7} \mathrm{P}_{2}-7 \mathrm{D}_{2}(0.33,0.67), 1.67$, 2.60, 2.33, 2.67 .

${ }^{7} \mathrm{P}_{2}-7 \mathrm{D}_{3}(0.00,0.58,1.17), 0.58,1.17,1.75,2.33,2.92$.

${ }^{7} \mathrm{P}_{3}-{ }^{7} \mathrm{D}_{2}(0.00,0.08,0.17), 1.75,1.83,1.92,2.00,2.08$.

${ }^{7} \mathrm{P}_{3}{ }^{7} \mathrm{D}_{3}(0.17,0.33,0.50), 1.42,1.58,1.75,1.9 \%, 2.08,2.25$.

${ }^{7} \mathrm{P}_{3}-7 \mathrm{D}_{4}(0.00,0.27,0.53,0.80), 0.85,1.12,1.38,1.65,1.92,2.18,2.45$.

${ }^{7} \mathrm{P}_{4}-7 \mathrm{D}_{3}(0.00), 1.75$.

${ }^{7} \mathrm{P}_{4}{ }^{7} \mathrm{D}_{4}(0.10,0.20,0.30,0.40), 1.35,1.45,1.55,1.65,1.75,1.85,1.95,2.05$.

${ }^{7} \mathrm{P}_{4}{ }^{7} \mathrm{D}_{5}(0.00,0.15,0.30,0.45,0.60), 1.00,1.15,1.30,1.45,1.60,1.75,1.90,2.05,2.20$.

$7 \mathrm{P}_{2}{ }^{7} \mathrm{~F}^{\prime}{ }_{1}(0.00,0.83), 1.50,2.33,3.1 \%$.

$7 \mathrm{P}_{2}-7 \mathrm{~F}_{2}^{\prime}(0.83, \mathbf{1 . 6 7}), 0.67,1.57,2.33,3.17$.

${ }^{7} \mathrm{P}_{2}-7 \mathrm{~F}^{\prime}{ }_{3}(0.00,0.83,1.67),-0.17,+0.67,1.50,2.33,3.17$.

$7 \mathrm{P}_{3}-7 \mathrm{~F}_{2}{ }_{2}(0.00,0.42,0.83), 1.08,1.50,1.92,2.33,2.75$.

${ }^{7} \mathrm{P}_{3}-7 \mathrm{~F}^{\prime}{ }_{3}(0.42,0.83,1.25), 0.67,1.08,1.50,1.82,2.33,2.75$.

${ }^{7} \mathrm{P}_{3}-7 \mathrm{~F}^{\prime}{ }_{4}(0.00,0.42,0.83,1.25), 0.25,0.67,1.08,1.50,1.92,2.33,2.75$.

${ }^{7} \mathrm{P}_{4}{ }^{7}{ }^{7} \mathrm{~F}^{\prime}{ }_{3}(0.00,0.25,0.50,0.75), 1.00,1.25,1.50,1.75,2.00,2.25$, 2.50.

${ }^{7} \mathrm{P}_{4}{ }^{7} \mathrm{~F}^{\prime}{ }_{4}(0.25,0.50,0.75,1.00), 0.75,1.00,1.25,1.50,1.75,2.00,2.25,2.50$.

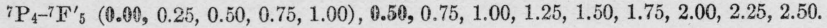

${ }^{7} \mathrm{D}_{1}{ }^{7} \mathrm{D}_{1}^{\prime}(0.00), 3.00$.

$\left.{ }^{7} \mathrm{D}_{1}{ }^{7} \mathrm{D}_{2}-\mathrm{D}^{\prime} \mathrm{D}^{\prime}{ }_{1}^{\prime}\right\}(0.00,1.00), \mathbf{1 . 0 0 ,} 2.00,3.00$.

${ }^{7} \mathrm{D}_{2}{ }^{7} \mathrm{D}_{2}^{\prime}(0.00), 2.00$.

$\left.{ }^{7} \mathrm{D}_{2}-7 \mathrm{D}^{\prime} \mathrm{D}_{3} \mathrm{D}^{7} \mathrm{D}_{2}\right\}(0.90,0.25,0.50), 1.25,1.50,1.75,2.00,2.25$.

${ }^{7} \mathrm{D}_{3}-7 \mathrm{D}_{3}^{\prime}(0.00), 1.75$.

$\left.{ }^{7} \mathrm{D}_{3^{-7}-{ }^{7} \mathrm{D}_{4}^{\prime}{ }^{\prime} \mathrm{D}_{3}^{\prime}} \mathrm{D}_{3}\right\}(0.00,0.10,0.20,0.30), 1.35,1.45,1.55,1.65,1.75,1.85,1.95$.

$7 \mathrm{D}_{4}{ }^{-7} \mathrm{D}_{4}(0.00), 1.65$

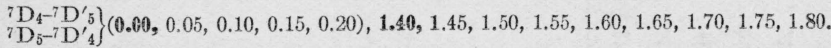

${ }^{7} \mathrm{D}_{5}^{-}{ }^{7} \mathrm{D}_{5}^{\prime}(0.00), 1.60$.

${ }^{7} \mathrm{D}_{1}{ }^{-7} \mathrm{~F}_{0}(0.00), 3.00$.

${ }^{7} \mathrm{D}_{1}{ }^{7} \mathrm{~F}_{1}(1.50), 1.50,3.00$.

${ }^{7} \mathrm{D}_{1}{ }^{7} \mathrm{~F}_{2}(0.00,1.50), 0.00,1.50,3.00$.

${ }^{7} \mathrm{D}_{2}{ }^{7} \mathrm{~F}_{1}(0.00,0.50), 1.50,2.00,2.30$.

$7 \mathrm{D}_{2}-7 \mathrm{~F}_{2}(0.50,1.00), 1.00,1.50,2.00,2.50$.

${ }^{7} \mathrm{D}_{2}{ }^{-7} \mathrm{~F}_{3}(0.00,0.50,1.00), 0.50,1.00,1.50,2.00,2.50$.

${ }^{7} \mathrm{D}_{3}-7 \mathrm{~F}_{2}(0.00,0.25,0.50), 1.25,1.50,1.75,2.00,2.25$.

${ }^{7} \mathrm{D}_{3}-7 \mathrm{~F}_{3}(0.25,0.50,0.75), 1.00,1.25,1.50,1.75,2.00,2.25$.

${ }^{7} \mathrm{D}_{3}{ }^{-7} \mathrm{~F}_{4}(\mathbf{0 . 0 0}, 0.25,0.50,0.75), 0.75,1.00,1.25,1.50,1.75,2.00,2.25$.

$7 \mathrm{D}_{4}-7 \mathrm{~F}_{3}(0.00,0.15,0.30,0.45), 1.20,1.35,1.50,1.65,1.80,1.95, \mathbf{2 . 1 0}$.

${ }^{7} \mathrm{D}_{4}{ }^{-7} \mathrm{~F}_{4}(0.15,0.30,0.45,0.60), 1.05,1.20,1.35,1.50,1.65,1.80,1.95,2.10$.

$7 \mathrm{D}_{4}-7 \mathrm{~F}_{5}(0.60,0.15,0.30,0.45,0.60), 0.90,1.05,1.20,1.35,1.50,1.65,1.80,1.95,2.10$.

${ }^{7} \mathrm{D}_{5}{ }^{-7} \mathrm{~F}_{4}(0.00,0.10,0.20,0.30,0.40), 1.20,1.30,1.40,1.50,1.60,1.70,1.80,1.90,2.00$.

${ }^{7} \mathrm{D}_{5}{ }^{-7} \mathrm{~F}_{5}(0.10,0.20,0.30,0.40,0.50), 1.10,1.20,1.30,1.40, \mathbf{1 . 5 0}, \mathbf{1 . 6 0}, 1.70,1.80,1.90,2.00$.

${ }^{7} \mathrm{D}_{5}{ }^{-7} \mathrm{~F}_{6}(0.00,0.10,0.20,0.30,0.40,0.50), 1.00,1.10,1.20,1.30,1.40,1.50,1.60,1.70,1.80,1.90,2.00$, 
TABLE 10.-Theoretical Zeeman effects (septet system)-Continued

${ }^{7} \mathrm{D}_{1}-{ }^{7} \mathrm{G}_{1}^{\prime}(3.50),-0.50,3.00$.

${ }^{7} \mathrm{D}_{1}{ }^{-7} \mathrm{G}^{\prime}{ }_{2}(0.00,2.17),-1.33,+0.83,3.00$.

${ }^{7} \mathrm{D}_{2}-{ }^{7} \mathrm{G}^{\prime}{ }_{1}(0.00,2.50), 0.50,2.00,4.50$.

${ }^{7} \mathrm{D}_{2}{ }^{-7} \mathrm{G}_{2}^{\prime}(1.17,2.33), 0.33,0.83,2.00,3.17$.

${ }^{7} \mathrm{D}_{2}{ }^{-7} \mathrm{G}_{3}{ }_{3}(0.00,0.83,1.67),-0.50,+0.33,1.17,2.00,2.83$.

${ }^{7} \mathrm{D}_{3}-{ }^{7} \mathrm{G}^{\prime}{ }_{2}(0.00,0.92,1.83), 0.08,0.83,1.75,2.67,3.58$.

${ }^{7} \mathrm{D}_{3}{ }^{7} \mathrm{G}^{\prime}{ }_{3}(0.58,1.17,1.75), 0.00,0.58,1.17,1.75,2.33,2.92$.

${ }^{7} \mathrm{D}_{3}{ }^{-7} \mathrm{G}_{4}^{\prime}(0.00,0.45,0.90,1.35), 0.05,0.40,0.85,1.30,1.75,2.20,2.65$.

${ }^{7} \mathrm{D}_{4^{-7}} \mathrm{G}^{\prime}{ }_{3}(0.00,0.48,0.97,1.45), 0.20,0.68,1.17,1.65,2.13,2.62,3.10$.

${ }^{7} \mathrm{D}_{4^{-7}} \mathrm{G}_{4}^{\prime}(0.35,0.70,1.05, \mathbf{1 . 4 0}), 0.25,0.60,0.95, \mathbf{1 . 3 0}, \mathbf{1 . 6 5}, 2.00,2.35,2.70$.

${ }^{7} \mathrm{D}_{4}{ }^{7} \mathrm{G}^{\prime}{ }_{5}(0.90,0.28,0.57,0.85,1.13), 0.23,0.52,0.80,1.08,1.37,1.65,1.93,2.22,2.50$.

${ }^{7} \mathrm{D}_{5}{ }^{7} \mathrm{G}^{\prime}{ }_{4}(0.00,0.30,0.60,0.90,1.20), 0.40,0.70,1.00,1.30,1.60,1.90,2.20,2.50,2.80$.

${ }^{7} \mathrm{D}_{5}{ }^{-7} \mathrm{G}^{\prime}{ }_{5}(0.23,0.47,0.70,0.93, \mathbf{1 . 1 7}), 0.43,0.67,0.90,1.13, \mathbf{1 . 3 7}, \mathbf{1 . 6 0}, 1.83,2.07,2.30,2.53$.

${ }^{7} \mathrm{D}_{5}{ }^{-7} \mathrm{G}_{6}^{\prime}(0.00,0.20,0.39,0.59,0.78,0.98), 0.43,0.62,0.82,1.01,1.21,1.40,1.60,1.80,1.99,2.19,2.38$.

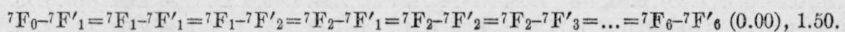

${ }^{7} \mathrm{~F}_{0}{ }^{-7} \mathrm{G}_{1}(0.00), 0.50$.

${ }^{7} \mathrm{~F}_{1}-{ }^{7} \mathrm{G}_{1}(2.00),-0.50,1.50$.

${ }^{7} \mathrm{~F}_{1}-{ }^{7} \mathrm{G}_{2}(0.00,0.67), 0.1 \%, 0.83,1.50$.

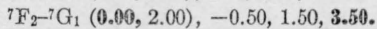

${ }^{7} \mathrm{~F}_{2}-7 \mathrm{G}_{2}(0.67,1.33), 0.17,0.83,1.50,2.17$.

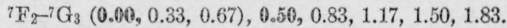

${ }^{7} \mathrm{~F}_{3}-{ }^{-7} \mathrm{G}_{2}(0.00,0.67,1.33), 0.17,0.83,1.50,2.17,2.83$.

${ }^{7} \mathrm{~F}_{3}-7 \mathrm{G}_{3}(0.33,0.67,1.00), 0.50,0.83, \mathbf{1 . 1 7}, \mathbf{1 . 5 0}, 1.83,2.17$.

${ }^{7} \mathrm{~F}_{3}-{ }^{7} \mathrm{G}_{4}(0.00,0.20,0.40,0.60), 0.70,0.90,1.10,1.30,1.50,1.70,1.90$.

${ }^{7} \mathrm{~F}_{4}-{ }^{7} \mathrm{G}_{3}(0.00,0.33,0.67,1.00), 0.50,0.83,1.17,1.50,1.83,2.17,2.50$.

${ }^{7} \mathrm{~F}_{4}{ }^{-7} \mathrm{G}_{4}(0.20,0.40,0.60,0.80), 0.70,0.90,1.10,1.30,1.59,1.70,1.90,2.10$.

${ }^{7} \mathrm{~F}_{4}{ }^{7} \mathrm{C}_{5}(0.00,0.13,0.27,0.40,0.53), 0.83,0.97,1.10,1.23,1.37,1.50,1.63,1.77,1.90$.

${ }^{7} \mathrm{~F}_{5}-{ }^{7} \mathrm{G}_{4}(0.60,0.20,0.40,0.60,0.80), 0.70,0.90,1.10,1.30,1.50,1.70,1.90, \mathbf{2 . 3 0}$.

${ }^{7} \mathrm{~F}_{5}{ }^{-7} \mathrm{G}_{5}(0.13,0.27,0.40,0.53,0.67), 0.83,0.97,1.10,1.23,1.37,1.50,1.63,1.77,1.90,2.03$.

${ }^{7} \mathrm{~F}_{5}-{ }^{7} \mathrm{G}_{6}(0.00,0.10,0.19,0.29,0.38,0.48), 0.93,1.02,1.12,1.22,1.31,1.41,1.50,1.60,1.69,1.79,1.88$.

${ }^{7} \mathrm{~F}_{6}{ }^{7} \mathrm{G}_{5}(\mathbf{0 . 0 0}, 0.13,0.27,0.40,0.53,0.67), 0.83,0.97,1.10,1.23,1.37,1.50,1.63,1.77,1.90,2.03,2.17$.

${ }^{7} \mathrm{~F}_{0^{-}}{ }^{7} \mathrm{G}_{0}(0.10,0.19,0.29,0.38,0.48,0.57), 0.93,1.02,1.12,1.22,1.31,1.41,1.50,1.60,1.69,1.79,1.88,1.98$.

${ }^{7} \mathrm{~F}_{6}{ }^{-7} \mathrm{G}_{7}(\mathbf{0 . 0 0}, 0.07,0.14,0.21,0.29,0.36,0.43), 1.00,1.07,1.14,1.21,1.29,1.36,1.43,1.50,1.57,1.64,1.71,1.78,1.86$.

${ }^{7} \mathrm{~F}_{1}-{ }^{7} \mathrm{H}_{2}^{\prime}(0.00,1.50), 0.00,1.50$.

${ }^{7} \mathrm{~F}_{2}-{ }^{7} \mathrm{H}^{\prime}{ }_{2}(1.50,3.00), 0.00,1.50,3.00$.

${ }^{7} \mathrm{~F}_{2}-7 \mathrm{H}^{\prime}{ }_{3}(0.00,0.75,1.50),-0.75,0.00,0.75,1.50,2.25$.

${ }^{7} \mathrm{~F}_{3}-7 \mathrm{H}_{2}{ }_{2}(0.00,1.50,3.00),-1.50,0.00,1.50,3.00,4.50$.

${ }^{7} \mathrm{~F}_{3}-7 \mathrm{H}^{\prime}{ }_{3}(0.75,1.50,2.25),-0.75,0.00,0.75,1.50,2.25,3.00$.

${ }^{7} \mathrm{~F}_{3}-7 \mathrm{H}^{\prime}{ }_{4}(0.00,0.45,0.90,1.35),-0.30,+0.15,0.60,1.05,1.50,1.95,2.40$.

${ }^{7} \mathrm{~F}_{4^{-}-{ }^{7} \mathrm{H}^{\prime}{ }_{3}(0.00,0.75,1.50,2.25)}-0.75,0.00,0.75,1.50,2.25,3.00,3.75$.

${ }^{7} \mathrm{~F}_{4}{ }^{7} \mathrm{H}_{4}^{\prime}(0.45,0.90,1.35,1.80),-0.30,+0.15,0.60, \mathbf{1 . 0 5}, \mathbf{1 . 5 0}, 1.95,2.40,2.85$.

${ }^{7} \mathrm{~F}_{4}{ }^{-7} \mathrm{H}_{5}{ }_{5}(0.00,0.30,0.60,0.90,1.20), 0.00,0.30,0.60,0.90,1.20,1.50,1.80,2.10,2.40$.

${ }^{7} \mathrm{~F}_{5}{ }^{-7} \mathrm{H}_{4}^{\prime}(0.00,0.45,0.90,1.35,1.80),-0.30,+0.15,0.60,1.05,1.50,1.95,2.40,1.85,3.30$.

${ }^{7} \mathrm{~F}_{5}{ }^{7} \mathrm{H}^{\prime}{ }_{5}(0.30,0.60,0.90,1.20, \mathbf{1 . 5 0}), 0.00,0.30,0.60,0.90,1.20,1.50,1.80,2.10,2.40,2.70$.

${ }^{7} \mathrm{~F}_{5}-{ }^{7} \mathrm{H}_{6}{ }_{6}(0.00,0.21,0.43,0.64,0.86,1.07), 0.21,0.43,0.64,0.86,1.07,1.29,1.50,1.71,1.93,2.14,2.36$.

${ }^{7} \mathrm{~F}_{6}{ }^{-7} \mathrm{H}^{\prime}{ }_{5}(\mathrm{0.00}, 0.30,0.60,0.90,1.20,1.50), 0.00,0.30,0.60,0.90,1.20,1.50,1.80,2.10,2.40,2.70,3.00$.

${ }^{7} \mathrm{~F}_{6}{ }^{7} \mathrm{H}^{\prime}{ }_{6}(0.21,0.43,0.64,0.86,1.07,1.28), 0.21,0.43,0.64,0.86,1.07,1.28,1.50,1.71,1.93,2.14,2.36,2.57$.

${ }^{7} \mathrm{~F}_{6}{ }^{-7} \mathrm{H}^{\prime}{ }_{7}(0.00,0.16,0.32,0.48,0.64,0.80,0.96), \mathbf{0 . 3 7}, 0.54,0.70,0.86,1.02,1.18,1.34,1.50,1.66,1.82,1.98,2.14,2.30$.

${ }^{7} \mathrm{G}_{1}-7 \mathrm{G}_{1}^{\prime}(0.00), 0.50$

$\left.\begin{array}{l}{ }^{7} \mathrm{G}_{1}-7 \mathrm{G}^{\prime}{ }_{2} \\ { }^{7} \mathrm{G}_{2}{ }^{7} \mathrm{G}^{\prime}{ }_{1}\end{array}\right\}(0.00,1.33),-0.50,+0.83,2.17$.

${ }^{7} \mathrm{G}_{2}-7 \mathrm{G}_{2}^{\prime}(0.00), 0.83$.

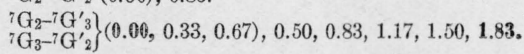

${ }^{7} \mathrm{G}_{3}-7 \mathrm{G}^{\prime}{ }_{3}(0.00), 1.17$.

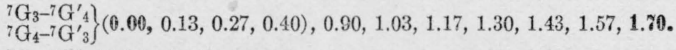

${ }^{7} \mathrm{G}_{4^{-7}} \mathrm{G}^{\prime}{ }_{4}(0.00), 1.30$

$\left.{ }^{7} \mathrm{G}_{4}{ }^{7} \mathrm{G}_{5}^{-7} \mathrm{G}^{-7} \mathrm{G}_{5}^{\prime}{ }_{4}^{\prime}\right\}(0.00,0.07,0.13,0.20,0.27), 1.10,1.17,1.23,1.30,1.43,1.50,1.57,1.63$.

${ }^{7} \mathrm{G}_{5}{ }^{-7} \mathrm{G}^{\prime}{ }_{5}(0.00), 1.37$.

$\left.{ }^{7} \mathrm{G}_{5^{-7}}^{7} \mathrm{G}^{7}{ }_{6}{ }_{6}^{-7} \mathrm{G}_{5}{ }_{5}\right\}(0.00,0.04,0.08,0.11,0.15,0.19), 1.21,1.25,1.29,1.33,1.37,1.40,1.44,1.48,1.52,1.56,1.60$.

${ }^{7} \mathrm{G}_{6}-7 \mathrm{G}^{\prime}{ }_{6}(0.00), 1.40$.

$\left.{ }^{7} \mathrm{G}_{6-7} \mathrm{G}^{\prime} 7{ }^{(0.00,0.02,0.05}, 0.07,0.10,0.12,0.14\right), 1.29,1.31,1.33,1.36,1.38,1.40,1.43,1.45,1.48,1.50,1.52,1.55$, ${ }^{7} \mathrm{G}_{7}-{ }^{7} \mathrm{G}^{\prime}{ }_{6} \mathrm{H}^{1.5 \%}$.

${ }^{7} \mathrm{G}_{7}-{ }^{7} \mathrm{G}^{\prime}{ }_{7}(0.00), 1.43$. 
TABLE 10.-Theoretical Zeeman effects (septet system)-Continued

${ }^{7} \mathrm{G}_{1}{ }^{7} \mathrm{H}_{2}(0.00,0.50),-0.50,0.00,0.50$.

${ }^{7} \mathrm{G}_{2}-{ }^{7} \mathrm{H}_{2}(0.83, \mathbf{1 . 6 7}),-0.83,0.00,0.83,1.67$.

${ }^{7} \mathrm{G}_{2}{ }^{-7} \mathrm{H}_{3}(0.00,0.08,0.17), 0.58,0.67,0.75,0.83,0.92$.

${ }^{7} \mathrm{G}_{3}{ }^{7} \mathrm{H}_{2}(\boldsymbol{0 . 0 0}, 1.17,2.33),-1.17,0.00,1.17,2.33,3.50$.

${ }^{7} \mathrm{G}_{3}{ }^{7} \mathrm{H}_{3}(0.42,0.83,1.25), 0.08,0.33,0.75,1.17,1.58,2.00$.

${ }^{7} \mathrm{G}_{3}{ }^{-7} \mathrm{H}_{4}(0.00,0.12,0.23,0.35), 0.70,0.82,0.93,1.05,1.17,1.28,1.40$.

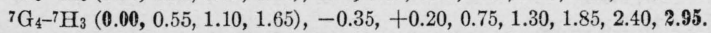

${ }^{7} \mathrm{G}_{4}{ }^{-7} \mathrm{H}_{4}(0.25,0.50,0.75,1.00), 0.30,0.55,0.80,1.05,1.30,1.55,1.80,2.05$.

${ }^{7} \mathrm{G}_{4}{ }^{-7} \mathrm{H}_{5}(\boldsymbol{\theta} .00,0.10,0.20,0.30,0.40), 0.80,0.90,1.00,1.10,1.20,1.30,1.40,1.50,1.60$.

${ }^{7} \mathrm{G}_{5}-{ }^{-7} \mathrm{H}_{4}(0.00,0.32,0.63,0.95,1.27), 0.10,0.42,0.73,1.05,1.37,1.68,2.00,2.32,2.63$.

${ }^{7} \mathrm{G}_{5}{ }^{7} \mathrm{H}_{5}(0.17,0.33,0.50,0.67,0.83), 0.53,0.70,0.87,1.03,1.20,1.37,1.53,1.70,1.87,2.03$.

${ }^{7} \mathrm{G}_{5}{ }^{-}{ }^{H_{6}}(\boldsymbol{\theta} .00,0.08,0.16,0.24,0.32,0.40), 0.88,0.96,1.04,1.12,1.20,1.29,1.37,1.45,1.53,1.61,1.69$.

${ }^{\top} \mathrm{G}_{6}{ }^{-7} \mathrm{H}_{5}(0.00,0.20,0.41,0.61,0.82,1.02), 0.38,0.59,0.79,1.00,1.20,1.40,1.61,1.81,2.02,2.22,2.43$.

${ }^{1} \mathrm{G}_{6}{ }^{7} \mathrm{H}_{6}(0.12,0.24,0.36,0.48,0.60, \mathbf{0 . 7 1}), 0.69,0.81,0.93,1.05,1.17, \mathbf{1 . 2 9}, 1.41,1.52,1.64,1.76,1.88,2.00$.

${ }^{7} \mathrm{G}_{6}{ }^{7} \mathrm{H}_{7}(\mathbf{0 . 0 0}, 0.07,0.13,0.20,0.26,0.33,0.39), 0.95,1.01,1.08,1.14,1.21,1.27,1.34,1.40,1.47,1.54,1.60,1.67,1.73$.

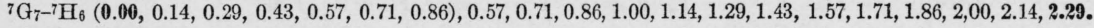

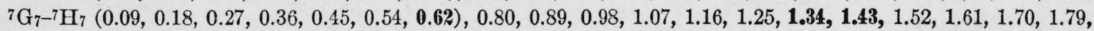
$1.88,1.96$.

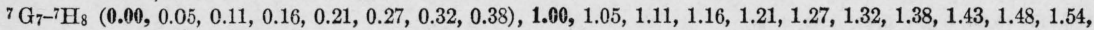
$1.59,1.64,1.70,1.75$.

${ }^{7} \mathrm{H}_{2}-{ }^{-} \mathrm{H}_{2}{ }_{2}(0.00), 0.00$ unaffected.

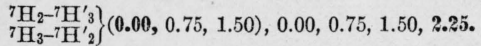

${ }^{7} \mathrm{H}_{3}-{ }^{7} \mathrm{H}_{3}^{\prime}(0.00), 0.75$.

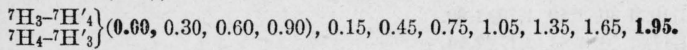

${ }^{7} \mathrm{H}_{4-7}{ }^{7} \mathrm{H}^{\prime}{ }_{4}(0.00), 1.05$.

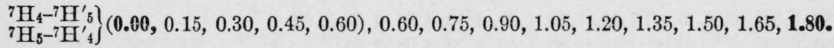

${ }^{7} \mathrm{H}_{5}{ }^{7} \mathrm{H}^{\prime}{ }_{5}(0.00), 1.20$.

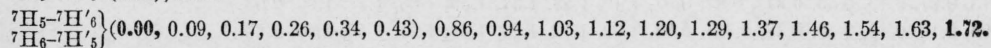

${ }^{7} \mathrm{H}_{6}-{ }^{7} \mathrm{H}_{6}^{\prime}(0.00), 1.29$.

${ }^{7} \mathrm{H}_{6}{ }^{-7} \mathrm{H}_{7}{ }^{7}(0.00,0.05,0.11,0.16,0.21,0.27,0.32), 1.02,1.07,1.13,1.18,1.23,1.29,1.34,1.39,1.45,1.50,1.56,1.61$, ${ }^{7} \mathrm{H}_{7}{ }^{-7} \mathrm{H}_{6}^{\prime}{ }^{\prime}{ }^{2} \mathbf{1 . 6 6}$.

${ }^{7} \mathrm{H}_{7}{ }^{7} \mathrm{H}^{\prime}{ }_{7}(0.00), 1.34$.

${ }^{7} \mathrm{H}_{7}{ }^{7} \mathrm{H}^{\prime} 8{ }^{\prime}(0.00,0.04,0.07,0.11,0.14,0.18,0.21,0.25), 1.13,1.16,1.20,1.23,1.27,1.30,1.34,1.38,1.41,1.45,1.48$, $\left.{ }^{7} \mathrm{H}_{8}{ }^{7} \mathrm{H}^{\prime}{ }_{7}\right\}^{7} 1.52,1.55,1.59,1.62$.

${ }^{7} \mathrm{H}_{8}{ }^{7} \mathrm{H}_{8}^{\prime}(0.00), 1.37$.

TAвLE 11.-Theoretical Zeeman effects (singlet-triplet intersysiem)

${ }^{1} \mathrm{~S}_{0^{-}-{ }^{-3} \mathrm{~S}_{1}}(0.00), 2.00$.

${ }^{1} \mathrm{~S}_{0}{ }^{-3} \mathrm{P}_{1}(0.00), 1.50$.

${ }^{1} \mathrm{~S}_{0^{-3}} \mathrm{D}^{\prime}{ }_{1}(0.00), 0.50$.

${ }^{1} \mathrm{P}_{1}{ }^{-3} \mathrm{~S}_{1}$ (1.00), 1.00, 2.00 .

${ }^{1} \mathrm{P}_{1}-\mathrm{P}^{-} \mathrm{P}_{0}(0.00), 1.00$.

${ }^{1} \mathrm{P}_{1}{ }^{-3} \mathrm{P}^{\prime}{ }_{1}(0.50), 1.00,1.50$.

${ }^{1} \mathrm{P}_{1}-3 \mathrm{P}_{2}^{\prime}(0.00,0.50), 1.00,1.50,2.00$.

${ }^{1} \mathrm{P}_{1} \_\mathrm{D}_{1}(0.50), 0.50,1.00$.

${ }^{1} \mathrm{P}_{1}-{ }^{-3} \mathrm{D}_{2}(0.00,0.17), 1.00,1.17,1.33$.

${ }^{1} \mathrm{P}_{1}{ }^{-3} \mathrm{~F}_{3}{ }_{3}(0.00,0.33), 0.33,0.67,1.00$.

${ }^{1} \mathrm{D}_{2}{ }^{-3} \mathrm{~S}_{1}^{\prime}(0.00,1.00), 0.00,1.00,2.00$.

${ }^{1} \mathrm{D}_{2}{ }^{3} \mathrm{P}_{1}(0.00,0.50), 0.50,1.00,1.50$.

${ }^{1} \mathrm{D}_{2}{ }^{-3} \mathrm{P}_{2}(0.50,1.00), 0.50,1.00,1.50,2.00$.

${ }^{1} \mathrm{D}_{2}{ }^{-3} \mathrm{D}_{1}^{\prime}(0.00,0.50), 0.50,1.00,1.50$.

${ }^{1} \mathrm{D}_{2}-{ }^{3} \mathrm{D}_{2}^{\prime}{ }_{2}(0.17,0.33), 0.83,1.00,1.17,1.33$.

${ }^{1} \mathrm{D}_{2}-{ }^{-3} \mathrm{D}_{3}^{\prime}(\mathbf{0 . 0 0}, 0.33,0.67), 0.67,1.00,1.33,1.67,2.00$.

${ }^{1} \mathrm{D}_{2}{ }^{-3} \mathrm{~F}_{2}(0.33,0.67), 0.33,0.67,1.00,1.33$.

${ }^{1} \mathrm{D}_{2}-{ }^{3} \mathrm{~F}_{3}(0.00,0.08,0.17), 0.92,1.00,1.08,1.17,1.25$. 
TABLE 11.-Theoretical Zeeman effects (singlet-triplet intersystem)-Continued

${ }^{1} \mathrm{D}_{2^{-3}} \mathrm{G}^{\prime}{ }_{3}(0.00,0.25,0.50), 0.25,0.50,0.75,1.00,1.25$.

${ }^{1} \mathrm{~F}_{3}-{ }^{-3} \mathrm{P}_{2}^{\prime}(0.00,0.50,1.00), 0.00,0.50,1.00,1.50,2.00$.

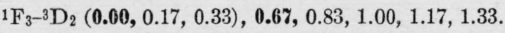

${ }^{1} \mathrm{~F}_{3}-{ }^{-3} \mathrm{D}_{3}(0.33,0.67,1.00), 0.33,0.67,1.00,1.33,1.67,2.00$.

${ }^{1} \mathrm{~F}_{3}-3 \mathrm{~F}^{\prime}{ }_{2}(0.00,0.33,0.67), 0.33,0.67,1.00,1.33,1.67$.

${ }^{1} \mathrm{~F}_{3}-{ }^{-3} \mathrm{~F}^{\prime}{ }_{3}(0.08,0.17,0.25), 0.83,0.92,1.00,1.08,1.17,1.25$.

${ }^{1} \mathrm{~F}_{3}{ }^{-3} \mathrm{~F}_{4}^{\prime}(0.00,0.25,0.50,0.75), 0.50,0.75,1.00,1.25,1.50,1.75$, 2.00.

${ }_{1} \mathrm{~F}_{3}-{ }^{-3} \mathrm{G}_{3}(0.25,0.50,0.75), 0.25,0.50,0.75,1.00,1.25,1.50$.

${ }^{1} \mathrm{~F}_{3}-{ }^{3} \mathrm{G}_{4}(0.00,0.05,0.10,0.15), 0.90,0.95,1.00,1.05,1.10,1.15,1.20$.

${ }^{1} \mathrm{~F}_{3}-{ }^{3} \mathrm{H}^{\prime}{ }_{4}(\mathbf{0 . 0 0}, 0.20,0.40,0.60), \mathbf{0 . 2 0}, 0.40,0.60,0.80,1.00,1.20,1.40$.

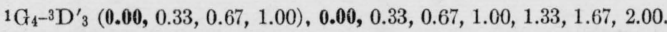

${ }^{1} \mathrm{G}_{4^{-3}} \mathrm{~F}_{3}(\mathbf{0 . 6 0}, 0.08,0.17,0.25), \mathbf{0 . 7 5}, 0.83,0.92,1.00,1.08,1.17,1.25$.

${ }^{1} \mathrm{G}_{4}{ }^{3} \mathrm{~F}_{4}(0.25,0.50,0.75,1.00), 0.25,0.50,0.75,1.00,1.25,1.50,1.75,2.00$.

${ }^{1} \mathrm{G}_{4^{-3}} \mathrm{G}^{\prime}{ }_{3}(0.00,0.25,0.50,0.75), 0.25,0.50,0.75,1.00,1.25,1.50,1.75$.

${ }^{1} \mathrm{G}_{4}{ }^{-3} \mathrm{G}^{\prime}{ }_{4}(0.05,0.10,0.15,0.20), 0.85,0.90,1.00,1.05,1.10,1.15,1.20$.

${ }^{1} \mathrm{G}_{4^{-3}} \mathrm{G}^{\prime}{ }_{5}(0.00,0.20,0.40,0.60,0.80), 0.40,0.60,0.80,1.00,1.20,1.40,1.60,1.80$, 2.00.

${ }^{1} \mathrm{G}_{4}{ }^{3} \mathrm{H}_{4}(0.20,0.40,0.60,0.80), 0.20,0.40,0.60,0.80,1.00,1.20,1.40,1.60$.

${ }^{1} \mathrm{G}_{4^{-3}} \mathrm{H}_{5}(0.00,0.03,0.07,0.10,0.13), 0.90,0.93,0.97,1.00,1.03,1.07,1.10,1.13,1.17$.

${ }^{1} \mathrm{G}_{4-3} \mathrm{I}_{5}{ }_{5}(0.00,0.17,0.33,0.50,0.67), 0.17,0.33,0.50,0.67,0.83,1.00,1.17,1.33,1.50$.

${ }^{1} \mathrm{H}_{5}{ }^{-3} \mathrm{~F}_{4}^{\prime}(0.00,0.25,0.50,0.75,1.00), 0.00,0.25,0.50,0.75,1.00,1.25,1.50,1.75,2.00$.

${ }^{1} \mathrm{H}_{5^{-3}} \mathrm{G}_{4}(\mathbf{0 . 6 0}, 0.05,0.10,0.15,0.20), \mathbf{0 . 8 0}, 0.85,0.90,0.95,1.00,1.05,1.10,1.15,1.20$.

${ }^{1} \mathrm{H}_{5}-{ }^{3} \mathrm{G}_{5}(0.20,0.40,0.60,0.80,1.00), 0.20,0.40,0.60,0.80,1.00,1.20,1.40,1.60,1.80,2.00$.

${ }^{1} \mathrm{H}_{5}{ }^{-3} \mathrm{H}_{4}^{\prime}(\mathbf{0 . 0 0}, 0.20,0.40,0.60,0.80), 0.20,0.40,0.60,0.80,1.00,1.20,1.40,1.60,1.80$.

${ }^{1} \mathrm{H}_{5}{ }^{-3} \mathrm{H}_{5}{ }_{5}(0.03,0.07,0.10,0.13,0.17), 0.87,0.90,0.93,0.97,1.00,1.03,1.07,1.10,1.13,1.17$.

${ }^{1} \mathrm{H}_{5}{ }^{-3} \mathrm{H}_{6}^{\prime}(0.00,0.17,0.33,0.50,0.67,0.83), 0.33,0.50,0.67,0.83,1.00,1.17,1.33,1.50,1.67,1.83,2.00$.

${ }^{1} \mathrm{H}_{5^{-3}} \mathrm{I}_{5}(0.17,0.33,0.50,0.67,0.83), 0.17,0.33,0.50,0.67,0.83,1.00,1.17,1.33,1.50,1.67$.

${ }^{1} \mathrm{H}_{5}{ }^{-3} \mathrm{I}_{6}(\mathbf{0 . 0 0}, 0.02,0.05,0.07,0.10,0.12), 0.90,0.93,0.95,0.98,1.00,1.02,1.05,1.07,1.10,1.12,1.14$.

${ }^{1} \mathrm{I}_{0^{-}}{ }^{-3} \mathrm{G}_{5}{ }_{5}(\mathbf{0 . 0 0}, 0.20,0.40,0.60,0.80,1.00), 0.00,0.20,0.40,0.60,0.80,1.00,1.20,1.40,1.60,1.80,2.00$.

${ }^{1} \mathrm{I}_{6}{ }^{-3} \mathrm{H}_{5}(\mathbf{0 . 0 0}, 0.03,0.07,0.10,0.13,0.17), \mathbf{0 . 8 3}, 0.87,0.90,0.93,0.97,1.00,1.03,1.07,1.10,1.13,1.17$.

${ }^{1} \mathrm{I}_{6}{ }^{-3} \mathrm{H}_{6}(0.17,0.33,0.50,0.67,0.83,1.00), 0.17,0.33,0.50,0.67,0.83, \mathbf{1 . 0 0}, 1.17,1.33,1.50,1.67,1.83,2.00$.

${ }^{1} \mathrm{I}_{6}-{ }^{-3} \mathrm{I}_{5}{ }_{5}(0.00,0.17,0.33,0.50,0.67,0.83), 0.17,0.33,0.50,0.67,0.83,1.00,1.17,1.33,1.50,1.67,1.83$.

${ }^{1} \mathrm{I}_{6}-{ }^{3} \mathrm{I}_{6}{ }_{6}(0.02,0.05,0.07,0.10,0.12, \mathbf{0 . 1 4}), 0.88,0.90,0.93,0.95,0.98,1.00,1.02,1.05,1.07,1.10,1.12,1.14$.

${ }^{1} \mathrm{I}_{6}-{ }^{-3} \mathrm{I}_{7}{ }_{7}(\mathbf{0 . 0 0}, 0.14,0.29,0.43,0.57,0.71,0.86), 0.29,0.43,0.57,0.71,0.86,1.00,1.14,1.29,1.43,1.57,1.71,1.86,2.00$.

TABLE 12.-Theoretical Zeeman effects (triplet-quintet intersystem)

${ }^{3} \mathrm{~S}_{1}-\mathrm{S}^{\prime} \mathrm{S}_{2}(0.00), 2.00$.

${ }^{8} \mathrm{~S}_{1}-5 \mathrm{P}_{1}(0.50), 2.00,2.50$.

${ }^{3} \mathrm{~S}_{1}-5 \mathrm{P}_{2}(0.00,0.17), 1.67,1.83,2.00$.

${ }^{3} \mathrm{~S}_{1}-5 \mathrm{D}^{\prime}{ }_{0}(0.00), 2.00$.

${ }^{3} \mathrm{~S}_{1}-5 \mathrm{D}^{\prime}{ }_{1}(0.50), 1.50,2.00$.

${ }^{3} \mathrm{~S}_{1}-5 \mathrm{D}_{2}^{\prime}(0.00,0.50), \mathbf{1 . 0 0}, 1.50,2.00$.

${ }^{3} \mathrm{P}_{1}{ }^{-5} \mathrm{~S}_{2}(0.00,0.50), 1.50,2.00,2.50$.

${ }^{3} \mathrm{P}_{2}-5 \mathrm{~S}_{2}(0.50,1.00), 1.00,1.50,2.00,2.50$.

${ }^{3} \mathrm{P}_{0}-5 \mathrm{P}^{\prime}{ }_{1}(0.00), 2.50$.

${ }^{3} \mathrm{P}_{1}-5 \mathrm{P}_{1}^{\prime}(1.00), 1.50,2.50$.

${ }^{3} \mathrm{P}_{1}-\mathrm{P}^{\prime}{ }_{2}(0.00,0.33), 1.50,1.83,2.17$.

${ }^{3} \mathrm{P}_{2}-{ }^{-5} \mathrm{P}_{1}^{\prime}(0.60,1.00), 0.50,1.50,2.50$.

${ }^{3} \mathrm{P}_{2}-5 \mathrm{P}_{2}^{\prime}(0.33,0.67), 1.17,1.50,1.83,2.17$.

${ }^{3} \mathrm{P}_{2}-5 \mathrm{P}_{3}^{\prime}(\mathbf{0 . 0 0}, 0.17,0.33), 1.33,1.50,1.67,1.83, \mathbf{2 . 0 0}$.

${ }^{3} \mathrm{P}_{0}-5 \mathrm{D}_{1}={ }^{3} \mathrm{P}_{1}-{ }^{-} \mathrm{D}_{1}={ }^{3} \mathrm{P}_{1}-6 \mathrm{D}_{2}={ }^{3} \mathrm{P}_{2}-5 \mathrm{D}_{1}=\ldots={ }^{3} \mathrm{P}_{2}-5 \mathrm{D}_{3}(0.00), 1.50$. 
TABLE 12.-Theoretical Zeeman effects (triplet-quintet intersystem)-Continued.

${ }^{3} \mathrm{P}_{0}-5 \mathrm{~F}^{\prime}{ }_{1}(0.00), 0.00$, unaffected.

${ }^{3} \mathrm{P}_{1}-5 \mathrm{~F}^{\prime}{ }_{1}(1.50), 0.00,1.50$.

${ }^{3} \mathrm{P}_{1}-5 \mathrm{~F}^{\prime}{ }_{2}(0.00,0.50), 0.50,1.00,1.50$.

${ }^{3} \mathrm{P}_{2}-5 \mathrm{~F}^{\prime}{ }_{1}(\mathbf{0 . 0 0}, 1.50), 0.00,1.50,3.00$.

${ }^{3} \mathrm{P}_{2}-5 \mathrm{~F}^{\prime}{ }_{2}(0.50,1.00), 0.50,1.00,1.50,2.00$.

${ }^{3} \mathrm{P}_{2}-5 \mathrm{~F}^{\prime}{ }_{3}(0.00,0.25,0.50), 0.75,1.00,1.25,1.50,1.75$.

${ }^{3} \mathrm{D}_{1}-5 \mathrm{~S}_{2}{ }_{2}(0.00,1.50), 0.50,2.00$, 3.50 0 .

${ }^{3} \mathrm{D}_{2}-{ }^{-5} \mathrm{~S}_{2}{ }_{2}(0.83, \mathbf{1 . 6 7}), 0.33, \mathbf{1 . 1 7}, \mathbf{2 . 0 0}, 2.83$.

${ }^{3} \mathrm{D}_{3}{ }^{-5} \mathrm{~S}_{2}{ }_{2}(0.00,0.67,1.33), 0.00,0.67,1.33,2.00,2.67$.

${ }^{3} \mathrm{D}_{1}{ }^{-5} \mathrm{P}_{1}(2.00), 0.50,2.50$.

${ }^{3} \mathrm{D}_{1}{ }^{-5} \mathrm{P}_{2}(0.00,1.33), 0.50,1.83,3.1 \%$.

${ }^{3} \mathrm{D}_{2}-5 \mathrm{P}_{1}(0.00,1.33),-0.17,+1.17,2.50$.

${ }^{3} \mathrm{D}_{2}{ }^{-5} \mathrm{P}_{2}(0.67,1.33), 0.50,1.17,1.83,2.50$.

${ }^{3} \mathrm{D}_{2}-5 \mathrm{P}_{3}(0.00,0.50,1.00), 0.67,1.17,1.67,2.17,2.6 \%$.

${ }^{3} \mathrm{D}_{3}-{ }^{5} \mathrm{P}_{2}(0.00,0.50,1.00), 0.33,0.83,1.33,1.83,2.33$.

${ }^{3} \mathrm{D}_{3}-{ }^{-5} \mathrm{P}_{3}(0.33,0.67 .1 .00), 0.67,1.00,1.33,1.67,2.00,2.33$.

${ }^{3} \mathrm{D}_{1}{ }^{5} \mathrm{D}_{0}^{\prime}(0.00), 0.50$.

${ }^{3} \mathrm{D}_{1}{ }^{-5} \mathrm{D}_{1}^{\prime}(1.00), 0.50,1.50$.

${ }^{3} \mathrm{D}_{1}{ }^{-5} \mathrm{D}_{2}^{\prime}(0.00,1.00), 0.50,1.50,2.50$.

${ }^{3} \mathrm{D}_{2}-5 \mathrm{D}_{1}^{\prime}(0.00,0.33), 0.83,1.17,1.50$.

${ }^{3} \mathrm{D}_{2}-5 \mathrm{D}_{2}^{\prime}{ }_{2}(0.33,0.67), 0.83,1.17,1.50,1.83$.

${ }^{3} \mathrm{D}_{2}{ }^{-5} \mathrm{D}_{3}^{\prime}{ }_{3}(0.00,0.33,0.67), 0.83,1.17,1.50,1.83, \mathbf{2 . 1 7}$.

${ }^{3} \mathrm{D}_{3}-{ }^{-5} \mathrm{D}_{2}^{\prime}(0.00,0.17,0.33), 1.00,1.17,1.33,1.50,1.67$.

${ }^{3} \mathrm{D}_{3}-5 \mathrm{D}_{3}{ }_{3}(0.17,0.33,0.50), 1.00,1.17,1.33,1.56,1.67,1.83$.

${ }^{3} \mathrm{D}_{3}-5 \mathrm{D}_{4}^{\prime}(0.00,0.17,0.33,0.50), 1.00,1.17,1.33,1.50,1.67,1.83,2.00$.

${ }^{3} \mathrm{D}_{1}{ }^{-5} \mathrm{~F}_{1}(0.50), 0.00,0.50$.

$3 \mathrm{D}^{-6} \mathrm{~F}_{2}(0.00,0.50), 0.50,1.00,1.50$.

${ }^{3} \mathrm{D}_{2}-5 \mathrm{~F}_{1}(0.00,1.17), 0.00,1.17,2.33$.

${ }^{3} \mathrm{D}_{2}-{ }^{-5} \mathrm{~F}_{2}(0.17,0.33), 0.83,1.00,1.17,1.33$.

${ }^{3} \mathrm{D}_{2}-5 \mathrm{~F}_{3}(0.00,0.08,0.17), 1.08,1.17,1.25,1.33,1.42$.

${ }^{3} \mathrm{D}_{3}{ }^{-5} \mathrm{~F}_{2}(0.00,0.33,0.67), 0.67,1.00 .1 .33,1.67,2.00$.

${ }^{3} \mathrm{D}_{3}{ }^{-5} \mathrm{~F}_{3}(0.08,0.17,0.25), 1.08,1.17,1.25,1.33,1.42,1.50$.

${ }^{3} \mathrm{D}_{3}-5 \mathrm{~F}_{4}(0.00,0.02,0.03,0.05), 1.30,1.32,1.33,1.35,1.37,1.38,1.40$.

${ }^{3} \mathrm{D}_{1}{ }^{-5} \mathrm{G}_{2}^{\prime}(0.00,0.17), 0.17,0.33,0.50$.

${ }^{3} \mathrm{D}_{2}-5 \mathrm{G}_{2}^{\prime}(0.83,1.67),-0.50,+0.33,1.17,2.00$.

${ }^{3} \mathrm{D}_{2}{ }^{-5} \mathrm{G}^{\prime}{ }_{3}(0.00,0.25,0.50), 0.42,0.67,0.92,1.17,1.42$.

${ }^{8} \mathrm{D}_{3}{ }^{-6} \mathrm{G}_{2}^{\prime}{ }_{2}(0.00,1.00,2.00),-0.67,+0.33,1.33,2.33,3.33$.

${ }^{3} \mathrm{D}_{3}{ }^{-5} \mathrm{G}^{\prime}{ }_{3}(0.42,0.83,1.25), 0.08,0.50,0.92,1.33,1.75,2.17$.

${ }^{3} \mathrm{D}_{3}-5 \mathrm{G}^{\prime}{ }_{4}(0.00,0.18,0.37,0.55), 0.60,0.78,0.97,1.15,1.33,1.52,1.70$.

${ }^{3} \mathrm{~F}_{2}-{ }^{5} \mathrm{P}_{1}^{\prime}(0.00,1.83),-1.1 \%,+0.67,2.50$.

${ }^{3} \mathrm{~F}_{2}-5 \mathrm{P}_{2}^{\prime}(1.17,2.33),-0.50,+0.67,1.83,3.00$.

${ }^{3} \mathrm{~F}_{2}-{ }^{-5} \mathrm{P}_{3}^{\prime}(0.00,1.00,2.00),-0.33,+0.67,1.67,2.67,3.67$.

${ }^{3} \mathrm{~F}_{3}-5 \mathrm{P}^{\prime}{ }_{2}(0.00,0.75,1.50),-0.42,+0.33,1.08,1.83,2.58$.

${ }^{3} \mathrm{~F}_{3}-{ }^{-5} \mathrm{P}_{3}^{\prime}(0.58,1.17,1.75),-0.08,+0.50,1.08,1.67,2.25,2.83$.

${ }^{3} \mathrm{~F}_{4}-\mathrm{P}^{-} \mathrm{P}_{3}(0.00,0.42,0.83,1.25), 0.00,0.42,0.83,1.25,1.67,2.08,2.50$.

${ }^{3} \mathrm{~F}_{2}-{ }^{-6} \mathrm{D}_{1}(0.00,0.83),-0.17,+0.67,1.50$.

${ }^{3} \mathrm{~F}_{2}{ }^{-6} \mathrm{D}_{2}(0.83,1.67),-0.17,+0.67,1.50,2.33$.

${ }^{3} \mathrm{~F}_{2}{ }^{-5} \mathrm{D}_{3}(0.00,0.83,1.67),-0.17,+0.67,1.50,2.33,3.17$.

${ }^{3} \mathrm{~F}_{3}-{ }^{-5} \mathrm{D}_{2}(0.00,0.42,0.83), 0.25,0.67,1.08,1.50,1.92$.

${ }^{3} \mathrm{~F}_{3}-{ }^{5} \mathrm{D}_{3}(0.42,0.83,1.25), 0.25,0.67,1.08,1.50,1.92,2.33$.

${ }^{3} \mathrm{~F}_{3}-{ }^{-5} \mathrm{D}_{4}(0.00,0.42,0.83,1.25), 0.25,0.67,1.08,1.50,1.92,2.33,2.75$.

${ }^{3} \mathrm{~F}_{4}-{ }^{5} \mathrm{D}_{3}(0.00,0.25,0.50,0.75), 0.50,0.75,1.00,1.25,1.50,1.75,2.00$.

${ }^{3} \mathrm{~F}_{4} \mathrm{C}^{-5} \mathrm{D}_{4}(0.25,0.50,0.75,1.60), 0.50,0.75,1.00,1.25,1.50,1.75,2.00,2.25$.

${ }^{3} \mathrm{~F}_{2}-5 \mathrm{~F}^{\prime}{ }_{1}(0.00,0.67), 0.00,0.67,1.33$.

${ }^{3} \mathrm{~F}_{2}-{ }^{-5} \mathrm{~F}_{2}^{\prime}(0.33, \mathbf{0 . 6 7}), 0.33, \mathbf{0 . 6 7}, \mathbf{1 . 0 0}, 1.33$.

${ }^{3} \mathrm{~F}_{2}-5 \mathrm{~F}^{\prime}{ }_{3}(0.00,0.58,1.17), 0.08,0.67,1.25,1.83,2.42$.

${ }^{3} \mathrm{~F}_{3}-{ }^{-5} \mathrm{~F}_{2}^{\prime}(0.00,0.08,0.17), 0.92,1.00,1.08,1.17,1.25$.

${ }^{3} \mathrm{~F}_{3}-5 \mathrm{~F}^{\prime}{ }_{3}(0.17,0.33,0.50), 0.75,0.92, \mathbf{1 . 0 8}, \mathbf{1 . 2 5}, 1.42,1.58$.

${ }^{3} \mathrm{~F}_{3}-5 \mathrm{~F}_{4}^{\prime}(0.00,0.27,0.53,0.80), 0.55,0.82,1.08,1.35,1.62,1.88,2.15$.

${ }^{3} \mathrm{~F}_{4}-5 \mathrm{~F}^{\prime}{ }_{3}(0.00), 1.25$.

${ }^{3} \mathrm{~F}_{4}-5 \mathrm{~F}_{4}^{\prime}(0.10,0.20,0.30,0.40), 0.95,1.05,1.15,1.25,1.35,1.45,1.55,1.65$.

${ }^{3} \mathrm{~F}_{4}-5 \mathrm{~F}_{5}^{\prime}(0.00,0.15,0.30,0.45,0.60), 0.80,0.95,1.10,1.25,1.40,1.55,1.70,1.85,2.00$. 
TABLE 12.-Theoretical Zeeman effects (triplet-quintet intersystem)-Continued

${ }^{3} \mathrm{~F}_{2}{ }^{-5} \mathrm{G}_{2}(0.33,0.67), 0.00,0.33,0.6 \%, 1.00$.

${ }^{3} \mathrm{~F}_{2}-5 \mathrm{G}_{3}(0.00,0.25,0.50), 0.42,0.67,0.92,1.17,1.42$.

${ }^{3} \mathrm{~F}_{3}-\mathrm{G}_{2}(\vartheta .00,0.75,1.50),-0.42,+0.33,1.08,1.83, \mathbf{2 . 5 8}$.

${ }^{3} \mathrm{~F}_{3}-{ }^{5} \mathrm{G}_{3}(0.17,0.33,0.50), 0.58,0.75,0.92,1.08,1.25,1.42$.

${ }^{3} \mathrm{~F}_{3}-{ }^{5} \mathrm{G}_{4}(0.00,0.07,0.13,0.20), 0.95,1.02,1.08,1.15,1.22,1.28,1.35$.

${ }^{3} \mathrm{~F}_{4}{ }^{-5} \mathrm{G}_{3}(0.00,0.33,0.67,1.00), 0.25,0.58,0.92,1.25,1.58,1.92,2.25$.

${ }^{3} \mathrm{~F}_{4}{ }^{-5} \mathrm{G}_{4}(0.10,0.20,0.30,0.40), 0.85,0.95,1.05,1.15,1.25,1.35,1.45,1.55$.

${ }^{3} \mathrm{~F}_{4}{ }^{-5} \mathrm{G}_{5}(0.00,0.02,0.03,0.05,0.07), 1.20,1.22,1.23,1.25,1.27,1.28,1.30,1.32,1.33$.

${ }^{3} \mathrm{~F}_{2}-{ }^{-5} \mathrm{H}_{3}{ }_{3}(0.00,0.17,0.33), 0.17,0.33,0.50,0.67,0.83$.

${ }^{3} \mathrm{~F}_{3}-{ }^{-5} \mathrm{H}_{3}{ }_{3}(0.58,1.17,1.85),-0.67,-0.08,+0.50,1.08,1.67,2.25$.

${ }^{3} \mathrm{~F}_{3}-{ }^{5} \mathrm{H}_{4}^{\prime}(0.00,0.18,0.37,0.55), 0.35,0.53,0.72,0.90,1.08,1.27,1.45$.

${ }^{8} \mathrm{~F}_{4}{ }^{-5} \mathrm{H}_{3}^{\prime}(0.00,0.75,1.50,2.25),-1.00,-0.25,+0.50,1.25,2.00,2.75,3.50$.

${ }^{3} \mathrm{~F}_{4}{ }^{-5} \mathrm{H}^{\prime}{ }_{4}(0.35,0.70,1.05,1.40),-0.15,+0.20,0.55,0.90,1.25,1.60,1.95,2.30$.

${ }^{3} \mathrm{~F}_{4}-{ }^{5} \mathrm{~F}^{\prime}{ }_{5}(\mathbf{0 . 0 0}, 0.15,0.30,0.45,0.60), 0.50,0.65,0.80,0.95,1.10,1.25,1.40,1.55,1.70$.

${ }^{3} \mathrm{G}_{3}-{ }^{-5} \mathrm{D}_{2}^{\prime}(\boldsymbol{\theta} .00,0.75,1.50),-0.75,0.00,0.75,1.50,2.25$.

${ }^{3} \mathrm{G}_{3}-{ }^{5} \mathrm{D}^{\prime}{ }_{3}(0.75,1.50,2.25),-0.75,0.00,0.75,1.50,2.25,3.00$.

${ }^{3} \mathrm{G}_{3}-6 \mathrm{D}_{4}^{\prime}(0.00,0.75,1.50,2.25)-0.75,0.00,0.75,1.50,2.25,3.00,3.75$.

${ }^{3} \mathrm{G}_{4-5} \mathrm{D}_{3}^{\prime}(0.00,0.45,0.90,1.35),-0.30,+0.15,0.60,1.05,1.50,1.95,2.40$.

${ }_{3} \mathrm{G}_{4}-5 \mathrm{D}_{4}^{\prime}(0.45,0.90,1.35,1.80),-0.30,+0.15,0.60,1.05,1.50,1.95,2.40,2.85$.

${ }^{3} \mathrm{G}_{5^{-5}} \mathrm{D}_{4}(0.00,0.30,0.60,0.90,1.20), 0.00,0.30,0.60,0.90,1.20,1.50,1.80,2.10,2.40$.

${ }^{3} \mathrm{G}_{3}-{ }^{5} \mathrm{~F}_{2}(0.00,0.25,0.50), 0.25,0.50,0.75,1.00,1.25$.

${ }^{3} \mathrm{G}_{3}-{ }^{-5} \mathrm{~F}_{3}(0.50,1.00,1.50),-0.25,+0.25, \mathbf{0 . 7 5}, \mathbf{1 . 2 5}, 1.75,2.25$.

${ }^{3} \mathrm{G}_{3}{ }^{-5} \mathrm{~F}_{4}(0.00,0.60,1.20,1.80),-0.45,+0.15,0.75,1.35,1.95,2.55,3.15$.

${ }^{3} \mathrm{G}_{4}-5 \mathrm{~F}_{3}(0.00,0.20,0.40,0.60), 0.45,0.65,0.85,1.05,1.25,1.45,1.65$,

${ }^{3} \mathrm{G}_{4^{-}-5} \mathrm{~F}_{4}(0.30,0.60,0.90, \mathbf{1 . 2 0}), 0.15,0.45,0.75, \mathbf{1 . 0 5}, \mathbf{1 . 3 5}, 1.65,1.95,2.25$.

${ }^{3} \mathrm{G}_{4}-5 \mathrm{~F}_{5}(0.00,0.35,0.70,1.05,1.40), 0.00,0.35,0.70,1.05,1.40,1.75,2.10,2.45$, 2.80.

${ }^{3} \mathrm{G}_{5}-5 \mathrm{~F}_{4}(0.00,0.15,0.30,0.45,0.60), 0.60,0.75,0.90,1.05,1.20,1.35,1.50,1.65,1.80$.

${ }^{3} \mathrm{G}_{5}{ }^{-5} \mathrm{~F}_{5}(0.20,0.40,0.60,0.80,1.00), 0.40,0.60,0.80,1.00,1.20,1.40,1.60,1.80,2.00,2.20$.

${ }^{3} \mathrm{G}_{3}-5 \mathrm{G}_{2}{ }_{2}(\mathbf{0 . 0 0}, 0.42,0.83),-0.08,+0.33,0.75,1.17,1.58$.

${ }^{3} \mathrm{G}_{3}-{ }^{-5} \mathrm{G}^{\prime}{ }_{3}(0.17,0.33,0.50), 0.42,0.58,0.75,0.92,1.08,1.25$.

${ }^{3} \mathrm{G}_{3}-5 \mathrm{G}^{\prime}{ }_{4}(0.00,0.40,0.80,1.20),-0.05,+0.35,0.75,1.15,1.55,1.95,2.35$.

${ }^{3} \mathrm{G}_{4}-{ }^{5} \mathrm{G}^{\prime}{ }_{3}(\mathbf{0 . 0 0}, 0.13,0.27,0.40), 0.65,0.78,0.92,1.05,1.18,1.32, \mathbf{1 . 4 5}$.

${ }^{3} \mathrm{G}_{4}-{ }^{-5} \mathrm{G}^{\prime}{ }_{4}(0.10,0.20,0.30,0.40), 0.75,0.85,0.95,1.05,1.15,1.25,1.35,1.45$.

${ }^{3} \mathrm{G}_{4^{-5}} \mathrm{G}^{\prime}{ }_{5}(0.00,0.22,0.43,0.65,0.87), 0.40,0.62,0.83,1.05,1.27,1.48,1.70,1.92,2.13$.

${ }^{3} \mathrm{G}_{5}-5 \mathrm{G}^{\prime}{ }_{4}(0.00,0.05,0.10,0.15,0.20), 1.00,1.05,1.10,1.15,1.20,1.25,1.30,1.35,1.40$.

${ }^{3} \mathrm{G}_{5}-5 \mathrm{G}^{\prime}{ }_{5}(0.07,0.13,0.20,0.27,0.33), 0.93,1.00,1.07,1.13,1.20,1.2 \%, 1.33,1.40,1.47,1.53$.

${ }^{3} \mathrm{G}_{5}-{ }^{5} \mathrm{G}^{\prime}{ }_{6}(0.00,0.13,0.27,0.40,0.53,0.67), 0.67,0.80,0.93,1.07,1.20,1.33,1.47,1.60,1.73,1.87,2.00$.

${ }^{3} \mathrm{G}_{3}-5 \mathrm{H}_{3}(0.25,0.50,0.75), 0.00,0.25,0.50,0.75,1.00,1.25$.

${ }^{3} \mathrm{G}_{3}-{ }^{5} \mathrm{H}_{4}(0.00,0.15,0.30,0.45), 0.45,0.60,0.75,0.90,1.05,1.20,1.35$.

${ }^{3} \mathrm{G}_{4}-{ }^{-5} \mathrm{H}_{3}(0.00,0.55,1.10,1.65),-0.60,-0.05,+0.50,1.05,1.60,2.15$, 2.70.

${ }^{3} \mathrm{G}_{4}-5 \mathrm{H}_{4}(0.15,0.30,0.45,0.60), 0.45,0.60,0.75,0.90,1.05,1.20,1.35,1.50$.

${ }^{3} \mathrm{G}_{4}-{ }^{5} \mathrm{H}_{5}(\mathbf{0 . 0 0}, 0.05,0.10,0.15,0.20), 0.90,0.95,1.00,1.05,1.10,1.15,1.20,1.25,1.30$.

${ }^{3} \mathrm{G}_{5}-{ }^{-5} \mathrm{H}_{4}(0.00,0.30,0.60,0.90,1.20), 0.00,0.30,0.60,0.90,1.20,1.50,1.80,2.10,2.40$.

${ }^{8} \mathrm{G}_{5}-{ }^{5} \mathrm{H}_{5}(0.10,0.20,0.30,0.40,0.50), 0.70,0.80,0.90,1.00,1.10,1.20,1.30,1.40,1.50,1.60$.

${ }^{3} \mathrm{G}_{5}-{ }^{5} \mathrm{H}_{6}(0.00,0.01,0.03,0.04,0.06,0.07), 1.14,1.16,1.17,1.19,1.20,1.21,1.23,1.24,1.26,1.27,1.29$ 。

${ }^{3} \mathrm{G}_{8}-5 \mathrm{I}^{\prime}{ }_{4}(0.09,0.15,0.30,0.45), 0.15,0.30,0.45,0.60,0.75,0.90,1.05$.

${ }^{3} \mathrm{G}_{4}-5 \mathrm{I}_{4}^{\prime}(0.45,0.90,1.35,1.80),-0.75,-0.30,+0.15,0.60,1.05,1.50,1.95,2.40$.

${ }^{3} \mathrm{G}_{4}-5 \mathrm{I}^{\prime}{ }_{5}(0.00,0.15,0.30,0.45,0.60), 0.30,0.45,0.60,0.75,0.90,1.05,1.20,1.35,1.50$.

${ }^{3} \mathrm{G}_{5}-5 \mathrm{I}^{\prime}{ }_{4}(0.00,0.60,1.20,1.80,2.40),-1.20,-0.60,0.00,0.60,1.20,1.80,2.40,3.00,3.60$.

${ }^{3} \mathrm{G}_{5}-5 \mathrm{I}_{5}^{\prime}{ }_{5}(0.30,0.60,0.90,1.20,1.50),-0.30,0.00,0.30,0.60,0.90,1.20,1.50,1.80,2.10,2.40$.

${ }^{3} \mathrm{G}_{5}{ }^{-5} \mathrm{I}_{6}{ }_{6}(0.00,0.13,0.26,0.38,0.51,0.64), 0.43,0.56,0.69,0.81,0.94,1.07,1.20,1.33,1.46,1.59,1.72$.

${ }^{3} \mathrm{H}_{4}-5 \mathrm{~F}^{\prime}{ }_{3}(0.00,0.45,0.90,1.35),-0.55,-0.10,+0.35,0.80,1.25,1.70,2.15$.

${ }^{3} \mathrm{H}_{4}-5 \mathrm{~F}^{\prime}{ }_{4}(0.55,1.10,1.65,2.20),-0.85,-0.30,+0.25,0.80,1.35,1.90,2.45,3.00$.

${ }^{3} \mathrm{H}_{4}-5 \mathrm{~F}^{\prime}{ }_{5}(0.00,0.60,1.20,1.80,2.40),-1.00,-0.40,+0.20,0.80,1.40,2.00,2.60,3.20,3.80$.

${ }^{3} \mathrm{H}_{5}-5 \mathrm{~F}^{\prime}{ }_{4}(\mathbf{0 . 0 0}, 0.31,0.63,0.95,1.27),-0.23,+0.08,0.40,0.72,1.03,1.35,1.67,1.98,2.30$.

${ }^{3} \mathrm{H}_{5}-5 \mathrm{~F}^{\prime}{ }_{5}(0.37,0.73,1.10,1.47,1.83),-0.43,-0.07,+0.30,0.67,1.03,1.40,1.77,2.13,2.50,2.87$.

${ }^{3} \mathrm{H}_{6}-{ }^{-5} \mathrm{~F}_{5}{ }_{5}(0.00,0.23,0.47,0.70,0.93,1.17), 0.00,0.23,0.47,0.70,0.93,1.17,1.40,1.63,1.87,2.10,2.33$.

${ }^{3} \mathrm{H}_{4}-5 \mathrm{G}_{3}(0.00,0.12,0.23,0.35), 0.45,0.57,0.68,0.80,0.92,1.03,1.15$.

${ }^{3} \mathrm{H}_{4}-5 \mathrm{G}_{4}(0.35,0.70,1.05,1.40),-0.25,+0.10,0.45,0.30,1.15,1.50,1.85,2.20$.

${ }^{3} \mathrm{H}_{4}-5 \mathrm{G}_{5}(\mathbf{0 . 0 0}, 0.47,0.93,1.40,1.87),-0.60,-0.13,+0.33,0.80,1.27,173,2.20,2.67,3.13$.

${ }^{3} \mathrm{H}_{5}{ }^{-5} \mathrm{G}_{4}(0.00,0.12,0.23,0.35,0.47), 0.57,0.68,0.80,0.92,1.03,1.15,1.27,1.38,1.50$.

${ }^{3} \mathrm{H}_{5}-5 \mathrm{G}_{5}(0.23,0.47,0.70,0.93,1.17), 0.10,0.33,0.57,0.80,1.03,1.27,1.50,1.73,1.97,2.20$. 
TABLE 12.-Theoretical Zeeman effects (triplet-quintet intersystem)-Continued

${ }^{3} \mathrm{H}_{5^{-5}} \mathrm{G}_{6}(0.00,0.30,0.60,0.90,1.20,1.50),-0.17,+0.13,0.43,0.73,1.03,1.33,1.63,1.93,2.23,2.53,2.83$. ${ }^{3} \mathrm{H}_{6^{-5}} \mathrm{G}_{5}(0.00,0.10,0.20,0.30,0.40,0.50), 0.67,0.77,0.87,0.97,1.07,1.17,1.27,1.37,1.47,1.57,1.67$. ${ }^{3} \mathrm{H}_{6^{-5}} \mathrm{G}_{6}(0.17,0.33,0.50,0.67,0.83,1.00), 0.33,0.50,0.67,0.83,1.00,1.17,1.33,1.50,1.67,1.83,2.00,2.17$.

${ }^{3} \mathrm{H}_{4}{ }^{-5} \mathrm{H}_{3}{ }_{3}(0.00,0.30,0.60,0.90),-0.10,+0.20,0.50,0.80,1.10,1.40,1.70$.

${ }^{3} \mathrm{H}_{4}{ }^{-5} \mathrm{H}_{4}^{\prime}(0.10,0.20,0.30,0.40), 0.50,0.60,0.70,0.80,0.90,1.00,1.10,1.20$.

${ }^{3} \mathrm{H}_{4}{ }^{-5} \mathrm{H}^{\prime}{ }_{5}(0.00,0.30,0.60,0.90,1.20),-0.10,+0.20,0.50,0.80,1.10,1.40,1.70,2.00,2.30$.

${ }^{3} \mathrm{H}_{5}-5 \mathrm{H}^{\prime}{ }_{4}(0.00,0.13,0.27,0.40,0.53), 0.50,0.63,0.77,0.90,1.03,1.17,1.30,1.43,1.5 \%$.

${ }^{3} \mathrm{H}_{5}-5 \mathrm{H}_{5}^{\prime}(0.07,0.13,0.20,0.27,0.33), 0.77,0.83,0.90,0.97,1.03,1.10,1.17,1.23,1.30,1.37$.

${ }^{3} \mathrm{H}_{5}{ }^{-5} \mathrm{H}_{6}{ }_{6}(0.00,0.18,0.36,0.54,0.72,0.90), 0.31,0.49,0.67,0.85,1.03,1.21,1.40,1.58,1.76,1.94,2.12$.

${ }^{3} \mathrm{H}_{6}{ }^{-5} \mathrm{H}_{5}^{\prime}(0.00,0.07,0.13,0.20,0.27,0.33), 0.83,0.90,0.97,1.03,1.10,1.17,1.23,1.30,1.37,1.43,1.50$.

${ }^{3} \mathrm{H}_{6}{ }^{-5} \mathrm{H}^{\prime}{ }_{6}(0.05,0.10,0.14,0.19,0.24,0.29), 0.93,0.98,1.02,1.07,1.12,1.17,1.21,1.26,1.31,1.36,1.40,1.45$.

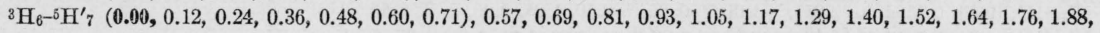
2.00.

${ }^{3} \mathrm{H}_{4}-5 \mathrm{I}_{4}(0.20,0.40,0.60,0.80), 0.00,0.20,0.40,0.60,0.80,1.00,1.20,1.40$.

${ }^{3} \mathrm{H}_{4^{-5} \mathrm{I}_{5}}(\mathbf{0 . 0 0}, 0.10,0.20,0.30,0.40), 0.50,0.60,0.70,0.80,0.90,1.00,1.10,1.20,1.30$.

${ }^{3} \mathrm{H}_{5}-5 \mathrm{I}_{4}(0.00,0.43,0.87,1.30,1.73),-0.70,-0.27,+0.17,0.60,1.03,1.47,1.90,2.34$.

${ }^{3} \mathrm{H}_{5}{ }^{-5} \mathrm{I}_{5}(0.13,0.27,0.40,0.53,0.67), 0.37,0.50,0.63,0.77,0.90,1.03,1.17,1.30,1.43,1.57$.

${ }^{3} \mathrm{H}_{5}-5 \mathrm{I}_{6}(0.00,0.04,0.08,0.11,0.15,0.19), 0.88,0.92,0.96,0.99,1.03,1.07,1.11,1.15,1.18,1.22,1.26$.

${ }^{3} \mathrm{H}_{6}{ }^{-5} \mathrm{I}_{5}(0.00,0.27,0.53,0.80,1.07,1.33),-0.17,+0.10,0.37,0.63,0.90,1.17,1.43,1.70,1.97,2.23,2.50$.

${ }^{3} \mathrm{H}_{6}{ }^{-5} \mathrm{I}_{6}(0.10,0.19,0.29,0.38,0.48,0.57), 0.60,0.69,0.79,0.88,0.98,1.07,1.17,1.27,1.36,1.45,1.55,1.64$.

${ }_{3} \mathrm{H}_{0}{ }^{-5} \mathrm{I}_{7}(0.00,0.01,0.02,0.04,0.05,0.06,0.07), 1.11,1.12,1.13,1.14,1.16,1.17,1.18,1.19,1.20,1.22,1.23,1.24$, 1.25 .

\section{TABLE 13.--Theoretical Zeeman effects (quintet-septet intersystem)}

${ }^{8} \mathrm{~S}_{2}-{ }^{7} \mathrm{~S}_{3}(0.00), 2.00$.

${ }^{5} \mathrm{~S}_{2}-7 \mathrm{P}_{2}(0.33,0.67), 1.67,2.00,2.33,2.67$.

${ }^{5} \mathrm{~S}_{2}-7 \mathrm{P}_{3}(0.00,0.08,0.17), 1.75,1.83,1.92,2.00,2.08$.

${ }^{{ }^{5} \mathrm{~S}_{2}-7} \mathrm{D}_{1}^{\prime}(0.00,1.00), \mathbf{1 . 0 0}, 2.00,3.00$.

${ }^{5} \mathrm{~S}_{2}-{ }^{7} \mathrm{D}^{\prime}{ }_{2}(0.00), 2.00$.

${ }^{5} \mathrm{~S}_{2}-7 \mathrm{D}^{\prime}{ }_{3}(0.00,0.25,0.50), 1.25,1.50,1.75,2.00,2.25$.

${ }^{5} \mathrm{P}_{2}{ }^{7} \mathrm{~S}_{3}(0.09,0.17,0.33), 1.67,1.83,2.00,2.17,2.33$.

${ }^{8} \mathrm{P}_{3}{ }^{-7} \mathrm{~S}_{3}(0.33,0.67,1.00), 1.00,1.33,1.67,2.00,2.33,2.67$.

${ }^{5} \mathrm{P}_{1^{-7}} \mathrm{P}_{2}^{\prime}(0.00,0.17), 2.17,2,33,2.50$.

${ }^{5} \mathrm{P}_{2}{ }^{7} \mathrm{P}^{\prime}{ }_{2}(0.50,1.00), 1.33,1.83,2.33,2.83$.

${ }^{5} \mathrm{P}_{2}{ }^{7} \mathrm{P}_{3}^{\prime}{ }_{3}(0.00,0.08,0.17), 1.75,1.83,1.92,2.00,2.08$.

${ }^{5} \mathrm{P}_{3}-7 \mathrm{P}_{2}^{\prime}{ }_{2}(0.00,0.67,1.33), 0.33,1.00,1.67,2.33,3.00$.

${ }^{5} \mathrm{P}_{3}{ }^{7} \mathrm{P}^{\prime}{ }_{3}(0.25,0.50,0.75), 1.17,1.42,1.67,1.92,2.17,2.42$.

${ }^{5} \mathrm{P}_{3}-7 \mathrm{P}_{4}^{\prime}(0.00,0.08,0.17,0.25), 1.50,1.58,1.67,1.75,1.83,1.92,2.60$.

${ }^{8} \mathrm{P}_{1}-7 \mathrm{D}_{1}(0.50), 2.50,3.00$.

${ }^{6} \mathrm{P}_{1}{ }^{7} \mathrm{D}_{2}(0.00,0.50), 1.50,2.00,2.50$.

${ }^{5} \mathrm{P}_{2}{ }^{-7} \mathrm{D}_{1}(\mathbf{0 . 0 0}, 1.17), \mathbf{0 . 6 7}, 1.83,3.00$.

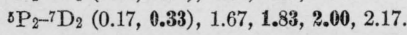

${ }^{5} \mathrm{P}_{2}{ }^{7} \mathrm{D}_{3}(0.00,0.08,0.17), 1.58,1.67,1.75,1.83,1.92$.

${ }^{5} \mathrm{P}_{3}{ }^{7} \mathrm{D}_{2}(0.00,0.33,0.67), 1.00,1.33,1.67,2.00 .2 .33$.

${ }^{5} \mathrm{P}_{3}{ }^{7} \mathrm{D}_{3}(0.08,0.17, \mathbf{0 . 2 5}), 1.50,1.58, \mathbf{1 . 6 7}, 1.75,1.83,1.92$.

${ }^{5} \mathrm{P}_{3}-7 \mathrm{D}_{4}(0.00,0.02,0.03,0.05), 1.60,1.62,1.63,1.65,1.67,1.68,1.70$.

${ }^{8} \mathrm{P}_{1}{ }^{7} \mathrm{~F}^{\prime}{ }_{0}(0.00), 2.50$.

${ }^{5} \mathrm{P}_{1}-7 \mathrm{~F}^{\prime}{ }_{1}(1.00), 1.50,2.50$.

${ }^{8} \mathrm{P}_{1}-7 \mathrm{~F}^{\prime}{ }_{2}(0.00,1.00), 0.50,1.50,2.50$.

${ }^{5} \mathrm{P}_{2}-7 \mathrm{~F}^{\prime}{ }_{1}(0.00,0.33), 1.50,1.83,2.1 \%$.

${ }^{5} \mathrm{P}_{2}-7 \mathrm{~F}^{\prime}{ }_{2}(0.33,0.67), 1.17,1.50,1.83,2.17$.

${ }^{3} \mathrm{P}_{2}-7 \mathrm{~F}^{\prime}{ }_{3}(\boldsymbol{\theta} .00,0.33,0.67), 0.83,1.17,1.50,1.83,2.17$.

${ }^{8} \mathrm{P}_{3}-7 \mathrm{~F}^{\prime}{ }_{2}(0.00,0.17,0.33), 1.33,1.50,1.67,1.83,2.09$.

${ }^{8} \mathrm{P}_{3}{ }^{7} \mathrm{~F}^{\prime}{ }_{3}(0.17,0.33,0.50), 1.17,1.33, \mathbf{1 . 5 0}, \mathbf{1 . 6 7}, 1.83,2.00$.

${ }^{5} \mathrm{P}_{3}-7 \mathrm{~F}_{4}^{\prime}{ }_{4}(0.00,0.17,0.33,0.50), 1.00,1.17,1.33,1.50,1.67,1.83,2.00$.

${ }^{5} \mathrm{D}_{2}-7 \mathrm{~S}_{3}^{\prime}(0.00,0.50,1.00), 1.00,1.50,2.00,2.50,3.00$.

${ }^{5} \mathrm{D}_{3}-{ }^{-} \mathrm{S}^{\prime}{ }_{3}(0.50,1.00, \mathbf{1 . 5 0}), 0.50,1.00,1.50,2.00,2.50,3.00$.

${ }^{5} \mathrm{D}_{4}{ }^{-7} \mathrm{~S}^{\prime}{ }_{3}(0.00,0.50,1.00,1.50), 0.00,0.50,1.00,1.50,2.00,2.50,3.00$ 
TABLE 13.-Theoretical Zeeman effects (quintet-septet intersystem)-Continued

${ }^{5} \mathrm{D}_{1}{ }^{7} \mathrm{P}_{2}(0.00,0.83), 1.50,2.33,3.1 \%$.

${ }^{5} \mathrm{D}_{2}{ }^{-7} \mathrm{P}_{2}(0.83,1.67), 0.67,1.50,2.33,3.17$.

${ }^{8} \mathrm{D}_{2}-{ }^{-7} \mathrm{P}_{3}(0.00,0.42,0.83), 1.08,1.50,1.92,2.33,2.75$.

${ }^{8} \mathrm{D}_{3}{ }^{-7} \mathrm{P}_{2}(0.00,0.83,1.67),-0.17,+0.67,1.50,2.33,3.17$.

${ }^{8} \mathrm{D}_{3}-{ }^{-7} \mathrm{P}_{3}(0.42,0.83,1.25), 0.67,1.08,1.50,1.92,2.33,2.75$.

${ }^{8} \mathrm{D}_{3}{ }^{-7} \mathrm{P}_{4}(0.00,0.25,0.50,0.75), 1.00,1.25,1.50,1.75,2.00,2.25,2.50$.

${ }^{6} \mathrm{D}_{4}{ }^{-7} \mathrm{P}_{3}(0.00,0.42,0.83,1.25), 0.25,0.67,1.08,1.50,1.92,2.33,2.75$.

${ }^{5} \mathrm{D}_{4}-7 \mathrm{P}_{4}(0.25,0.50,0.75,1.00), 0.75,1.00,1.25,1.50,1.75,2.00,2.25,2.50$.

${ }^{8} \mathrm{D}_{0}{ }^{-7} \mathrm{D}^{\prime}{ }_{1}(0.00), 3.00$.

${ }^{5} \mathrm{D}_{1}{ }^{7} \mathrm{D}^{\prime}{ }_{1}(1.50), 1.50,3.00$.

${ }^{5} \mathrm{D}_{1}{ }^{-7} \mathrm{D}_{2}^{\prime}(\mathbf{0 . 0 0}, 0.50), 1.50,2.00,2.50$.

${ }^{8} \mathrm{D}_{2}-7 \mathrm{D}^{\prime}{ }_{1}(0.00,1.50), 0.00,1.50,3.00$.

${ }^{8} \mathrm{D}_{2}-{ }^{7} \mathrm{D}^{\prime}{ }_{2}(0.50,1.00), 1.00,1.50,2.00,2.50$.

${ }^{8} \mathrm{D}_{2}-{ }^{7} \mathrm{D}^{\prime}{ }_{3}(0.00,0.25,0.50), 1.25,1.50,1.75,2.00,2.25$.

${ }^{5} \mathrm{D}_{3}-7 \mathrm{D}^{\prime}{ }_{2}(0.00,0.50,1.00), 0.50,1.00,1.50,2.00,2.50$.

${ }^{8} \mathrm{D}_{3}-7 \mathrm{D}^{\prime}{ }_{3}(0.25,0.50,0.75), 1.00,1.25,1.50,1.75,2.00,2.25$.

${ }^{8} \mathrm{D}_{3}-7 \mathrm{D}_{4}^{\prime}(\mathbf{0 . 0 0}, 0.15,0.30,0.45), 1.20,1.35,1.50,1.65,1.80,1.95,2.10$.

${ }^{8} \mathrm{D}_{4}{ }^{7} \mathrm{D}_{3}^{\prime}{ }_{3}(\mathbf{0 . 0 0}, 0.25,0.50,0.75), 0.75,1.00,1.25,1.50,1.75,2.00,2.25$.

${ }^{8} \mathrm{D}_{4-7}{ }^{7} \mathrm{D}_{4}^{\prime}(0.15,0.30,0.45,0.60), 1.05,1.20,1.35,1.50,1.65,1.80,1.95,2.10$.

${ }^{5} \mathrm{D}_{4^{-}-7} \mathrm{D}^{\prime}{ }_{5}(\mathbf{0 . 0 0}, 0.10,0.20,0.30,0.40), 1.20,1.30,1.40,1.50,1.60,1.70,1.80,1.90,2.00$.

${ }^{8} \mathrm{D}_{0}{ }^{7} \mathrm{~F}_{1}={ }^{5} \mathrm{D}_{1}{ }^{-7} \mathrm{~F}_{0}={ }^{7} \mathrm{D}_{1}{ }^{7} \mathrm{~F}_{1}={ }^{5} \mathrm{D}_{1}{ }^{7} \mathrm{~F}_{2}={ }^{5} \mathrm{D}_{2}{ }^{7} \mathrm{~F}_{1}={ }^{5} \mathrm{D}_{2}-7 \mathrm{~F}_{2}={ }^{5} \mathrm{D}_{2}-7 \mathrm{~F}_{3}=\ldots={ }^{5} \mathrm{D}_{4}{ }^{7} \mathrm{~F}_{5}(0.00), 1.50$.

${ }^{8} \mathrm{D}_{0}{ }^{7} \mathrm{G}^{\prime}{ }_{1}(0.00), 0.50$.

${ }^{8} \mathrm{D}_{1}-{ }^{7} \mathrm{G}^{\prime}{ }_{1}(2.00),-0.50,+1.50$.

${ }^{8} \mathrm{D}_{1}{ }^{7} \mathrm{G}^{\prime}{ }_{2}(0.00,0.67), 0.17,0.83,1.50$.

${ }^{8} \mathrm{D}_{2}-7 \mathrm{G}^{\prime}{ }_{1}(0.00,2.00),-0.50,+1.50,3.50$.

${ }^{8} \mathrm{D}_{2}-{ }^{7} \mathrm{G}_{2}{ }_{2}(0.67,1.33), 0.17,0.83,1.50,2.17$.

${ }^{8} \mathrm{D}_{2}-{ }^{-7} \mathrm{G}^{\prime}{ }_{3}(\mathbf{0 . 0 0}, 0.33,0.67), \mathbf{0 . 5 0}, 0.83,1.17,1.50,1.83$.

${ }^{B} \mathrm{D}_{3}-7 \mathrm{G}_{2}{ }_{2}(0.00,0.67,1.33), 0.17,0.83,1.50,2.17,2.83$.

${ }^{8} \mathrm{D}_{3}{ }^{-7} \mathrm{G}_{3}{ }_{3}(0.33,0.67,1.00), 0.50,0.83,1.17,1.50,1.83,2.17$.

${ }^{8} \mathrm{D}_{3}-^{7} \mathrm{G}_{4}{ }_{4}(0.00,0.20,0.40,0.60), 0.70,0.90,1.10,1.30,1.50,1.70,1.90$.

${ }^{3} \mathrm{D}_{4}-7 \mathrm{G}_{3}{ }_{3}(\mathbf{0 . 0 0}, 0.33,0.67,1.00), 0.50,0.83,1.17,1.50,1.83,2.17,2.50$.

${ }^{8} \mathrm{D}_{4}{ }^{-7} \mathrm{G}_{4}^{\prime}(0.20,0.40,0.60,0.80), 0.70,0.90,1.10,1.30,1.50,1.70,1.90,2.10$.

${ }^{5} \mathrm{D}_{4}{ }^{-7} \mathrm{G}^{\prime}{ }_{5}(0.00,0.13,0.27,0.40,0.53), 0.83,0.97,1.10,1.23,1.37,1.50,1.63,1.77,1.90$.

${ }^{5} \mathrm{~F}_{1}{ }^{7} \mathrm{P}_{2}{ }_{2}(0.00,2.33), 0.00,2.33,4.67$.

${ }^{5} \mathrm{~F}_{2}-7 \mathrm{P}_{2}^{\prime}(1.33,2.67),-0.33,+\mathbf{1 . 0 0}, \mathbf{2 . 3 3}, 3.67$.

${ }^{3} \mathrm{~F}_{2}-7 \mathrm{P}^{\prime}{ }_{3}(\mathbf{0 . 0 0}, 0.92,1.83), 0.08,1.00,1.92,2.83,3.75$.

${ }^{8} \mathrm{~F}_{3}-7 \mathrm{P}_{2}^{\prime}(0.00,1.08,2.17),-0.92,+0.17,1.25,2.33,3.42$.

${ }^{8} \mathrm{~F}_{3}-7 \mathrm{P}^{\prime}{ }_{3}(0.67,1.33,2.00),-0.08,+0.58,1.25,1.92,2.58,3.25$.

${ }^{5} \mathrm{~F}_{3}-7 \mathrm{P}_{4}^{\prime}(0.00,0.50,1.00,1.50), 0.25,0.75,1.25,1.75,2.25,2.75,3.25$.

${ }^{3} \mathrm{~F}_{4}-7 \mathrm{P}^{\prime}{ }_{3}(0.00,0.57,1.13,1.70),-0.35,+0.21,0.78,1.35,1.92,2.48,3.05$.

${ }^{5} \mathrm{~F}_{4}-7 \mathrm{P}^{\prime}{ }_{4}(0.40,0.80,1.20,1.60), 0.15,0.55,0.95,1.35,1.75,2.15,2.55,2.95$.

${ }^{5} \mathrm{~F}_{5} \rightarrow \mathrm{P}^{\prime}{ }_{4}(\mathbf{0 . 0 0}, 0.35,0.70,1.05,1.40), 0.00,0.35,0.70,1.05,1.40,1.75,2.10,2.45,2.80$.

${ }^{5} \mathrm{~F}_{1}-{ }^{7} \mathrm{D}_{1}(3.00), 0.00,3.00$.

${ }^{5} \mathrm{~F}_{1}-7 \mathrm{D}_{2}(\mathbf{0 . 0 0}, 2.00), 0.00,2.00,4.00$.

${ }^{8} \mathrm{~F}_{2}-i \mathrm{D}_{1}(\mathbf{0 . 0 0}, 2.00),-1.00,+1.00,3.00$.

${ }^{3} \mathrm{~F}_{2}-{ }^{-} \mathrm{D}_{2}(1.00,2.00), 0.00,1.00,2.00,3.00$.

${ }^{8} \mathrm{~F}_{2}-{ }^{7} \mathrm{D}_{3}(0.00,0.75,1.50), 0.25,1.00,1.75,2.50,3.25$.

${ }^{3} \mathrm{~F}_{3}-7 \mathrm{D}_{2}(0.00,0.75,1.50),-0.25,+0.50,1.25,2.00,2.75$.

${ }^{8} \mathrm{~F}_{3} \rightarrow \mathrm{D}_{3}(0.50,1.00,1.50), 0.25,0.75,1.25,1.75,2.25,2.75$.

${ }^{8} \mathrm{~F}_{3}-\mathrm{D}_{4}(0.00,0.40,0.80,1.20), 0.45,0.85,1.25,1.65,2.05,2.45,2.85$.

${ }^{3} \mathrm{~F}_{4}-{ }^{7} \mathrm{D}_{3}(0.00,0.40,0.80,1.20), 0.15,0.55,0.95,1.35,1.75,2.15,2.55$.

${ }^{8} \mathrm{~F}_{4}{ }^{-7} \mathrm{D}_{4}(0.30,0.60,0.90,1.20), 0.45,0.75,1.05,1.35,1.65,1.95,2.25,2.55$.

${ }^{3} \mathrm{~F}_{4}{ }^{7} \mathrm{D}_{5}(0.00,0.25,0.50,0.75,1.00), 0.60,0.85,1.10,1.35,1.60,1.85,2.10,2.35,2.60$.

${ }^{8} \mathrm{~F}_{5}-{ }^{7} \mathrm{D}_{4}(0 . \mathrm{CO}, 0.25,0.50,0.75,1.00), 0.40,0.65,0.90,1.15,1.40,1.65,1.90,2.15,2.40$.

${ }^{8} \mathrm{~F}_{5}{ }^{-} \mathrm{D}_{5}(0.20,0.40,0.60,0.80,1.00), 0.60,0.80,1.00,1.20,1.40,1.60,1.80,2.00,2.20,2.40$.

${ }^{6} \mathrm{~F}_{1}-7 \mathrm{~F}^{\prime}{ }_{0}(0.00), 0.00$ unaffected.

${ }^{\imath} \mathrm{F}_{1}-7 \mathrm{~F}^{\prime}{ }_{1}$ (1.50), 0.00, 1.50.

${ }^{8} \mathrm{~F}_{1}-7 \mathrm{~F}^{\prime}{ }_{2}(0.00,1.50), 0.00,1.50,3.00$.

${ }^{8} \mathrm{~F}_{2}-7 \mathrm{~F}^{\prime}{ }_{1}(0.00,0.50), 0.50,1.00,1.50$.

${ }^{8} \mathrm{~F}_{2}-7 \mathrm{~F}^{\prime}{ }_{2}(0.50,1.00), 0.50,1.00,1.50,2.00$.

${ }^{8} \mathrm{~F}_{2}{ }^{7} \mathrm{~F}^{\prime}{ }_{3}(0.00,0.50,1.00), 0.50,1.00,1.50,2.00,2.50$.

${ }^{8} \mathrm{~F}_{\mathbf{8}}-7 \mathrm{~F}^{\prime}{ }_{2}(0.00,0.25,0.50), 0.75,1.00,1.25,1.50,1.75$.

${ }^{5} \mathrm{~F}_{3}-{ }^{7} \mathrm{~F}^{\prime}{ }_{3}(0.25,0.50,0.75), 0.75,1.00,1.25,1.50,1.75,2.00$. 
TABLE 13.-Theoretical Zeeman effects (quintet-septet intersystem)-Continued

${ }^{5} \mathrm{~F}_{3}-7 \mathrm{~F}_{4}^{\prime}(0.00,0.25,0.50,0.75), 0.75,1.00,1.25,1.50,1.75,2.00$, 2.2.5. ${ }^{5} \mathrm{~F}_{4}-{ }^{-7} \mathrm{~F}^{\prime}{ }_{3}(0.09,0.15,0.30,0.45), 0.90,1.05,1.20,1.35,1.50,1.65,1.80$. ${ }^{3} \mathrm{~F}_{4}-7 \mathrm{~F}_{4}{ }_{4}(0.15,0.30,0.45,0.60), 0.90,1.05,1.20,1.35,1.50,1.65,1.80,1.95$.

${ }^{5} \mathrm{~F}_{4}{ }^{7} \mathrm{~F}^{\prime}{ }_{5}(0.00,0.15,0.30,0.45,0.60), 0.90,1.05,1.20,1.35,1.50,1.65,1.80,1.95,2.10$. ${ }^{5} \mathrm{~F}_{5}-7 \mathrm{~F}_{4}{ }_{4}(0.00,0.10,0.20,0.30,0.40), 1.00,1.10,1.20,1.30,1.40,1.50,1.60,1.70,1.80$.

${ }^{8} \mathrm{~F}_{5}{ }^{-7 \mathrm{~F}^{\prime}}{ }_{5}(0.10,0.20,0.30,0.40,0.50), 1.00,1.10,1.20,1.30,1.40,1.50,1.60,1.70,1.80,1.90$.

${ }^{5} \mathrm{~F}_{5}-7 \mathrm{~F}^{\prime}{ }_{6}(0.00,0.10,0.20,0.30,0.40,0.50), 1.00,1.10,1.20,1.30,1.40,1.50,1.60,1.70,1.80,1.90,2.00$.

${ }^{8} \mathrm{~F}_{1}{ }^{-7} \mathrm{G}_{1}(0.50), 0.00,0.50$.

${ }^{8} \mathrm{~F}_{1}{ }^{7}-\mathrm{G}_{2}(0.00,0.83), 0.00,0.83,1.6 \%$.

${ }^{6} \mathrm{~F}_{2}-{ }^{-7} \mathrm{G}_{1}(0.00,1.50),-0.50,+1.00$, 2.50

${ }^{5} \mathrm{~F}_{2}{ }^{-7} \mathrm{G}_{2}(0.17,0.33), 0.67,0.83,1.00,1.17$.

${ }^{5} \mathrm{~F}_{2}-7 \mathrm{G}_{3}\left(0_{0} 00,0.17,0.33\right), 0.83,1.00,1.17,1.33,1.50$.

${ }^{8} \mathrm{~F}_{3}{ }^{-7} \mathrm{G}_{2}(\mathbf{0 . 0 0}, 0.42,0.83), 0.42,0.83,1.25,1.67$, 2.08.

${ }^{8} \mathrm{~F}_{3}-7 \mathrm{G}_{3}(0.08,0.17,0.25), 1.00,1.08,1.17,1.25,1.33,1.42$.

${ }^{8} \mathrm{~F}_{3}{ }^{7} \mathrm{G}_{4}(0.00,0.05,0.10,0.15), 1.15,1.20,1.25,1.30,1.35,1.40,1.45$.

${ }^{8} \mathrm{~F}_{4^{-7}} \mathrm{G}_{3}(0.00,0.18,0.37,0.55), 0.80,0.98,1.17,1.35,1.53,1.72,1.90$ 。

${ }^{8} \mathrm{~F}_{4}{ }^{-7} \mathrm{G}_{4}(0.05,0.10,0.15,0.20), 1.15,1.20,1.25,1.30,1.35,1.40,1.45,1.50$.

${ }^{5} \mathrm{~F}_{4}{ }^{-7} \mathrm{G}_{5}(0.00,0.02,0.03,0.05,0.07), 1.30,1.32,1.35,1.37,1.38,1.40,1.42,1.43$ 。

${ }^{5} \mathrm{~F}_{5}-7 \mathrm{G}_{1}(0.00,0.10,0.20,0.30,0.40), 1.00,1.10,1.20,1.30,1.40,1.50,1.60,1.70,1.80$.

${ }^{5} \mathrm{~F}_{5}{ }^{-7} \mathrm{G}_{5}(0.03,0.07,0.10,0.13,0.17), 1.23,1.27,1.30,1.33, \mathbf{1 . 3 7}, \mathbf{1 . 4 0} 0,1.43,1.47,1.50,1.53$.

${ }^{8} \mathrm{~F}_{5}{ }^{7} \mathrm{G}_{6}(0.00,0.005,0.01,0.014,0.024), 1.38,1.39,1.39,1.40,1.40,1.41,1.41,1.42,1.42,1.43$.

${ }^{5} \mathrm{G}_{2}-7 \mathrm{D}_{1}^{\prime}(0.00,2.67),-2.33$, $+0.33,3.00$.

${ }^{5} \mathrm{G}_{2}-{ }^{7} \mathrm{D}_{2}^{\prime}(1.67,3.33),-1.33,+0.33,2.00,3.67$.

${ }^{5} \mathrm{G}_{2}-7 \mathrm{D}^{\prime}{ }_{3}(0.00,1.42,2.83),-1.08,+0.33,1.75,3.17,4.58$ 。

${ }^{5} \mathrm{G}_{3}-7 \mathrm{D}_{2}^{\prime}(0.00,1.08,2.17),-1.25,-0.17,+0.92,2.00,3.08$.

${ }^{8} \mathrm{G}_{3}-7 \mathrm{D}^{\prime}{ }_{3}(0.83,1.67,2.50),-0.75,+0.08,0.92,1.75,2.58,3.42$.

${ }^{8} \mathrm{G}_{3}{ }^{7} \mathrm{D}_{4}^{\prime}(0.00,0.73,1.47,2.20),-0.55,+0.18,0.92,1.65,2.38,3.12,3.85$.

${ }^{8} \mathrm{G}_{4}-7 \mathrm{D}^{\prime}{ }_{3}(0.00,0.60,1.20,1.80),-0.65,-0.05,+0.55,1.15,1.75,2.35,2.95$.

${ }^{8} \mathrm{G}_{4}-7 \mathrm{D}_{4}^{\prime}(0.50,1.00,1.50,2.00),-0.35,+0.15,0.65,1.15,1.65,2.15,2.65,3.15$.

${ }^{5} \mathrm{G}_{4}-7 \mathrm{D}_{5}^{\prime}(0.00,0.45,0.90,1.35,1.80),-0.20,+0.25,0.70,1.15,1.60,2.05,2.95,3.40$.

${ }^{8} \mathrm{G}_{5}-7 \mathrm{D}_{4}^{\prime}(0.00,0.38,0.77,1.15,1.54),-0.2 \%,+0.12,0.50,0.88,1.27,1.65,2.03,2.42,2.80$.

${ }^{5} \mathrm{G}_{5}{ }^{7} \mathrm{D}^{\prime}{ }_{5}(0.33,0.67,1.00,1.33,1.6 \%),-0.07,+0.27,0.60,0.93,1.27,1.60,1.93,2.27,2.60,2.93$.

${ }^{5} \mathrm{G}_{2}-7 \mathrm{~F}_{1}(0.00,1.17),-0.83,+0.33,1.50$.

${ }^{5} \mathrm{G}_{2}-\mathrm{F}_{2}(1.17,2.33),-0.83,+0.33,1.50,2.67$.

${ }^{5} \mathrm{G}_{2}-7 \mathrm{~F}_{3}(0.00,1.17,2.33),-0.83,+0.33,1.50,2.67,3.83$ 。

${ }^{8} \mathrm{G}_{3}-{ }^{7} \mathrm{~F}_{2}(0.00,0.58,1.17),-0.25,+0.33,0.92,1.50,2.08$.

${ }^{5} \mathrm{G}_{3}-7 \mathrm{~F}_{3}(0.58,1.17,1.75),-0.25,+0.33,0.92,1.50,2.08,2.67$.

${ }^{3} \mathrm{G}_{3}-7 \mathrm{~F}_{4}(0.00,0.58,1.17,1.75),-0.25,+0.33,0.92,1.50,2.08,2.67,3.25$ 。

${ }^{5} \mathrm{G}_{4}-7 \mathrm{~F}_{3}(0.00,0.35,0.70,1.05), 0.10,0.45,0.80,1.15,1.50,1.85,2.20$.

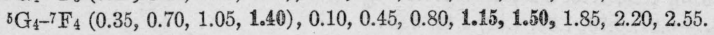

${ }^{5} \mathrm{G}_{4}-7 \mathrm{~F}_{5}(0.00,0.35,0.70,1.05,1.40), 0.10,0.45,0.80,1.15,1.50,1.85,2.20,2.55$, 2.90.

${ }^{8} \mathrm{G}_{5}-{ }^{7} \mathrm{~F}_{4}(0.00,0.23,0.47,0.70,0.93), 0.33,0.57,0.80,1.03,1.27,1.50,1.73,1.97,2.20$.

${ }^{8} \mathrm{G}_{5}-7 \mathrm{~F}_{5}(0.23,0.47,0.70,0.93,1.17), 0.33,0.57,0.80,1.03,1.27,1.50,1.73,1.97,2.20,2.43$.

${ }^{5} \mathrm{G}_{5}{ }^{-7} \mathrm{~F}_{6}(0.00,0.23,0.47,0.70,0.93,1.17), 0.33,0.57,0.80,1.03,1.27,1.50,1.73,1.97,2.20,2.43,2.67$.

${ }^{3} \mathrm{G}_{6}{ }^{7} \mathrm{~F}_{5}(0.00,0.17,0.33,0.50,0.67,0.83), 0.50,0.67,0.83,1.00,1.17,1.33,1.50,1.67,1.83,2.00,2.17$.

${ }^{5} \mathrm{G}_{6}-7 \mathrm{~F}_{6}(0.17,0.33,0.50,0.67,0.83,1.00), 0.50,0.67,0.83,1.00,1.17,1.33,1.50,1.67,1.83,2.00,2.17,2.33$.

${ }^{5} \mathrm{G}_{2}{ }^{-7} \mathrm{G}_{1}^{\prime}(0.00,0.83),-0.50,+0.33, \mathbf{1 . 1 \%}$.

${ }^{8} \mathrm{G}_{2}-{ }^{-7} \mathrm{G}^{\prime}{ }_{2}(0.50,1.00),-0.17,+0.33,0.83,1.33$.

${ }^{5} \mathrm{G}_{2}{ }^{-7} \mathrm{G}^{\prime}{ }_{3}(0.00,0.83,1.67),-0.50,+0.33,1.17,2.00$, 2.83.

${ }^{6} \mathrm{G}_{3}{ }^{-7} \mathrm{G}_{2}^{\prime}{ }_{2}(0.00,0.08,0.17), 0.75,0.83,0.92,1.00,1.08$.

${ }^{5} \mathrm{G}_{3}-7 \mathrm{G}^{\prime}{ }_{3}(0.25,0.50,0.75), 0.42,0.67,0.92,1.1 \%, 1.42,1.67$.

${ }^{8} \mathrm{G}_{3}{ }^{-7} \mathrm{G}^{\prime}{ }_{4}(0.00,0.38,0.77,1.15), 0.15,0.53,0.92,1.30,1.68,2.07$, \%.4. 15 .

${ }^{5} \mathrm{G}_{4}{ }^{-7} \mathrm{G}^{\prime}{ }_{3}(0.00,0.02,0.04,0.06), 1.09,1.11,1.13,1.15,1.17,1.19,1.21$.

${ }^{8} \mathrm{G}_{4}{ }^{7} \mathrm{G}^{\prime}{ }_{4}(0.15,0.30,0.45,0.68), 0.70,0.85,1.00,1.15,1.30,1.45,1.60,1.75$.

${ }^{5} \mathrm{G}_{4}{ }^{7} \mathrm{G}^{\prime}{ }_{5}(0.00,0.22,0.43,0.65,0.87), 0.50,0.72,0.93,1.15,1.37,1.59,1.80,2.02,2.23$.

${ }^{8} \mathrm{G}_{5}{ }^{7} \mathrm{G}^{\prime}{ }_{4}(0.09,0.03,0.07,0.10,0.13), 1.13,1.17,1.20,1.23,1.27,1.30,1.33,1.37,1.40$.

${ }^{8} \mathrm{G}_{5}{ }^{7}{ }^{-} \mathrm{G}^{\prime}{ }_{5}(0.10,0.20,0.30,0.40,0.50), 0.87,0.97,1.07,1.17,1.27,1.37,1.47,1.57,1.67,1.77$.

${ }^{5} \mathrm{G}_{5}{ }^{7}{ }^{7} \mathrm{G}_{6}{ }_{6}(0.00,0.14,0.28,0.41,0.55,0.69), 0.71,0.85,0.99,1.13,1.27,1.40,1.54,1.68,1.82,1.96,2.09$.

${ }^{5} \mathrm{G}_{6}{ }^{-7} \mathrm{G}^{\prime}{ }_{5}(0.00,0.03,0.07,0.10,0.13,0.17), 1.17,1.20,1.23,1.27,1.30,1.33,1.37,1.40,1.43,1.47,1.50$.

$\left.{ }^{5} \mathrm{G}_{6}{ }^{7} \mathrm{G}^{\prime}{ }_{6}(0.07,0.14,0.21,0.29,0.36,0.43), 0.98,1.05,1.12,1.19,1.26,1.33,1.41,1.48,1.55,1.62,1.69,1.76.\right]$

${ }^{5} \mathrm{G}_{6}{ }^{7} \mathrm{G}^{\prime}{ }_{7}(0.00,0.10,0.19,0.29,0.38,0.48,0.57), 0.86,0.95,1.05,1.14,1.24,1.33,1.43,1.52,1.62,1.72,1.81,1.91$, 2.00. 
TABLE 14.-Landé $g$ values with decimal equivalents for ierms of even multiplicity

\begin{tabular}{|c|c|c|c|c|}
\hline$-\frac{4}{3}=-1.333$ & ${ }^{8} \mathrm{G}_{1}$. & $\frac{22}{17}=$ & 1. 294 & ${ }^{8} \mathrm{I}_{8}$ \\
\hline$-\frac{2}{3}=-0.667$ & ${ }^{6} \mathrm{~F}_{1}$. & $\frac{186}{1183}=$ & 1. 301 & ${ }^{8} \mathrm{H}_{6}$. \\
\hline$-\frac{2}{5}=-0.400$ & ${ }^{8} \mathrm{H}_{2}$. & $\frac{46}{38}=$ & 1.314 & ${ }^{6} \mathrm{~F}_{3}$ \\
\hline$\frac{0}{8}=0.000$ & ${ }^{4} \mathrm{D}_{1},{ }^{6} \mathrm{G}_{2},{ }^{8} \mathrm{I}_{3}$. & $\frac{4}{3}=$ & 1.333 & ${ }^{2} \mathrm{P}_{2},{ }^{4} \mathrm{~F}_{5},{ }^{6} \mathrm{H}_{8}$. \\
\hline$\frac{2}{7}=0.286$ & ${ }^{6} \mathrm{H}_{3}$. & $\frac{43}{32} \frac{2}{3}=$ & 1. 337 & ${ }^{8} I_{9}$ \\
\hline$\frac{2}{6}=0.400$ & ${ }^{4} \mathrm{~F}_{2}$ & & & ${ }^{6} \mathrm{G}_{6}$. \\
\hline$\frac{4}{8}=0.444$ & $6 \mathrm{I}_{4}$ & $\begin{array}{l}\frac{88}{85}= \\
\frac{86}{86}=\end{array}$ & $\begin{array}{l}1.354 \\
1.365\end{array}$ & $\begin{array}{l}{ }^{8} \mathrm{H}_{7} \\
{ }^{8} \mathrm{G}_{4}\end{array}$ \\
\hline$\frac{4}{7}=0.571$ & ${ }^{4} \mathrm{G}_{3}$. & $\begin{array}{l}63= \\
\frac{25}{19}=\end{array}$ & $\begin{array}{l}1.000 \\
1.368\end{array}$ & $8 I_{10}$ \\
\hline $\begin{aligned} \frac{2}{3} & =0.667 \\
34 & =0.686\end{aligned}$ & ${ }^{2} \mathrm{P}_{1},{ }^{4} \mathbf{H}_{4},{ }^{8} \mathbf{I}_{4}$ & $\frac{48}{36}=$ & 1. 371 & ${ }^{4} \mathrm{D}_{3}$. \\
\hline & & $\frac{18}{13}=$ & 1. 385 & ${ }^{6} \mathrm{G}_{7}$. \\
\hline$\frac{8}{11}=0.727$ & ${ }^{4} \mathrm{I}_{5}$. & $\frac{118}{\frac{118}{5}}=$ & 1. 388 & ${ }^{8} \mathrm{H}_{8}$. \\
\hline$\frac{4}{6}=0.800$ & ${ }^{2} \mathrm{D}_{2}$ & $\frac{8}{68}=$ & 1. 397 & ${ }^{6} \mathrm{~F}_{4}$ \\
\hline$\frac{85}{63}=0.825$ & ${ }^{6} \mathrm{H}_{4}$. & $\begin{array}{l}\frac{63}{456} \\
\frac{456}{325}=\end{array}$ & 1.412 & ${ }^{8} \mathrm{H}_{9}$. \\
\hline$\frac{7}{11}=0.828$ & & $\begin{array}{l}325 \\
\frac{140}{99}=\end{array}$ & 1.414 & ${ }^{8} G_{5}$ \\
\hline$\frac{6}{7}=0.857$ & ${ }^{2} \mathrm{~F}_{3},{ }^{6} \mathrm{G}_{3}$ & $\frac{10}{7}=$ & 1. 429 & ${ }^{4} \mathrm{D}_{4}$. \\
\hline$\frac{8}{8}=0.889$ & ${ }^{2} \mathrm{G}_{4}$ & $\frac{14 \frac{2}{9}}{9}=$ & 1. 434 & ${ }^{6} \mathrm{~F}_{5}$ \\
\hline$\frac{10}{11}=0.909$ & ${ }^{2} \mathrm{H}_{5}$. & $\frac{298}{1106}=$ & 1. 441 & ${ }^{8} \mathrm{G}_{6}$. \\
\hline$\frac{12}{13}=0.923$ & ${ }^{2} \mathrm{I}_{6}$ & $\frac{18}{112}=$ & 1.455 & ${ }^{6} \mathrm{~F}_{6}$ \\
\hline$\frac{14}{18}=0.933$ & ${ }^{8} \mathrm{G}_{2}$ & $\frac{28 \hat{1}}{118}=$ & 1.456 & ${ }^{8} \mathrm{G}_{7}$. \\
\hline$\frac{138}{14}=0.965$ & ${ }^{4} \mathrm{I}_{6}$ & $\frac{2}{15}=$ & 1. 467 & ${ }^{8} \mathrm{G}_{8}$. \\
\hline$\frac{32}{33}=0.970$ & ${ }^{4} \mathrm{H}_{5},{ }^{8} \mathrm{I}_{5}$. & $\begin{array}{l}\frac{15}{15}= \\
\frac{13}{13}=\end{array}$ & 1. 538 & ${ }^{8} \mathrm{~F}_{7}$ \\
\hline$\frac{6}{6} \frac{3}{6}=0.984$ & ${ }^{4} \mathrm{G}_{4}$ & $\frac{23}{1} \frac{2}{3}=$ & 1. 552 & ${ }^{8} \mathrm{~F}_{6}$ \\
\hline$\frac{38}{36}=1.029$ & ${ }^{4} \mathrm{~F}_{3}$. & $\frac{13}{8}=$ & 1.556 & ${ }^{6} \mathrm{D}_{5}$ \\
\hline$\frac{148}{143}=1.035$ & ${ }^{6} \mathrm{I}_{6}$ & $\frac{5}{3} \frac{2}{3}=$ & 1.576 & ${ }^{8} \mathrm{~F}_{5}$. \\
\hline$\frac{22}{21}=1.048$ & ${ }^{8} \mathrm{H}_{4}$. & $\frac{100}{83}=$ & 1. 587 & ${ }^{6} \mathrm{D}_{4}$. \\
\hline$\frac{16}{16}=1.067$ & ${ }^{6} \mathrm{~F}_{2}$ & $\frac{8}{6}=$ & 1.600 & ${ }^{4} \mathrm{P}_{3}$ \\
\hline$\frac{106}{90}=1.071$ & ${ }^{6} \mathrm{H}_{5}$. & $\frac{34}{21}=$ & 1. 619 & ${ }^{8} \mathrm{~F}_{4}$ \\
\hline$\frac{14}{13}=1.077$ & ${ }^{2} \mathrm{I}_{\overline{7}}$. & $\frac{18}{11}=$ & 1. 636 & ${ }^{8} \mathrm{D}_{6}$ \\
\hline$\frac{12}{11}=1.091$ & ${ }^{2} \mathrm{H}_{6}$. & $\frac{88}{38}=$ & 1. 657 & ${ }^{6} \mathrm{D}_{3}$. \\
\hline$\frac{z z}{6}=1.108$ & ${ }^{4} \mathrm{I}_{7}$. & $\frac{5}{3} \frac{5}{3}=$ & 1.697 & ${ }^{8} \mathrm{D}_{5}$. \\
\hline$\frac{10}{6}=1.111$ & ${ }^{2} \mathrm{G}_{5}$. & $\frac{13}{17}=$ & 1. 714 & ${ }^{6} \mathrm{P}_{4},{ }^{8} \mathrm{~F}_{3}$. \\
\hline$\frac{16 \frac{2}{16}}{14}=1.133$ & ${ }^{4} \mathrm{H}_{6},{ }^{8} \mathrm{I}_{6}$. & $\frac{26}{15}=$ & 1. 733 & ${ }^{4} \mathrm{P}_{2}$ \\
\hline$\frac{8}{7}=1.143$ & ${ }^{2} \mathrm{~F}_{4},{ }^{6} \mathrm{G}_{4}$. & $\frac{16}{8}=$ & 1. 778 & ${ }^{8} \mathrm{P}_{5}$ \\
\hline$\frac{228}{105}=1.159$ & ${ }^{6} \mathbf{I}_{7}$. & $\frac{38}{21}=$ & 1.809 & ${ }^{8} \mathrm{D}_{4}$. \\
\hline$\frac{118}{18}=1.172$ & ${ }^{4} \mathrm{G}_{5}$. & $\begin{array}{l}\frac{28}{15}= \\
\frac{21}{15}=\end{array}$ & 1.867 & ${ }^{6} \mathrm{D}_{2}$. \\
\hline $6=1.200$ & ${ }^{2} \mathrm{D}_{3},{ }^{4} \mathrm{D}_{2},{ }^{4} \mathrm{I}_{8}$ & $\frac{66}{38}=$ & 1.886 & ${ }^{6} \mathrm{P}_{3}$ \\
\hline$\frac{17}{17 \frac{3}{3}}=1.203$ & ${ }^{6} \mathrm{H}_{6}$. & $\frac{12 \frac{2}{6}}{6}=$ & 1. 937 & ${ }^{8} \mathrm{P}_{4}$ \\
\hline$\frac{40}{63}=1.212$ & ${ }^{8} \mathrm{H}_{5}$. & $2=$ & & ${ }^{2} \mathrm{~S}_{1},{ }^{4} \mathrm{~S}_{2},{ }^{6} \mathrm{~S}_{2},{ }^{8} \mathrm{~S}_{4}, 8 \mathrm{~F}_{2}$ \\
\hline$\frac{16}{13}=1.231$ & ${ }^{4} \mathrm{H}_{7},{ }^{8} \mathrm{I}_{7}$. & $\frac{7}{\frac{7}{5}}=$ & 2. 057 & ${ }^{8} \mathrm{D}_{3}$. \\
\hline$\frac{78}{63}=1.238$ & ${ }^{4} \mathrm{~F}_{4}$ & 列 & 2. 286 & ${ }^{8} \mathrm{P}_{3}$ \\
\hline$\frac{316}{268}=1.239$ & ${ }^{6} I_{8}$. & $\frac{12}{6}=$ & 2. 400 & ${ }^{6} \mathrm{P}_{2}$ \\
\hline$\frac{4}{3} \frac{4}{5}=1.257$ & ${ }^{8} \mathrm{G}_{3}$ & $\frac{s}{3}=$ & 2. 667 & ${ }^{4} \mathrm{P}_{1}$ \\
\hline$\frac{16}{13}=1.273$ & ${ }^{4} \mathrm{G}_{6},{ }^{6} \mathrm{G}_{5}$. & $\frac{3}{\frac{3}{6}}=$ & 2. 800 & ${ }^{8} \mathrm{D}_{2}$. \\
\hline$\frac{50}{38}=1.282$ & ${ }^{6} \mathrm{H}_{7}$ & $\frac{6}{3 \frac{0}{3}}=$ & 3. 333 & ${ }^{6} \mathrm{D}_{1}$ \\
\hline$\frac{41 \frac{8}{3}}{32}=1.294$ & ${ }^{6} \mathrm{I}_{9}$. & $4=$ & 4. 000 & ${ }^{8} \mathrm{~F}_{1}$. \\
\hline
\end{tabular}


TABLE 15.-Landé g values with decimal equivalents for terms of odd multiplicity

\begin{tabular}{|c|c|c|c|c|}
\hline$-\frac{1}{2}=-0.500$ & ${ }^{7} \mathrm{G}_{1}$. & $\frac{17}{14}=$ & 1. 214 & ${ }^{5} \mathrm{H}_{6}$. \\
\hline$\frac{0}{0}=$ & ${ }^{1} \mathrm{~S}_{0},{ }^{3} \mathrm{P}_{0},{ }^{8} \mathrm{D}_{0},{ }^{7} \mathrm{~F}_{0}$ & $\begin{array}{ll}\frac{50}{56}= \\
56\end{array}$ & 1. 232 & \\
\hline$\frac{0}{2}, \frac{0}{6}=0.000$ & ${ }^{5} \mathrm{~F}_{1},{ }^{7} \mathrm{H}_{2}$ & $\frac{5}{4}=$ & 1. 250 & ${ }^{3} \mathrm{~F}_{4}, 5 \mathrm{~F}_{3},{ }^{8} \mathrm{I}_{8}$ \\
\hline$\frac{1}{4}=0.250$ & $\mathrm{I}_{3}$ & $1 \frac{19}{15}=$ & 1. 267 & \\
\hline$\frac{1}{3}=0.333$ & ${ }^{5} \mathrm{G}_{2}$. & $\frac{9}{7}=$ & 1. 286 & ${ }^{5} \mathrm{H}_{7},{ }^{7} \mathrm{H}_{6}$. \\
\hline$\frac{1}{2}=0.500$ & ${ }^{3} \mathrm{D}_{1},{ }^{5} \mathrm{H}_{3}$. & $\begin{array}{l}\frac{21}{24}= \\
13=\end{array}$ & 1. 292 & ${ }^{7} \mathrm{I}_{8}$ \\
\hline$\frac{3}{6}=0.600$ & ${ }^{5} I_{4}$. & $\begin{aligned} \frac{1}{10} & = \\
\frac{4}{3} & =\end{aligned}$ & $\begin{array}{l}1.300 \\
1.333\end{array}$ & ${ }^{3} \mathrm{D}_{3},{ }^{5} \mathrm{G}_{6}, \mathrm{~T}_{9}$ \\
\hline$\frac{2}{3}=0.667$ & ${ }^{3} \mathrm{~F}_{2}$ & $\frac{27}{20}=$ & 1.350 & ${ }^{5} \mathrm{~F}_{4}$ \\
\hline$\frac{3}{4}=0.750$ & ${ }^{3} \mathrm{G}_{3},{ }^{7} \mathrm{H}_{3}, 7^{7} \mathrm{I}_{4}$ & $\frac{75}{656}=$ & 1.339 & ${ }^{7} \mathrm{H}_{7}$. \\
\hline$\frac{4}{6}=0.800$ & & $\frac{41}{30}=$ & 1. 367 & ${ }^{7} \mathrm{G}_{5}$. \\
\hline$\frac{s}{6}=0.833$ & ${ }^{3} \mathrm{I}_{5},{ }^{7} \mathrm{G}_{2}$ & $\begin{array}{l}30 \\
\frac{11}{8}=\end{array}=$ & 1.375 & ${ }^{7} \mathrm{H}_{8}$ \\
\hline$\frac{\circ}{10}=0.900$ & ${ }^{5} \mathrm{H}_{4},{ }^{5} \mathrm{I}_{5}$. & $\frac{\tau}{6}=$ & 1.400 & ${ }^{5} \mathrm{~F}_{5}$. \\
\hline$\frac{11}{12}=0.917$ & ${ }^{5} \mathrm{G}_{3}$. & $\frac{59}{42}=$ & 1.405 & ${ }^{7} \mathrm{G}_{6}$. \\
\hline $1=1.000$ & ${ }^{1} \mathrm{P}_{1},{ }^{1} \mathrm{D}_{2},{ }^{1} \mathrm{~F}_{3},{ }^{1} \mathrm{G}_{4},{ }^{1} \mathrm{H}_{5},{ }^{1} \mathrm{I}_{6},{ }^{5} \mathrm{~F}_{2},{ }^{7 \mathrm{I}_{5}}$ & ${ }^{10}=$ & 1.429 & ${ }^{7} \mathrm{G}_{7}$. \\
\hline$\frac{43}{42}=1.024$ & & $\frac{3}{2}=$ & 1. 500 & ${ }^{3} \mathrm{P}_{1},{ }^{3} \mathrm{P}_{2},{ }^{7} \mathrm{D}_{1},{ }^{7} \mathrm{D}_{2},{ }^{7} \mathrm{D}_{3},{ }^{7} \mathrm{D}_{4},{ }^{7} \mathrm{~F}_{1}$, \\
\hline $\begin{array}{l}\frac{3}{3} \frac{1}{6}=1.033 \\
\frac{21}{20}=1.050\end{array}$ & $\begin{array}{l}{ }^{3} \mathrm{H}_{5} . \\
{ }^{3} \mathrm{G}_{4},{ }^{7} \mathrm{H}_{4} .\end{array}$ & $\begin{aligned} \frac{8}{5} & = \\
\frac{3}{30} & =\end{aligned}$ & $\begin{array}{l}1.600 \\
1.650\end{array}$ & $\begin{array}{l}{ }^{7} \mathrm{D}_{5} \mathrm{D}_{2},{ }^{7} \mathrm{~T}_{3} \\
7 \mathrm{D}_{4} .\end{array}$ \\
\hline$\frac{16}{14}=1.071$ & & 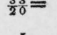 & & \\
\hline$\frac{13}{12}=1.083$ & ${ }^{3} \mathrm{~F}_{3}$. & $\frac{x^{3}}{3}=$ & 1. 667 & ${ }^{5} \mathrm{P}_{3 .}$ \\
\hline$\frac{11}{10}=1.100$ & ${ }^{6} \mathrm{H}_{5}$. & $\begin{array}{l}\frac{7}{4}= \\
\frac{11}{8}=\end{array}$ & $\begin{array}{l}1.750 \\
1.833\end{array}$ & $\begin{array}{l}{ }^{7} \mathrm{P}_{4},{ }^{7} \mathrm{D}_{3} \\
{ }^{5} \mathrm{P}_{2}\end{array}$ \\
\hline$\frac{8}{7}=1.143$ & ${ }^{3} I_{7}, I_{6}$. & $\frac{28}{12}=$ & 1. 917 & ${ }^{7} \mathrm{P}_{3}$ \\
\hline$\frac{23}{20}=1.150$ & ${ }^{6} \mathrm{G}_{4}$ & $2=$ & 2.000 & ${ }^{3} \mathrm{~S}_{1},{ }^{5} \mathrm{~S}_{2},{ }^{7} \mathrm{~S}_{3},{ }^{7} \mathrm{D}_{2}$ \\
\hline $\begin{aligned} \frac{5}{8} & =1.167 \\
33 & =1.179\end{aligned}$ & ${ }^{3} \mathrm{D}_{2},{ }^{3} \mathrm{H}_{6},{ }^{7} \mathrm{G}_{3}$. & $\frac{7}{3}=$ & 2. 333 & ${ }^{7} \mathrm{P}_{2}$ \\
\hline$\frac{3}{23}=1.179$ & $\begin{array}{l}{ }^{3} I_{7} . \\
{ }^{3} \mathrm{G}_{5},{ }^{7} \mathrm{H}_{5} .\end{array}$ & $\begin{array}{l}\frac{5}{2}= \\
3=\end{array}$ & 2. 500 & ${ }^{5} \mathrm{P}_{1}$ \\
\hline & & $3=$ & 3.000 & ${ }^{7} \mathrm{D}_{1}$ \\
\hline
\end{tabular}

WASHington, June 22, 1928. 


\title{
The Bureau of Standards Journal of Research
}

\author{
CONTENTS OF PREVIOUS ISSUES
}

\section{July, 1928 (Vol. 1, No. 1)}

RP1. Accelerated tests of organic protective coatings. Percy H. Walker and E. F. Hickson.

RP2. Measurement of the tread movement of pneumatic tires and a discussion of the probable relation to tread wear. W. L. Holt and C. M. Cook.

RP3. Absolute methods in reflectometry. H. J. McNicholas.

RP4. Interferometer measurements of wave lengths in the vacuum arc spectra of titanium and other elements. C. C. Kiess.

RP5. Analysis of bauxite and of refractories of high alumina content. G. E. F. Lundell and J. I. Hoffman.

\section{August, 1928 (Vol. 1, No. 2)}

RP6. Some measurements of the transmission of ultra-violet radiation through various kinds of fabrics. W. W. Coblentz, R. Stair, and C. W. Schoffstall.

RP7. Tinting strength of pigments. H. D. Bruce.

RP8. Wave-length measurements in the arc and spark spectra of hafnium. William F. Meggers.

RP9. Tests of the effect of brackets in reinforced concrete rigid frames. Frank E. Richart.

RP10. Accelerated laboratory corrosion test methods for zinc-coated steel. Edward C. Groesbeck and William A. Tucker.

\section{September, 1928 (Vol. 1, No. 3)}

RP11. Standard solar wave lengths (3592-7148A). Keivin Burns, William F. Meggers, and C. C. Kiess.

RP12. Wave lengths and zeeman effects in yttrium spectra. William F. Meggers.

RP13. Wear and mechanical properties of railroad bearing bronzes at different temperatures. H. J. French, S. J. Rosenberg, W. LeC. Harbaugh, and H. C. Cross.

RP14. Steel for casehardening-normal and abnormal steel. S. Epstein and H. S. Rawdon.

RP15. Strain markings in mild steel under tension. H. S. Rawdon.

These will be available as separates for purchase from the Superintendent of Documents, United States Government Printing Office, Washington, D. C.

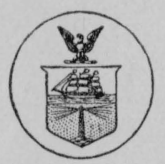

